Ying Ma, Sheng Chen, Fellow, IEEE, Chengwen Xing, Member, IEEE, Xiangyuan Bu, and Lajos Hanzo, Fellow, IEEE

Abstract-Distributed radar systems are capable of enhancing the detection performance by using multiple widely spaced distributed antennas. With prior statistic information of targets, resource allocation is of critical importance for further improving the system's achievable performance. In this paper, the total transmitted power is minimized at a given mean-square target-estimation error. We derive two iterative decomposition algorithms for solving this nonconvex constrained optimization problem, namely, the optimality condition decomposition (OCD)-based and the alternating direction method of multipliers (ADMM)-based algorithms. Both the convergence performance and the computational complexity of our algorithms are analyzed theoretically, which are then confirmed by our simulation results. The OCD method imposes a much lower computational burden per iteration, while the ADMM method exhibits a higher per-iteration complexity, but as a benefit of its significantly faster convergence speed, it requires less iterations. Therefore, the ADMM imposes a lower total complexity than the OCD. The results also show that both of our schemes outperform the state-of-the-art benchmark scheme for the multiple-target case, in terms of the total power allocated, at the cost of some degradation in localization accuracy. For the single-target case, all the three algorithms achieve similar performance. Our ADMM algorithm has similar total computational complexity per iteration and convergence speed to those of the benchmark.
\end{abstract}

Index Terms-Alternating direction method of multipliers, localization, multiple-input multiple-output radar, optimality condition decomposition, resource allocation.

\section{INTRODUCTION}

$\mathbf{M}$ ULTIPLE-input multi-output (MIMO) radar systems relying on widely-separated antennas have attracted considerable attention from both industry and academia. The family of distributed MIMO radar systems is capable of significantly improving the estimation/detection performance [1]-[6] by exploiting the increased degrees of freedom resulting from the improved spatial diversity. In particular, distributed radar systems are capable of improving accuracy of target location and

Manuscript received March 8, 2016; revised June 17, 2016 and July 18, 2016; accepted August 9, 2016. The associate editor coordinating the review of this manuscript and approving it for publication was Dr. Fauzia Ahmad. This work was supported in part by the National Natural Science Foundation of China under Grants 61421001 and 61671058.

Y. Ma, C. Xing, and X. Bu are with the School of Information and Electronics, Beijing Institute of Technology, Beijing 100081, China (e-mail: mayingbit2011@gmail.com; xingchengwen@gmail.com; bxy@bit.edu.cn).

$\mathrm{S}$. Chen is with the School of Electronics and Computer Science, University of Southampton, Southampton SO17 1BJ, U.K., and also with King Abdulaziz University, Jeddah 21589, Saudi Arabia (e-mail: sqc@ecs.soton.ac.uk).

L. Hanzo is with the School of Electronics and Computer Science, University of Southampton, Southampton SO17 1BJ, U.K. (e-mail: 1h@ecs.soton.ac.uk).

Color versions of one or more of the figures in this paper are available online at http://ieeexplore.ieee.org.

Digital Object Identifier 10.1109/TSP.2016.2602801

\title{
Decomposition Optimization Algorithms for Distributed Radar Systems
}

velocity estimation by exploiting the different Doppler estimates from multiple spatial directions [7]-[10].

Naturally, the localization performance of MIMO radar systems relying on widely-spaced distributed antennas, quantified in terms of the mean square estimation error (MSE), is determined by diverse factors, including effective signal bandwidth, the signal-to-noise ratio (SNR), the product of the numbers of transmit and receive antennas, etc [11]. Since the SNR is influenced by the path loss, the target radar cross section (RCS) and the transmitted power, the attainable localization performance can be improved by increasing either the number of participating radars or the transmitted power. However, simply increasing the amount of resources without considering the cooperation among the individual terminals is usually far from optimal.

In most traditional designs, the system's power budget is usually allocated to the transmit radars and it is fixed [6], [10], which is easy to implement and results in the simplest network structure. However, when prior estimation of the target RCS is available, according to estimation theory, uniform power allocation is far from the best strategy. In battlefields, a radar system is usually supported by power-supply trucks, but under hostile environments, their number is strictly limited. Thus, how to allocate limited resources to multiple radar stations is of great importance for maximizing the achievable performance. In other words, power allocation substantially affects the detection performance of multi-radar systems.

Recently, various studies used the Cramer-Rao lower bound (CRLB) for evaluating the performance of MIMO radar systems [11]-[16]. A power allocation scheme [12] based on CRLB was designed for multiple radar systems with a single target. The resultant nonconvex optimization problem was solved by relaxation and a domain-decomposition method. Specifically, in [12] the total transmitted power was minimized at a given estimation MSE threshold. However the algorithm of [12] was not designed for multiple-target scenarios, which are often encountered in practice. In [13] a power allocation algorithm was proposed for the multiple-target case, which is equally applicable to the single-target senario.

Against this background, in this paper, we propose two iterative decomposition methods, which are referred to as the optimality condition decomposition (OCD) [17] and the alternating direction method of multipliers (ADMM) [18] algorithms, in order to minimize the total transmitted power while satisfying a predefined estimation MSE threshold. These two algorithms can be applied to both multiple-target and single-target scenarios. The ADMM method has been widely adopted for solving convex problems. In this paper, we extend the ADMM algorithm to nonconvex problems and show that it is capable of converging. 
It is worth pointing out that Simonetto and Leus [19] applied the ADMM method to solve a localization problem in a sensor network by converting the nonconvex problem to a convex one using rank-relaxation. However, the algorithm of [19] cannot be applied to our problem, because the task of [19] is that of locating sensors, which is not directly related to the signal waveform and power. Furthermore, the maximum likelihood (ML) criterion can be used for solving this sensor localization problem. However, our task is to assign the power of every MIMO radar transmitter, and at the time of writing it is an open challenge to design the ML estimator for this task [11]. The main contributions of our work are as follows.

- We propose two iterative decomposition algorithms, namely, the OCD-based and ADMM-based methods, for both multiple-target and single-target scenarios. The convergence of these two algorithms is analyzed theoretically and verified by simulations. Both these two methods are capable of converging to locally optimal solutions. The complexity analysis of the two algorithms is provided and it is shown that the OCD method imposes a much lower computational burden per iteration, while the ADMM method enjoys a significantly faster convergence speed and therefore it actually imposes a lower total complexity.

- In the multiple-target case, we demonstrate that both of our two algorithms outperform the state-of-the-art benchmark scheme of [13], in terms of the total power allocated at the expense of some degradation in localization accuracy. We show furthermore that our ADMM-based algorithm and the algorithm of [13] have similar convergence speed and total computational complexity.

- In the single-target case, we show that all the three methods attain a similar performance, since the underlying optimization problems are identical. We also prove that the closed-form solution of [12] is invalid for the systems with more than three transmit radars and we propose a beneficial suboptimal closed-form solution.

The paper is organized as follows. In Section II, the MIMO radar system model is introduced and the corresponding optimization problem is formulated. Our power allocation strategies are proposed in Section III for both the multiple-target and single-target cases, while our convergence and complexity analysis is provided in Section IV. Section V presents our simulation results for characterizing the attainable performance of the proposed algorithms which are then compared to the scheme of [13]. Finally, our conclusions are offered in Section VI.

Throughout our discussions, the following notational conventions are used. Boldface lower- and upper-case letters denote vectors and matrices, respectively. The transpose, conjugate and inverse operators are denoted by $(\cdot)^{\mathrm{T}},(\cdot)^{*}$ and $(\cdot)^{-1}$, respectively, while $\operatorname{Tr}(\cdot)$ stands for the matrix trace operation and $\operatorname{diag}\left(x_{1}, x_{2}, \cdots, x_{n}\right)$ or $\operatorname{diag}(\mathbf{x})$ is the diagonal matrix with the specified diagonal elements. Additionally, $\operatorname{diag}\left(\mathbf{X}_{1}, \cdots, \mathbf{X}_{K}\right)$ and $\operatorname{diag}\left(\mathbf{x}_{1}, \cdots, \mathbf{x}_{K}\right)$ denotes the block diagonal matrices with the specified sub-matrices and vectors, respectively, at the corresponding block diagonal positions. The operator $\mathrm{v}_{\text {diag }}(\mathbf{X})$ forms a vector using the diagonal elements of square matrix $\mathbf{X}$, while $\mathrm{E}\{\cdot\}$ denotes the expectation operator and $\otimes$ is the

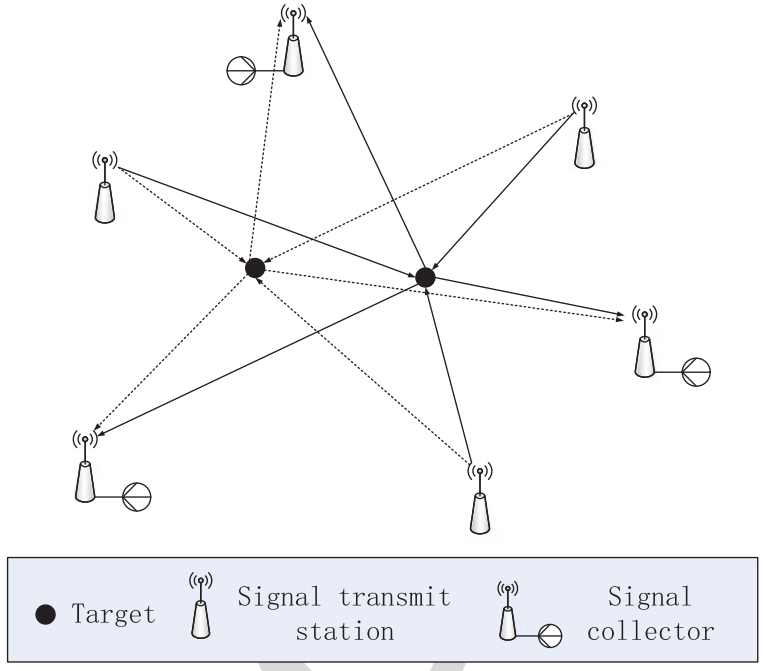

Fig. 1. Illustration of distributed radar network.

Kronecker product operator. The sub-matrix consisting of the 145 elements of the $i_{1}$ to $i_{2}$ rows and $j_{1}$ to $j_{2}$ columns of $\mathbf{A}$ is 146 denoted by $[\mathbf{A}]_{\left[i_{1}: i_{2} ; j_{1}: j_{2}\right]}$, and the $i$ th row and $j$ th column ele- 147 ment of $\mathbf{A}$ is given by $[\mathbf{A}]_{i, j}$. Similarly, $[\mathbf{a}]_{\left[i_{1}: i_{2}\right]}$ is the vector 148 consisting of $i_{1}$ th to $i_{2}$ th elements of $\mathbf{a}$. The magnitude operator 149 is given by $|\cdot|$, and $\|\cdot\|$ denotes the vector two-norm or matrix 150 Frobenius norm. $\mathbf{I}_{K}$ is the identity matrix of size $K \times K$ and $\mathbf{0} 151$ is the zero matrix/vector of an appropriate size, while $\mathbf{1}$ denotes 152 the vector of an appropriate size, whose elements are all equal 153 to one. Finally, $\Re[]$ denotes the real part of a complex value and 154 $\mathrm{j}=\sqrt{-1}$ represents the imaginary axis.

\section{SYSTEM MODEL}

The MIMO radar system consists of $M$ transmit radars and $N$ receive radars which cooperate to locate $K$ targets, as illustrated in Fig. 1. The $M$ transmit radars are positioned at the coordinates $\left(x_{m}^{t x}, y_{m}^{t x}\right)$ for $1 \leq m \leq M$, and the $N$ receive radars are positioned at $\left(x_{n}^{r x}, y_{n}^{r x}\right)$ for $1 \leq n \leq N$, while the position of target $k$ is $\left(x_{k}, y_{k}\right)$. A set of mutually orthogonal waveforms are transmitted from the transmit radars, and the corresponding baseband signals are denoted by $\left\{s_{m}(t)\right\}_{m=1}^{M}$ with normalized power, i.e., $\int_{\tau_{m}}\left|s_{m}(t)\right|^{2} d t=1$, where $\tau_{m}$ is the duration of the $m$ th transmitted signal. Furthermore, the orthogonality of the transmitted waveforms can always be guaranteed even for different time delays, i.e., $\int_{\tau_{m}} s_{m}(t) s_{m^{\prime}}^{*}(t-\tau) d t=0$ for $m^{\prime} \neq m$. The narrowband signals of the transmitted waveforms have the effective bandwidth $\beta_{m}$ specified by

$$
\beta_{m}^{2}=\frac{\int_{W} f^{2}\left|S_{m}(f)\right|^{2} d f}{\int_{W}\left|S_{m}(f)\right|^{2} d f}(\mathrm{~Hz})^{2}
$$

where $W$ is the frequency range of the signals, and $S_{m}(f)$ is the Fourier transform of $s_{m}(t)$ transmitted from the $m$ th transmit radar. The transmitted powers of the different antennas, denoted by $\mathbf{p}=\left[p_{1} p_{2} \cdots p_{M}\right]^{\mathrm{T}}$, are constrained by their corresponding 
minimum and maximum values specified by

$$
\begin{aligned}
& \mathbf{p}_{\min }=\left[p_{1_{\text {min }}} p_{2_{\text {min }}} \cdots p_{M_{\text {min }}}\right]^{\mathrm{T}}, \\
& \mathbf{p}_{\max }=\left[p_{1_{\max }} p_{2_{\max }} \cdots p_{M_{\max }}\right]^{\mathrm{T}} .
\end{aligned}
$$

176

202 203 204
The upper bound $p_{m_{\max }}$ is determined by the design and the lower bound $p_{m_{\min }}$ is used to guarantee that the transmit radar $m$ operates at an appropriate SNR. Let the propagation path spanning from the transmitter $m$ to the target $k$ and from the target $k$ to the receiver $n$ be defined as the channel $(m, k, n)$. Then the propagation time $\tau_{m, n}^{(k}$ of the channel $(m, k, n)$ can be calculated by $\tau_{m, n}^{(k}=\left(R_{m, k}^{t x}+R_{n, k}^{r x}\right) / c$, where $c$ is the speed of light, $R_{m, k}^{t x}=\sqrt{\left(x_{m}^{t x}-x_{k}\right)^{2}+\left(y_{m}^{t x}-y_{k}\right)^{2}}$ is the distance from transmitter $m$ to target $k$, and $R_{n, k}^{r x}=$ $\sqrt{\left(x_{n}^{r x}-x_{k}\right)^{2}+\left(y_{n}^{r x}-y_{k}\right)^{2}}$ is the distance from target $k$ to receiver $n$. The time delay $\tau_{m, n}^{(k}$ is used to estimate the position of targets. For far field signals, by retaining only the linear terms of its Taylor expansion, $\tau_{m, n}^{(k}$ can be approximated as a linear function of $x_{k}$ and $y_{k}$

$$
\begin{aligned}
\tau_{m, n}^{(k} \simeq & -\frac{x_{k}}{c}\left(\cos \theta_{m}^{(k}+\cos \varphi_{n}^{(k)}\right) \\
& -\frac{y_{k}}{c}\left(\sin \theta_{m}^{(k}+\sin \varphi_{n}^{(k}\right),
\end{aligned}
$$

where $\theta_{m}^{(k}$ is the bearing angle of the transmitting radar $m$ to the target $k$ and $\varphi_{n}^{(k}$ is the bearing angle of the receiving radar $n$ to the target $k$, both measured with respect to the $x$ axis.

Let the complex-valued reflectivity coefficient $h_{m, n}^{(k}$ represent the attenuation and phase rotation of channel $(m, k, n)$. The baseband signal at receive radar $n$ can be expressed as

$$
r_{n}(t)=\sum_{k=1}^{K} \sum_{m=1}^{M} \sqrt{p_{m}} h_{m, n}^{(k} s_{m}\left(t-\tau_{m, n}^{(k}\right)+\omega_{n}(t),
$$

where $\omega_{n}(t)$ is a circularly symmetric complex Gaussian white noise, which is bandlimited to the system bandwidth $W$ and hence has a zero mean and $E\left\{\left|\omega_{n}(t)\right|^{2}\right\}=\sigma^{2}$. In our work, the path-loss $\kappa_{m, n}^{k}$ is chosen as

$$
\kappa_{m, n}^{(k} \propto \frac{1}{\left(R_{m, k}^{t x}\right)^{2}\left(R_{n, k}^{r x}\right)^{2}}
$$

Thus, given the complex target $\operatorname{RCS} \zeta_{m, n}^{(k}$, the channel coefficient $h_{m, n}^{(k}$ is given by

$$
h_{m, n}^{(k}=\zeta_{m, n}^{(k} \sqrt{\kappa_{m, n}^{(k}}=h_{m, n}^{(k, \operatorname{Re}}+\mathbf{j} h_{m, n}^{(k, \operatorname{Im}},
$$

02 where $h_{m, n}^{(k, \mathrm{Re}}$ and $h_{m, n}^{(k, \text { Im }}$ are the real and imaginary parts of $h_{m, n}^{(k}$. Let us collect all the channel coefficients associated with the target $k$ in the $(2 M N \times 1)$-element real-valued vector as $\mathbf{h}_{k}=\left[h_{1,1}^{(k, \operatorname{Re}} \cdots h_{1, N}^{(k, \operatorname{Re}} \cdots h_{M, N}^{(k, \operatorname{Re}} h_{1,1}^{(k, \operatorname{Im}} \cdots h_{1, N}^{(k, \operatorname{Im}} \cdots h_{M, N}^{(k, \operatorname{Im}}\right]^{\mathrm{T}}$.
Similarly, we introduce the $(N M \times 1)$-element real vectors

$$
\begin{aligned}
\left|\mathbf{h}^{(k}\right|^{2} & =\left[\left|h_{1,1}^{(k}\right|^{2} \cdots\left|h_{1, N}^{(k}\right|^{2} \cdots\left|h_{M, 1}^{(k}\right|^{2} \cdots\left|h_{M, N}^{(k}\right|^{2}\right]^{\mathrm{T}}, \\
\left|\mathbf{h}^{(k}\right| & =\left[\left|h_{1,1}^{(k}\right| \cdots\left|h_{1, N}^{(k}\right| \cdots\left|h_{M, 1}^{(k}\right| \cdots\left|h_{M, N}^{(k}\right|\right]^{\mathrm{T}} .
\end{aligned}
$$

Upon defining $\mathbf{h}=\left[\mathbf{h}_{1}^{\mathrm{T}} \mathbf{h}_{2}^{\mathrm{T}} \cdots \mathbf{h}_{K}^{\mathrm{T}}\right]^{\mathrm{T}}$ and the location vector 206 of the $K$ targets as $\boldsymbol{l}_{x, y}=\left[x_{1} y_{1} \cdots x_{K} y_{K}\right]^{\mathrm{T}}$, all the system's 207 parameters can be stacked into a single real-valued vector

$$
\mathbf{u}=\left[\boldsymbol{l}_{x, y}^{\mathrm{T}} \mathbf{h}^{\mathrm{T}}\right]^{\mathrm{T}} .
$$

Since the received signal (5) is also a function of the time delays 209 $\tau_{m, n}^{(k}$, we also define the following system parameter vector

$$
\boldsymbol{\psi}=\left[\tau_{1,1}^{(1} \cdots \tau_{1, N}^{(1} \cdots \tau_{M, N}^{(K} \mathbf{h}^{\mathrm{T}}\right]^{\mathrm{T}} .
$$

There exists a clear one-to-one relationship between $\mathbf{u}$ and $\boldsymbol{\psi} . \quad 211$

Let $f(\mathbf{r} \mid \mathbf{u})$ be the conditional probability density function 212 (PDF) of the observation vector $\mathbf{r}=\left[r_{1}(t), r_{2}(t), \cdots, r_{N}(t)\right] 213$ conditioned on $\mathbf{u}$. Similarly, we have the conditional PDF of $\mathbf{r} 214$ conditioned on $\boldsymbol{\psi}$. Then the unbiased estimate $\widehat{\mathbf{u}}$ of $\mathbf{u}$ satisfies 215 the following inequality [20]

$$
\mathrm{E}\left\{(\widehat{\mathbf{u}}-\mathbf{u})(\widehat{\mathbf{u}}-\mathbf{u})^{\mathrm{T}}\right\} \geq \mathbf{J}^{-1}(\mathbf{u}),
$$

where the Fisher information matrix (FIM) $\mathbf{J}(\mathbf{u})$ is defined by

$$
\mathbf{J}(\mathbf{u})=\mathrm{E}\left\{\frac{\partial}{\partial \mathbf{u}} \log f(\mathbf{r} \mid \mathbf{u})\left(\frac{\partial}{\partial \mathbf{u}} \log f(\mathbf{r} \mid \mathbf{u})\right)^{\mathrm{T}}\right\} .
$$

Similarly, we have the FIM of $\boldsymbol{\psi}$, denoted by $\mathbf{J}(\boldsymbol{\psi})$. The FIM 218 $\mathbf{J}(\mathbf{u})$ can be derived from $\mathbf{J}(\boldsymbol{\psi})$ according to

$$
\mathbf{J}(\mathbf{u})=\left[\begin{array}{cc}
\mathbf{D} & \mathbf{0} \\
\mathbf{0} & \mathbf{I}_{2 K M N}
\end{array}\right] \mathbf{J}(\boldsymbol{\psi})\left[\begin{array}{cc}
\mathbf{D} & \mathbf{0} \\
\mathbf{0} & \mathbf{I}_{2 K M N}
\end{array}\right]^{\mathrm{T}},
$$

where the $(2 K \times K M N)$-element block diagonal matrix $\mathbf{D} 220$ takes the following form

$$
\mathbf{D}=\operatorname{diag}\left(\mathbf{D}^{(1}, \mathbf{D}^{(2}, \cdots, \mathbf{D}^{(K}\right),
$$

with the $(2 \times M N)$-element sub-matrix $\mathbf{D}^{(k}$ given by

$$
\begin{aligned}
& \mathbf{D}^{(k}= {\left[\begin{array}{lll}
\frac{\partial \tau_{1,1}^{(k}}{\partial x_{k}} & \cdots & \frac{\partial \tau_{M, N}^{(k}}{\partial x_{k}} \\
\frac{\partial \tau_{1,1}^{(k}}{\partial y_{k}} & \cdots & \frac{\partial \tau_{M, N}^{(k}}{\partial y_{k}}
\end{array}\right] } \\
&=-\frac{1}{c}\left[\begin{array}{lll}
\cos \left(\theta_{1}^{(k)}\right)+\cos \left(\varphi_{1}^{(k)}\right) & \cdots & \cos \left(\theta_{M}^{(k)}\right)+\cos \left(\varphi_{N}^{(k)}\right) \\
\sin \left(\theta_{1}^{(k)}\right)+\sin \left(\varphi_{1}^{(k)}\right) & \cdots & \sin \left(\theta_{M}^{(k)}\right)+\sin \left(\varphi_{N}^{(k)}\right)
\end{array}\right] .
\end{aligned}
$$

The matrix $\mathbf{C}_{x, y}$ associated with the CRLB for the unbiased 223 estimator of $\boldsymbol{l}_{x, y}$ is the $(2 K \times 2 K)$-element upper left block 224 sub-matrix of $\mathbf{J}^{-1}(\mathbf{u})$, which can be derived as [11], [21] 225

$$
\mathbf{C}_{x, y}=\left[\mathbf{J}^{-1}(\mathbf{u})\right]_{[1: 2 K ; 1: 2 K]}=\left(\mathbf{D P} \boldsymbol{\Psi} \mathbf{D}^{\mathrm{T}}\right)^{-1},
$$


where $\mathbf{P}=\mathbf{I}_{K} \otimes \operatorname{diag}(\mathbf{p}) \otimes \mathbf{I}_{N}, \quad$ and $\quad \mathbf{\Psi}=\operatorname{diag}\left(\boldsymbol{\Psi}^{(1}, \cdots\right.$, $\left.\Psi^{(K)}\right)$ is the $(K M N \times K M N)$-element block diagonal matrix with the $k$ th sub-matrix defined as

$$
\boldsymbol{\Psi}^{(k}=8 \pi^{2}\left(\operatorname{diag}\left(\beta_{1}^{2}, \cdots, \beta_{M}^{2}\right) \otimes \mathbf{I}_{N}\right) \operatorname{diag}\left(\left|\mathbf{h}^{(k}\right|^{2}\right) .
$$

Let us denote the variances of the estimates of $x_{k}$ and $y_{k}$ by $\sigma_{x_{k}}^{2}$ and $\sigma_{y_{k}}^{2}$, respectively. Then we have

$$
\sum_{k=1}^{K}\left(\sigma_{x_{k}}^{2}+\sigma_{y_{k}}^{2}\right) \geq \operatorname{Tr}\left(\mathbf{C}_{x, y}\right),
$$

where $\operatorname{Tr}\left(\mathbf{C}_{x, y}\right)$ is a lower bound on the sum of the MSEs of the localization estimator $\widehat{\boldsymbol{l}}_{x, y}$. By defining $\mathbf{X}=\operatorname{diag}(\mathbf{p}) \otimes \mathbf{I}_{N}$ and noting $\mathbf{D}$ of (16), we obtain the expression of the lower bound for the $k$ th target location estimate as [12], [22]

$$
\begin{aligned}
\sum_{i=1}^{2} & {\left[\mathbf{C}_{x, y}\right]_{i+2(k-1), i+2(k-1)} } \\
& =\sum_{i=1}^{2}\left[\left(\mathbf{D P} \mathbf{\Psi} \mathbf{D}^{\mathrm{T}}\right)^{-1}\right]_{i+2(k-1), i+2(k-1)} \\
& =\operatorname{Tr}\left(\left[\begin{array}{ll}
\left(\mathbf{a}_{1,1}^{(k}\right)^{\mathrm{T}} \mathbf{p} & \left(\mathbf{a}_{1,2}^{(k}\right)^{\mathrm{T}} \mathbf{p} \\
\left(\mathbf{a}_{2,1}^{(k}\right)^{\mathrm{T}} \mathbf{p} & \left(\mathbf{a}_{2,2}^{(k}\right)^{\mathrm{T}} \mathbf{p}
\end{array}\right]^{-1}\right)=\frac{\mathbf{b}_{k}^{\mathrm{T}} \mathbf{p}}{\mathbf{p}^{\mathrm{T}} \mathbf{A}_{k} \mathbf{p}},
\end{aligned}
$$

where the second equation is obtained by first dividing the $(M N \times 2)$ matrix $\left(\mathbf{D}^{(k}\right)^{\mathrm{T}}$ into the two column vectors, $\left(\mathbf{D}^{(k}\right)^{\mathrm{T}}$ $=\left[\mathbf{d}_{1}^{(k} \mathbf{d}_{2}^{(k}\right]$, and generating the $(N \times 1)$ vectors

$$
\mathbf{d}_{i, m}^{(k}=\left[\mathbf{d}_{i}^{(k}\right]_{[(m-1) N+1: m N]}, i=1,2,1 \leq m \leq M .
$$

Then $\mathbf{a}_{i, j}^{(k}$ for $1 \leq i, j \leq 2$ are given by

$$
\begin{aligned}
\mathbf{a}_{i, j}^{(k}= & \mathbf{v}_{\operatorname{diag}}\left(\operatorname{diag}\left(\left(\mathbf{d}_{i, 1}^{(k}\right)^{\mathrm{T}}, \cdots,\left(\mathbf{d}_{i, M}^{(k)}\right)^{\mathrm{T}}\right) \mathbf{\Psi}^{(k}\right. \\
& \left.\times \operatorname{diag}\left(\mathbf{d}_{j, 1}^{(k}, \cdots, \mathbf{d}_{j, M}^{(k}\right)\right)
\end{aligned}
$$

while $\mathbf{b}_{k}=\mathbf{a}_{1,1}^{(k}+\mathbf{a}_{2,2}^{(k)}$ and $\mathbf{A}_{k}=\mathbf{a}_{1,1}^{(k}\left(\mathbf{a}_{2,2}^{(k)}\right)^{\mathrm{T}}-\mathbf{a}_{1,2}^{(k}\left(\mathbf{a}_{2,1}^{(k)}\right)^{\mathrm{T}}$.

Our task is to design a beneficial power allocation strategy capable of achieving a localization accuracy threshold $\eta$. We can use the weighting $v_{k}$ to indicate the localization accuracy requirement for the $k$ th target. The larger $v_{k}$ is, the higher accuracy is required for the $k$ th target. For a predetermined lower bound of total MSE of all the targets, the transmit power of the different transmit radars can then be appropriately allocated for minimizing the total transmit power. This can be formulated as the following optimization problem $\mathbb{P} 1$

$$
\begin{aligned}
& \min _{\mathbf{p}} \mathbf{1}^{\mathrm{T}} \mathbf{p} \\
& \mathbb{P} 1 \text { : } \quad \text { s.t. } \sum_{k=1}^{K} v_{k} \frac{\mathbf{b}_{k}^{\mathrm{T}} \mathbf{p}}{\mathbf{p}^{\mathrm{T}} \mathbf{A}_{k} \mathbf{p}} \leq \eta, \\
& p_{m_{\text {min }}} \leq p_{m} \leq p_{m_{\text {max }}}, 1 \leq m \leq M \text {. }
\end{aligned}
$$

Because generally speaking $\mathbf{A}_{k}$ is not a positive definite matrix, the optimization $\mathbb{P} 1$ is a nonconvex problem.
In [13], a similar optimization problem is formulated as

$$
\begin{array}{ll}
\min _{\mathbf{p}} & \mathbf{1}^{\mathrm{T}} \mathbf{p}, \\
\text { s.t. } & \frac{\mathbf{b}_{k}^{\mathrm{T}} \mathbf{p}}{\mathbf{p}^{\mathrm{T}} \mathbf{A}_{k} \mathbf{p}} \leq \bar{\eta}, 1 \leq k \leq K, \\
& p_{m_{\min }} \leq p_{m} \leq p_{m_{\max }}, 1 \leq m \leq M,
\end{array}
$$

given an equivalent localization accuracy threshold $\bar{\eta}$. In [13], 252 a Taylor series based technique is applied to approximate the 253 inequality constraints in (25) in order to relax the nonconvex 254 optimization problem for the sake of obtaining a solution. Intu- 255 itively, the cost function associated with an optimal solution of 256 our problem $\mathbb{P} 1$ of (24) is generally smaller than that associated 257 with an optimal solution of (25), i.e., we can achieve a lower 258 power consumption. This is achieved at the potential cost of a 259 slightly reduced localization accuracy.

\section{POWER RESOURCE AlLOCATION}

\section{A. Multi-Target Case}

In order to solve the nonconvex problem $\mathbb{P} 1$ of (24), we have 263 to change it into a simpler form. Specifically, we have to change the inequality constraint into an equality one, i.e.,

$$
\sum_{k=1}^{K} v_{k} \frac{\mathbf{b}_{k}^{\mathrm{T}} \mathbf{p}}{\mathbf{p}^{\mathrm{T}} \mathbf{A}_{k} \mathbf{p}} \leq \eta \Rightarrow \sum_{k=1}^{K} v_{k} \frac{\mathbf{b}_{k}^{\mathrm{T}} \mathbf{p}}{\mathbf{p}^{\mathrm{T}} \mathbf{A}_{k} \mathbf{p}}=\eta .
$$

Lemma 1: An increase of the transmit power $\mathbf{p}$ results in a 266 reduction of the MSE, namely,

$$
\sum_{k=1}^{K} v_{k} \frac{\mathbf{b}_{k}^{\mathrm{T}}(\mathbf{p}+\Delta \mathbf{p})}{(\mathbf{p}+\Delta \mathbf{p})^{\mathrm{T}} \mathbf{A}_{k}(\mathbf{p}+\Delta \mathbf{p})} \leq \sum_{k=1}^{K} v_{k} \frac{\mathbf{b}_{k}^{\mathrm{T}} \mathbf{p}}{\mathbf{p}^{\mathrm{T}} \mathbf{A}_{k} \mathbf{p}} .
$$

The proof of Lemma 1 is similar to that of single-target case 268 given in [12]. Thus, to achieve a reduced power consumption, 269 we can always set the MSE to its maximum tolerance. The 270 change of constraint as given in (26) leads to the problem $\mathbb{P} 2$,

$$
\begin{aligned}
& \min _{\mathbf{p}} \mathbf{1}^{\mathrm{T}} \mathbf{p}, \\
& \mathbb{P} 2 \text { : } \quad \text { s.t. } \sum_{k=1}^{K} v_{k} \frac{\mathbf{b}_{k}^{\mathrm{T}} \mathbf{p}}{\mathbf{p}^{\mathrm{T}} \mathbf{A}_{k} \mathbf{p}}=\eta \text {, } \\
& p_{m_{\text {min }}} \leq p_{m} \leq p_{m_{\text {max }}}, 1 \leq m \leq M \text {. }
\end{aligned}
$$

Theorem 1: The solutions of $\mathbb{P} 1$ and $\mathbb{P} 2$ are identical.

The proof of Theorem 1 is straightforward. By introducing the auxiliary variables

$$
w_{k}=\frac{1}{\eta \mathbf{p}^{\mathrm{T}} \mathbf{A}_{k} \mathbf{p}}, 1 \leq k \leq K,
$$

and their corresponding lower and upper bounds

$$
w_{k_{\min }}=\frac{1}{\eta \mathbf{p}_{\max }^{\mathrm{T}} \mathbf{A}_{k} \mathbf{p}_{\max }}, w_{k_{\max }}=\frac{1}{\eta \mathbf{p}_{\min }^{\mathrm{T}} \mathbf{A}_{k} \mathbf{p}_{\min }}, 1 \leq k \leq K,
$$


$\mathbb{P} 2$ is reformulated as the following optimization problem $\mathbb{P} 3$ :

$$
\begin{aligned}
& \min _{\mathbf{p}, \mathbf{w}} \mathbf{1}^{\mathrm{T}} \mathbf{p}, \\
& \text { s.t. } \sum_{k=1}^{K} v_{k} w_{k} \mathbf{b}_{k}^{\mathrm{T}} \mathbf{p}=1 \text {, } \\
& w_{k} \eta \mathbf{p}^{\mathrm{T}} \mathbf{A}_{k} \mathbf{p}=1,1 \leq k \leq K, \\
& p_{m_{\text {min }}} \leq p_{m} \leq p_{m_{\text {max }}}, 1 \leq m \leq M, \\
& w_{k_{\text {min }}} \leq w_{k} \leq w_{k_{\text {max }}}, 1 \leq k \leq K .
\end{aligned}
$$

The following corollary is obvious.

Corollary 1: If $\mathbf{p}^{\star}$ associated with $w_{k}^{\star}=\frac{1}{\eta\left(\mathbf{p}^{\star}\right)^{\mathrm{T}} \mathbf{A}_{k} \mathbf{p}^{\star}}$ for $1 \leq k \leq K$ is an optimal solution of the problem $\mathbb{P} 3(31)$, $\mathbf{p}^{\star}$ is an optimal solution for the problem $\mathbb{P} 1$ of (24). Conversely, if $\mathbf{p}^{\star}$ is an optimal solution of the problem $\mathbb{P} 1$, together with $w_{k}^{\star}=\frac{1}{\eta\left(\mathbf{p}^{\star}\right)^{\mathrm{T}} \mathbf{A}_{k} \mathbf{p}^{\star}}$ for $1 \leq k \leq K$ it is an optimal solution of the problem $\mathbb{P} 3$.

1) OCD-based method: The Lagrangian associated with the optimization problem $\mathbb{P} 3$ is

$$
\begin{aligned}
L(\mathbf{p}, \mathbf{w}, \lambda, \boldsymbol{\mu})= & \mathbf{1}^{\mathrm{T}} \mathbf{p}+\lambda\left(\sum_{k=1}^{K} v_{k} w_{k} \mathbf{b}_{k}^{\mathrm{T}} \mathbf{p}-1\right) \\
& +\sum_{k=1}^{K} \mu_{k}\left(w_{k} \eta \mathbf{p}^{\mathrm{T}} \mathbf{A}_{k} \mathbf{p}-1\right),
\end{aligned}
$$

with $\mathbf{w}=\left[w_{1} w_{2} \cdots w_{K}\right]^{\mathrm{T}}$ and $\boldsymbol{\mu}=\left[\mu_{1} \mu_{2} \cdots \mu_{K}\right]^{\mathrm{T}}$, where $\lambda$ and $\mu_{k}$ for $1 \leq k \leq K$ are Lagrangian multipliers. We optimize the Lagrangian (32) with respect to $\mathbf{p}, \lambda, w_{k}$ and $\mu_{k}$. Using the steepest descent method, the search directions are related to the Karush-Kuhn-Tucker (KKT) conditions by

$$
\begin{aligned}
\Delta \mathbf{p}= & \nabla_{\mathbf{p}} L(\mathbf{p}, \mathbf{w}, \lambda, \boldsymbol{\mu})=\mathbf{1}+\lambda\left(\sum_{k=1}^{K} w_{k} v_{k} \mathbf{b}_{k}\right) \\
& +\sum_{k=1}^{K} \mu_{k} w_{k} \eta\left(\mathbf{A}_{k}+\mathbf{A}_{k}^{\mathrm{T}}\right) \mathbf{p}, \\
\Delta \lambda= & \nabla_{-\lambda} L(\mathbf{p}, \mathbf{w}, \lambda, \boldsymbol{\mu})=-\sum_{k=1}^{K} w_{k} v_{k} \mathbf{b}_{k}^{\mathrm{T}} \mathbf{p}+1, \\
\Delta w_{k}= & \nabla_{w_{k}} L(\mathbf{p}, \mathbf{w}, \lambda, \boldsymbol{\mu}) \\
= & \lambda v_{k} \mathbf{b}_{k}^{\mathrm{T}} \mathbf{p}+\mu_{k} \eta \mathbf{p}^{\mathrm{T}} \mathbf{A}_{k} \mathbf{p}, 1 \leq k \leq K, \\
\Delta \mu_{k}= & \nabla_{-\mu_{k}} L(\mathbf{p}, \mathbf{w}, \lambda, \boldsymbol{\mu}) \\
= & -\left(\eta w_{k} \mathbf{p}^{\mathrm{T}} \mathbf{A}_{k} \mathbf{p}+1\right), 1 \leq k \leq K,
\end{aligned}
$$

291 where we have $\Delta \mathbf{p}=\left[\Delta p_{1} \Delta p_{2} \cdots \Delta p_{M}\right]^{\mathrm{T}}$. The primal and

$$
\begin{aligned}
& p_{m}^{(n+1)}=\left[p_{m}^{(n)}-\kappa_{1} \Delta p_{m}^{(n)}\right]_{p_{m_{\min }}}^{p_{m_{\max }}}, 1 \leq m \leq M, \\
& \lambda^{(n+1)}=\lambda^{(n)}-\kappa_{2} \Delta \lambda^{(n)}, \\
& w_{k}^{(n+1)}=w_{k}^{(n)}-\kappa_{3} \Delta w_{k}^{(n)}, 1 \leq k \leq K, \\
& \mu_{k}^{(n+1)}=\mu_{k}^{(n)}-\kappa_{4} \Delta \mu_{k}^{(n)}, 1 \leq k \leq K,
\end{aligned}
$$

where the superscript ${ }^{(n)}$ denotes the iteration index and

$$
[a]_{b}^{c}=\min \{\max \{a, b\}, c\},
$$

while $\kappa_{i}$ for $1 \leq i \leq 4$ represent the step sizes for the primal 294 variables $\mathbf{p}$, the dual variable $\lambda$, the primal variables $\mathbf{w}$ and the 295 dual variables $\boldsymbol{\mu}$, respectively. According to [23], an exponen- 296 tially decreasing step size is highly desired. Furthermore, since 297 $\mathrm{p}, \lambda, \mathbf{w}$ and $\boldsymbol{\mu}$ have very different properties and their impacts 298 on the Lagrangian are 'unequal', using different step sizes for 299 them makes sense. By combining these two considerations, we 300 set the four step sizes for $\mathbf{p}, \lambda$, w and $\boldsymbol{\mu}$ according to

$$
\kappa_{i}=c_{i} e^{-\alpha n} \text { with } 0 \leq \alpha \ll 1 \text {, for } 1 \leq i \leq 4,
$$

where $c_{i}>0$ for $1 \leq i \leq 4$ are different constants.

The choice of the initial values for the primal variables $p_{m}, \quad 303$ $1 \leq m \leq M$, influences the convergence performance. Ideally, 304 they should be chosen to be close to their own specific optimal 305 values so as to enhance the convergence speed. For practical 306 reason, the initialization should be easy and simple to realize 307 too. Hence we opt for the initial powers of

$$
\mathbf{p}^{(0)}=\mathbf{p}_{\text {equ }}=\frac{1}{\eta} \sum_{k=1}^{K} v_{k} \frac{\mathbf{b}_{k}^{\mathrm{T}} \mathbf{1}}{\mathbf{1}^{\mathrm{T}} \mathbf{A}_{k} \mathbf{1}} \mathbf{1},
$$

which is obtained by setting all the elements of $\mathbf{p}$ to be equal. 309 Then, $w_{k}$ is initialized according to

$$
w_{k}^{(0)}=\frac{1}{\eta \mathbf{p}_{\text {equ }}^{\mathrm{T}} \mathbf{A}_{k} \mathbf{p}_{\text {equ }}}, 1 \leq k \leq K .
$$

The iterative procedure of (37) to (40) is repeated until 311 $\left\|\mathbf{p}^{(n+1)}-\mathbf{p}^{(n)}\right\|$ becomes smaller than a preset small positive number or the maximum number of iterations is reached.

Remark 1: It is difficult to find a closed-form solution from the set of KKT conditions, because $\mathbf{A}_{k}$ for $1 \leq k \leq K$ are generally non-invertible. Hence our algorithm finds a locally optimal point in an iterative manner. It is also worth noting that the standard OCD [17] is typically based on a Newtontype algorithm, but our proposed OCD method is a steepest descent algorithm. The reason is that the Hessian matrix for the Lagrangian $L(\mathbf{p}, \mathbf{w}, \lambda, \boldsymbol{\mu})$ of (32) is not invertible.

2) ADMM-based method: ADMM was originally proposed for solving convex problems in a parallel manner [18]. Let us now discuss how to apply the ADMM method for solving the nonconvex problem $\mathbb{P} 3$. By introducing an auxiliary vector $\mathbf{z}=$ p, (29) can be rewritten as

$$
\mathbf{p}=\mathbf{z} \text { and } \eta w_{k} \mathbf{z}^{\mathrm{T}} \mathbf{A}_{k} \mathbf{p}=1,1 \leq k \leq K .
$$

Therefore, $\mathbb{P} 3$ can be reformulated into the problem $\mathbb{P} 4$ :

$\mathbb{P} 4$ :

$$
\begin{aligned}
\min _{\mathbf{p}, \mathbf{w}, \mathbf{z}} & \mathbf{1}^{\mathrm{T}} \mathbf{p}, \\
\text { s.t. } & \sum_{k=1}^{K} v_{k} w_{k} \mathbf{b}_{k}^{\mathrm{T}} \mathbf{p}=1, \\
& \mathbf{p}=\mathbf{z}, \\
& w_{k} \eta \mathbf{z}^{\mathrm{T}} \mathbf{A}_{k} \mathbf{p}=1,1 \leq k \leq K, \\
& p_{m_{\min }} \leq p_{m} \leq p_{m_{\max }}, 1 \leq m \leq M, \\
& w_{k_{\min }} \leq w_{k} \leq w_{k_{\max }}, 1 \leq k \leq K .
\end{aligned}
$$


This problem is convex with respect to $\mathbf{p}, \mathbf{z}$ and $w_{k}$, respectively. An augmented Lagrangian is constructed as follows

$$
\begin{aligned}
& L\left(\mathbf{p}, \mathbf{w}, \mathbf{z}, \mathbf{d}_{0}, d_{1}, \mathbf{d}_{2}\right)=\mathbf{1}^{\mathrm{T}} \mathbf{p}+\frac{\rho_{0}}{2}\|\mathbf{p}-\mathbf{z}\|^{2}+\mathbf{d}_{0}^{\mathrm{T}}(\mathbf{p}-\mathbf{z}) \\
& +\sum_{k=1}^{K} \frac{\rho_{2, k}}{2}\left|w_{k} \mathbf{z}^{\mathrm{T}} \mathbf{A}_{k} \mathbf{p} \eta-1\right|^{2}+\sum_{k=1}^{K} d_{2, k}\left(w_{k} \mathbf{z}^{\mathrm{T}} \mathbf{A}_{k} \mathbf{p} \eta-1\right) \\
& +\frac{\rho_{1}}{2}\left|\sum_{k=1}^{K} w_{k} v_{k} \mathbf{b}_{k}^{\mathrm{T}} \mathbf{p}-1\right|^{2}+d_{1}\left(\sum_{k=1}^{K} w_{k} v_{k} \mathbf{b}_{k}^{\mathrm{T}} \mathbf{p}-1\right)
\end{aligned}
$$

where $\mathbf{d}_{0}=\left[d_{0,1} \cdots d_{0, M}\right]^{\mathrm{T}}, d_{1}$ and $\mathbf{d}_{2}=\left[d_{2,1} \cdots d_{2, K}\right]^{\mathrm{T}}$ are the dual variables corresponding to the constraints $\mathbf{p}=\mathbf{z}$, $\sum_{k=1}^{K} w_{k} v_{k} \mathbf{b}_{k}^{\mathrm{T}} \mathbf{p}=1$ and $w_{k} \mathbf{z}^{\mathrm{T}} \mathbf{A}_{k} \mathbf{p} \eta=1$ for $1 \leq k \leq K$, respectively, while $\rho_{0}, \rho_{1}$ and $\boldsymbol{\rho}_{2}=\left[\rho_{2,1} \cdots \rho_{2, K}\right]^{\mathrm{T}}$ are the penalty parameters. Note that the augmented Lagrangian (47) is quadratic. For convenience, we scale the dual variables as $\mathbf{e}=\frac{1}{\rho_{0}} \mathbf{d}_{0}, \mu=\frac{1}{\rho_{1}} d_{1}$ and $\gamma=\left[\gamma_{1} \cdots \gamma_{K}\right]^{\mathrm{T}}$ with $\gamma_{k}=\frac{1}{\rho_{2, k}} d_{2, k}$ for $1 \leq k \leq K$. Then, from (47) we obtain the following augmented Lagrangian

$$
\begin{gathered}
L(\mathbf{p}, \mathbf{w}, \mathbf{z}, \mathbf{e}, \mu, \gamma)=\mathbf{1}^{\mathrm{T}} \mathbf{p}+\frac{\rho_{0}}{2}\|\mathbf{p}-\mathbf{z}+\mathbf{e}\|^{2}-\frac{\rho_{0}}{2}\|\mathbf{e}\|^{2} \\
+\sum_{k=1}^{K} \frac{\rho_{2, k}}{2}\left|w_{k} \mathbf{z}^{\mathrm{T}} \mathbf{A}_{k} \mathbf{p} \eta-1+\gamma_{k}\right|^{2}-\sum_{k=1}^{K} \frac{\rho_{2, k}}{2}\left|\gamma_{k}\right|^{2} \\
+\frac{\rho_{1}}{2}\left|\sum_{k=1}^{K} w_{k} v_{k} \mathbf{b}_{k}^{\mathrm{T}} \mathbf{p}-1+\mu\right|^{2}-\frac{\rho_{1}}{2}|\mu|^{2}
\end{gathered}
$$

We can find the saddle point of the augmented Lagrangian (48) by minimizing the Lagrangian over the primal variables $\mathbf{p}, \mathbf{w}$ and $\mathbf{z}$, as well as maximizing it over the dual variables $\mathbf{e}, \mu$ and $\gamma$, in an alternative way. In particular, we update the primal variables $\mathbf{p}, \mathbf{w}$ and $\mathbf{z}$ separately one by one. Furthermore, after the update of the dual variables e, $\mu$ and $\gamma$, we adjust the penalty parameters $\rho_{0}, \rho_{1}$ and $\rho_{2}$. We now summarize our ADMMbased procedure.

Initialization: Let us also opt for the equal power initialization $\mathbf{p}^{(0)}=\mathbf{p}_{e q u}$ of (43). The other primal variables are initialized as $w_{k}^{(0)}=\frac{1}{\eta \mathbf{p}_{e q u}^{\mathrm{T}} \mathbf{A}_{k} \mathbf{p}_{e q u}}$ for $1 \leq k \leq K$ of (44), and

$$
\mathbf{z}^{(0)}=\mathbf{p}_{e q u} .
$$

The initial penalty parameters, $\rho_{0}^{(0)}, \rho_{1}^{(0)}$ and $\rho_{2, k}^{(0)}$ for $1 \leq k \leq$ $K$, are typically set to a large positive value, say, 500 . Next, the dual variables are initialized as follows. Choose $\mu^{(0)}=1$ and $\gamma_{k}^{(0)}=1$ for $1 \leq k \leq K$, while every element of $\mathbf{e}^{(0)}$ is set to 1 too. Then we set the iteration index $n=0$.

Iterative Procedure: At the $(n+1)$ th iteration, perform:
- Step 1: Update the primal variables p. Upon isolating all 356 the terms involving $\mathbf{p}$ in the Lagrangian (48), we have

$$
\begin{aligned}
\min _{\mathbf{p}} & \mathbf{1}^{\mathrm{T}} \mathbf{p}+\frac{\rho_{0}^{(n)}}{2}\left\|\mathbf{p}-\mathbf{z}^{(n)}+\mathbf{e}^{(n)}\right\|^{2} \\
& +\frac{\rho_{1}^{(n)}}{2}\left|\sum_{k=1}^{K} w_{k}^{(n)} v_{k} \mathbf{b}_{k}^{\mathrm{T}} \mathbf{p}-1+\mu^{(n)}\right|^{2} \\
& +\sum_{k=1}^{K} \frac{\rho_{2, k}^{(n)}}{2}\left|w_{k}^{(n)}\left(\mathbf{z}^{(n)}\right)^{\mathrm{T}} \mathbf{A}_{k} \mathbf{p} \eta-1+\gamma_{k}^{(n)}\right|^{2}, \\
\text { s.t. } & p_{m_{\min }} \leq p_{m} \leq p_{m_{\max }}, 1 \leq m \leq M,
\end{aligned}
$$

which is a constrained convex optimization. Setting the 358 derivative of the objective function to zero yields the $(n+359$ 1)th estimate of $\mathbf{p}$ as follows. First compute

$$
\begin{aligned}
\overline{\mathbf{p}}^{(n+1)=} & {\left[\bar{p}_{1}^{(n+1)} \cdots \bar{p}_{M}^{(n+1)}\right]^{\mathrm{T}}=\left(\mathbf{P}_{1}^{(n+1)}\right)^{-1} \mathbf{p}_{2}^{(n+1)}, } \\
\mathbf{P}_{1}^{(n+1)}= & \rho_{0}^{(n)} \mathbf{I}_{M}+\rho_{1}^{(n)}\left(\sum_{k=1}^{K} w_{k}^{(n)} v_{k} \mathbf{b}_{k}\right) \\
& \times\left(\sum_{k=1}^{K} w_{k}^{(n)} v_{k} \mathbf{b}_{k}^{\mathrm{T}}\right)+\sum_{k=1}^{K} \rho_{2, k}^{(n)} \\
& \times\left(w_{k}^{(n)}\left(\mathbf{A}_{k}\right)^{\mathrm{T}} \mathbf{z}^{(n)} \eta\right)\left(w_{k}^{(n)}\left(\mathbf{z}^{(n)}\right)^{\mathrm{T}} \mathbf{A}_{k} \eta\right)^{\mathrm{T}}, \\
\mathbf{p}_{2}^{(n+1)}= & -\mathbf{1}+\rho_{0}^{(n)}\left(\mathbf{z}^{(n)}+\mathbf{e}^{(n)}\right) \\
& +\rho_{1}^{(n)}\left(\sum_{k=1}^{K} w_{k}^{(n)} v_{k} \mathbf{b}_{k}\right)\left(1-\mu^{(n)}\right) \\
& +\rho_{2, k}^{(n)}\left(w_{k}^{(n)}\left(\mathbf{A}_{k}\right)^{\mathrm{T}} \mathbf{z}^{(n)} \eta\right)\left(1-\gamma_{k}^{(n)}\right) . \quad(53)
\end{aligned}
$$

The final estimate is then given by

$$
p_{m}^{(n+1)}=\left[\bar{p}_{m}^{(n+1)}\right]_{p_{m \text { min }}}^{p_{m \text { max }}}, 1 \leq m \leq M .
$$

- Step 2: Update the primal variables $\mathrm{w}$. The optimization involving $\mathbf{w}$ is also a constrained convex problem

$$
\begin{aligned}
\min _{\mathbf{w}} & \frac{\rho_{1}^{(n)}}{2}\left|\sum_{k=1}^{K} w_{k} v_{k} \mathbf{b}_{k}^{\mathrm{T}} \mathbf{p}^{(n+1)}-1+\mu^{(n)}\right|^{2} \\
& +\sum_{k=1}^{K} \frac{\rho_{2, k}^{(n)}}{2}\left|w_{k}\left(\mathbf{z}^{(n)}\right)^{\mathrm{T}} \mathbf{A}_{k} \mathbf{p}^{(n+1)} \eta-1+\gamma_{k}^{(n)}\right|^{2}, \\
\text { s.t. } & w_{k_{\mathrm{min}}} \leq w_{k} \leq w_{k_{\max }}, 1 \leq k \leq K .
\end{aligned}
$$

The solution is given by

$$
w_{k}^{(n+1)}=\left[\frac{w_{k, 1}^{(n+1)}}{w_{k, 2}^{(n+1)}}\right]_{w_{k_{\mathrm{m} \text { in }}}}^{w_{k_{\mathrm{max}}}}, 1 \leq k \leq K,
$$


where

$$
\begin{aligned}
w_{k, 1}^{(n+1)}= & \rho_{1}^{(n)} v_{k} \mathbf{b}_{k}^{\mathrm{T}} \mathbf{p}^{(n+1)}\left(1-\mu^{(n)}-\sum_{k^{\prime} \neq k} v_{k^{\prime}} \mathbf{b}_{k^{\prime}}^{\mathrm{T}} \mathbf{p}^{(n+1)}\right) \\
& +\rho_{2, k}^{(n)}\left(\left(\mathbf{z}^{(n)}\right)^{\mathrm{T}} \mathbf{A}_{k} \mathbf{p}^{(n+1)} \eta\right)\left(1-\gamma_{k}^{(n)}\right) \\
w_{k, 2}^{(n+1)}= & \rho_{1}^{(n)}\left(v_{k} \mathbf{b}_{k}^{\mathrm{T}} \mathbf{p}^{(n+1)}\right)^{2} \\
& +\rho_{2, k}^{(n)}\left(\left(\mathbf{z}^{(n)}\right)^{\mathrm{T}} \mathbf{A}_{k} \mathbf{p}^{(n+1)} \eta\right)^{2} .
\end{aligned}
$$

- Step 3: Update the primal variables z. Isolating all the terms involving $\mathbf{z}$, the optimization is an unconstrained convex problem

$$
\begin{aligned}
\min _{\mathbf{z}} & \frac{\rho_{0}^{(n)}}{2}\left\|\mathbf{p}^{(n+1)}-\mathbf{z}+\mathbf{e}^{(n)}\right\|^{2} \\
& +\sum_{k=1}^{K} \frac{\rho_{2, k}^{(n)}}{2}\left|w_{k}^{(n+1)} \mathbf{z}^{\mathrm{T}} \mathbf{A}_{k} \mathbf{p}^{(n+1)} \eta-1+\gamma_{k}^{(n)}\right|^{2} .
\end{aligned}
$$

Solving (59) yields the $(n+1)$ th estimate of $\mathbf{z}$ as

$$
\mathbf{z}^{(n+1)}=\left(\mathbf{Z}_{1}^{(n+1)}\right)^{-1} \mathbf{z}_{2}^{(n+1)}
$$

where

$$
\begin{aligned}
\mathbf{Z}_{1}^{(n+1)}= & \rho_{0}^{(n)} \mathbf{I}_{M}+\sum_{k=1}^{K} \rho_{2, k}^{(n)}\left(w_{k}^{(n+1)} \mathbf{A}_{k} \mathbf{p}^{(n+1)} \eta\right) \\
& \times\left(w_{k}^{(n+1)} \mathbf{A}_{k} \mathbf{p}^{(n+1)} \eta\right)^{\mathrm{T}}, \\
\mathbf{z}_{2}^{(n+1)}= & \rho_{0}^{(n)}\left(\mathbf{p}^{(n+1)}+\mathbf{e}^{(n)}\right) \\
& +\sum_{k=1}^{K} \rho_{2, k}^{(n)}\left(w_{k}^{(n+1)} \mathbf{A}_{k} \mathbf{p}^{(n+1)} \eta\right)\left(1-\gamma_{k}^{(n)}\right) .
\end{aligned}
$$

- Step 4: Update the dual variables e, $\mu$ and $\gamma$. Maximizing the Lagrangian (48) with respect to the dual variables yields

$$
\begin{aligned}
\mathbf{e}^{(n+1)}= & \mathbf{e}^{(n)}+\mathbf{p}^{(n+1)}-\mathbf{z}^{(n+1)} \\
\mu^{(n+1)}= & \mu^{(n)}+\sum_{k=1}^{K} w_{k}^{(n+1)} v_{k} \mathbf{b}_{k}^{\mathrm{T}} \mathbf{p}^{(n+1)}-1 \\
\gamma_{k}^{(n+1)}= & \gamma_{k}^{(n)}+w_{k}^{(n+1)}\left(\mathbf{z}^{(n+1)}\right)^{\mathrm{T}} \mathbf{A}_{k} \mathbf{p}^{(n+1)} \eta-1 \\
& 1 \leq k \leq K
\end{aligned}
$$

- Step 5: Update the penalty parameters $\rho_{0}, \rho_{1}$ and $\rho_{2}$. The penalty parameters are updated at the end of each iteration for the first a few iterations to speed up the convergence. At the $(n+1)$ th iteration, associated with the three penalty parameters of $\rho_{0}^{(n)}, \rho_{1}^{(n)}$ and $\boldsymbol{\rho}_{2}^{(n)}$, we have three primal residuals

$$
\begin{aligned}
r_{0}^{(n+1)}= & \left\|\mathbf{p}^{(n+1)}-\mathbf{z}^{(n+1)}\right\|, \\
r_{1}^{(n+1)}= & \left|\sum_{k=1}^{K} w_{k}^{(n+1)} v_{k} \mathbf{b}_{k}^{\mathrm{T}} \mathbf{p}^{(n+1)}-1\right|, \\
r_{2, k}^{(n+1)}= & \left|w_{k}\left(\mathbf{z}^{(n+1)}\right)^{\mathrm{T}} \mathbf{A}_{k} \mathbf{p}^{(n+1)} \eta-1\right|, \\
& 1 \leq k \leq K,
\end{aligned}
$$

as well as three respective dual residuals

$$
\begin{aligned}
& s_{0}^{(n+1)}=\left\|\rho_{0}^{(n)}\left(\mathbf{z}^{(n+1)}-\mathbf{z}^{(n)}\right)\right\|, \\
& s_{1}^{(n+1)}=\left\|\mathbf{s}_{1 a}^{(n+1)}\right\|, \\
& s_{2, k}^{(n+1)}=\sqrt{\left(s_{2 a, k}^{(n+1)}\right)^{2}+\left\|\mathbf{s}_{2 b, k}^{(n+1)}\right\|}, 1 \leq k \leq K,
\end{aligned}
$$

where

$$
\begin{aligned}
\mathbf{s}_{1 a}^{(n+1)}= & \mu^{(n+1)} \rho_{1}^{(n)}\left(\sum_{k=1}^{K}\left(w_{k}^{(n)}-w_{k}^{(n+1)}\right) v_{k} \mathbf{b}_{k}\right) \\
& +\rho_{1}^{(n)}\left(\sum_{k=1}^{K} w_{k}^{(n)} v_{k} \mathbf{b}_{k}\right) \\
& \times\left(\sum_{k=1}^{K}\left(w_{k}^{(n)}-w_{k}^{(n+1)}\right) v_{k} \mathbf{b}_{k}^{\mathrm{T}} \mathbf{p}^{(n+1)}\right),
\end{aligned}
$$

$$
\begin{aligned}
s_{2 a, k}^{(n+1)}= & \rho_{2, k}^{(n)}\left(\mathbf{z}^{(n)}\right)^{\mathrm{T}} \mathbf{A}_{k} \mathbf{p}^{(n+1)} \eta \\
& \times\left(w_{k}^{(n+1)}\left(\mathbf{z}^{(n)}-\mathbf{z}^{(n+1)}\right)^{\mathrm{T}} \mathbf{A}_{k} \mathbf{p}^{(n+1)} \eta-1\right) \\
& +\gamma_{k}^{(n+1)} \rho_{2, k}^{(n)}\left(\left(\mathbf{z}^{(n)}-\mathbf{z}^{(n+1)}\right)^{\mathrm{T}} \mathbf{A}_{k} \mathbf{p}^{(n+1)} \eta\right), \\
\mathbf{s}_{2 b, k}^{(n+1)}= & \rho_{2, k}^{(n)} w_{k}^{(n)} \eta \mathbf{A}_{k}^{\mathrm{T}} \mathbf{z}^{(n)} \\
& \times\left(\left(w_{k}^{(n)}\left(\mathbf{z}^{(n)}\right)^{\mathrm{T}}-w_{k}^{(n+1)}\left(\mathbf{z}^{(n+1)}\right)^{\mathrm{T}}\right)\right. \\
& \left.\times \mathbf{A}_{k} \mathbf{p}^{(n+1)} \eta\right)+\gamma_{k}^{(n+1)} \rho_{2, k}^{(n)} \eta \mathbf{A}_{k}^{\mathrm{T}} \\
& \times\left(w_{k}^{(n)} \mathbf{z}^{(n)}-w_{k}^{(n+1)} \mathbf{z}^{(n+1)}\right) .
\end{aligned}
$$

The exact definitions of the dual residuals can be found in 381 Appendix A.

The penalty parameter $\rho_{0}$ is updated as follows

$$
\rho_{0}^{(n+1)}= \begin{cases}\tau \rho_{0}^{(n)}, & \text { if } r_{0}^{(n+1)} \geq \varepsilon s_{0}^{(n+1)}, \\ \frac{1}{\tau} \rho_{0}^{(n)}, & \text { if } s_{0}^{(n+1)} \geq \varepsilon r_{0}^{(n+1)}, \\ \rho_{0}^{(n)}, & \text { otherwise, }\end{cases}
$$

where the scalars $\tau>1$ and $\varepsilon>1$ with typical values of 384 $\tau=2$ and $\varepsilon=10$. The idea behind this penalty parameter 385 update is to balance the primal and dual residual norms 386 $r_{0}^{(n+1)}$ and $s_{0}^{(n+1)}$, i.e., to keep $\frac{r_{0}^{(n+1)}}{s_{0}^{(n+1)}} \leq \varepsilon$ and $\frac{s_{0}^{(n+1)}}{r_{0}^{(n+1)}} \leq \varepsilon, \quad 387$ 
as they both converge to zero [18], [25]. The related dual variables are rescaled to remove the impact of changing $\rho_{0}$ according to

$$
\mathbf{e}^{(n+1)}=\frac{\rho_{0}^{(n)}}{\rho_{0}^{(n+1)}} \mathbf{e}^{(n)} .
$$

Similarly, $\rho_{1}$ is updated according to

$$
\rho_{1}^{(n+1)}=\left\{\begin{array}{cl}
\tau \rho_{1}^{(n)}, & \text { if } r_{1}^{(n+1)} \geq \varepsilon s_{1}^{(n+1)}, \\
\frac{1}{\tau} \rho_{1}^{(n)}, & \text { if } s_{1}^{(n+1)} \geq \varepsilon r_{1}^{(n+1)}, \\
\rho_{1}^{(n)}, & \text { otherwise. }
\end{array}\right.
$$

The related dual variable is then scaled according to

$$
\mu^{(n+1)}=\frac{\rho_{1}^{(n)}}{\rho_{1}^{(n+1)}} \mu^{(n)} .
$$

Likewise, $\rho_{2, k}$ for $1 \leq k \leq K$ are updated according to

$$
\rho_{2, k}^{(n+1)}=\left\{\begin{array}{cl}
\tau \rho_{2, k}^{(n)}, & \text { if } r_{2, k}^{(n+1)} \geq \varepsilon s_{2, k}^{(n+1)}, \\
\frac{1}{\tau} \rho_{2, k}^{(n)}, & \text { if } s_{2, k}^{(n+1)} \geq \varepsilon r_{2, k}^{(n+1)}, \\
\rho_{2, k}^{(n)}, & \text { otherwise, }
\end{array},\right.
$$

and the corresponding dual variables are rescaled as

$$
\gamma_{k}^{(n+1)}=\frac{\rho_{2, k}^{(n)}}{\rho_{2, k}^{(n+1)}} \gamma_{k}^{(n)}, 1 \leq k \leq K .
$$

- Termination of the iterative procedure. The iterative procedure is terminated when $\left\|\mathbf{p}^{(n+1)}-\mathbf{p}^{(n)}\right\|$ becomes smaller than a predefined small positive value or the preset maximum number of iterations is reached. Otherwise, set $n=n+1$ and go to Step 1 .

Remark 2: The ADMM combines the advantages of the dual ascent and the augmented Lagrangian method. The dual ascent approach deals with the complicated constraints, while the augmented Lagrangian method is capable of enhancing the convergence rate and the robustness of the algorithm.

Remark 3: We deal with the optimization problem (24), and in every iteration of our OCD and ADMM methods, we have a closed-form update value. By contrast, Garcia et al. [13] deal with the optimization problem (25), and in every iteration, an inner iterative loop is required for computing an updated value by the algorithm of [13].

\section{B. Single-Target Case}

The target index $k$ can be dropped and then the optimization is simplified to the problem $\mathbb{P} 5$

$$
\begin{aligned}
\min _{\mathbf{p}} & \mathbf{1}^{\mathrm{T}} \mathbf{p}, \\
\mathbb{P} 5: \quad \text { s.t. } & \frac{\mathbf{b}^{\mathrm{T}} \mathbf{p}}{\mathbf{p}^{\mathrm{T}} \mathbf{A} \mathbf{p}} \leq \eta, \\
& p_{m_{\min }} \leq p_{m} \leq p_{m_{\max }}, 1 \leq m \leq M .
\end{aligned}
$$

equivalent to the optimization problem $\mathbb{P} 6$ :

$$
\begin{aligned}
& \min _{\mathbf{p}, w} \mathbf{1}^{\mathrm{T}} \mathbf{p},
\end{aligned}
$$

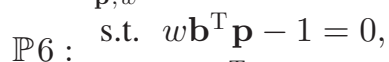

$$
\begin{aligned}
& w \eta \mathbf{p}^{\mathrm{T}} \mathbf{A} \mathbf{p}-1=0, \\
& p_{m_{\text {min }}} \leq p_{m} \leq p_{m_{\text {max }}}, 1 \leq m \leq M \text {. }
\end{aligned}
$$

This problem is nonconvex due to its equality constraint.

1) OCD-based method: The Lagrangian of (82) is

$L(\mathbf{p}, w, \lambda, \mu)=\mathbf{1}^{\mathrm{T}} \mathbf{p}+\lambda\left(w \mathbf{b}^{\mathrm{T}} \mathbf{p}-1\right)+\mu\left(w \eta \mathbf{p}^{\mathrm{T}} \mathbf{A} \mathbf{p}-1\right)$,

where $\lambda$ and $\mu$ are the dual variables. The gradients of this 419 Lagrangian are given by

$$
\begin{aligned}
& \Delta \mathbf{p}=\nabla_{\mathbf{p}} L(\mathbf{p}, w, \lambda, \mu)=\mathbf{1}+\lambda(w \mathbf{b})+\mu w \eta\left(\mathbf{A}+\mathbf{A}^{\mathrm{T}}\right) \mathbf{p} \\
& \Delta \lambda=\nabla_{-\lambda} L(\mathbf{p}, w, \lambda, \mu)=-w \mathbf{b}^{\mathrm{T}} \mathbf{p}+1 \\
& \Delta w=\nabla_{w} L(\mathbf{p}, w, \lambda, \mu)=\lambda \mathbf{b}^{\mathrm{T}} \mathbf{p}+\mu \eta \mathbf{p}^{\mathrm{T}} \mathbf{A} \mathbf{p} \\
& \Delta \mu=\nabla_{-\mu} L(\mathbf{p}, w, \lambda, \mu)=-\eta w \mathbf{p}^{\mathrm{T}} \mathbf{A} \mathbf{p}-1
\end{aligned}
$$

Given $\lambda^{(0)}, \mu^{(0)}$ and

$$
\mathbf{p}^{(0)}=\mathbf{p}_{e q u}=\frac{1}{\eta} \frac{\mathbf{b}^{\mathrm{T}} \mathbf{1}}{\mathbf{1}^{\mathrm{T}} \mathbf{A} \mathbf{1}} \mathbf{1},
$$

$\mathbf{p}, \lambda, w, \mu$ are updated in the following iterative procedure

$$
\begin{aligned}
p_{m}^{(n+1)} & =\left[p_{m}^{(n)}-\kappa_{1} \Delta p_{m}^{(n)}\right]_{p_{m_{\min }}}^{p_{m_{\max }}}, 1 \leq m \leq M, \\
\lambda^{(n+1)} & =\lambda^{(n)}-\kappa_{2} \Delta \lambda^{(n)}, \\
w^{(n+1)} & =w^{(n)}-\kappa_{3} \Delta w^{(n)}, \\
\mu^{(n+1)} & =\mu^{(n)}-\kappa_{4} \Delta \mu^{(n)},
\end{aligned}
$$

where again the step sizes are chosen according to (42). The 423 iterative procedure is repeated until $\left\|\mathbf{p}^{(n+1)}-\mathbf{p}^{(n)}\right\|$ becomes 424 smaller than a preset threshold.

2) ADMM-based method: Similar to the multi-target case, we reformulate the problem $\mathbb{P} 6$ as

$$
\begin{aligned}
\min _{\mathbf{p}, \mathbf{z}} & \mathbf{1}^{\mathrm{T}} \mathbf{p}, \\
\text { s.t. } & \eta \mathbf{z}^{\mathrm{T}} \mathbf{A} \mathbf{p}-\mathbf{b}^{\mathrm{T}} \mathbf{p}=0, \\
& \mathbf{z}=\mathbf{p}, \\
& p_{m_{\text {m }}} \leq p_{m} \leq p_{m_{\text {max }}}, 1 \leq m \leq M .
\end{aligned}
$$

Then, by introducing an augmented Lagrangian, we have

$$
\begin{aligned}
\max _{\mathbf{e}, \mu} \min _{\mathbf{p}, \mathbf{z}} & \mathbf{1}^{\mathrm{T}} \mathbf{p}+\frac{\rho_{0}}{2}\|\mathbf{p}-\mathbf{z}+\mathbf{e}\|^{2} \\
& +\frac{\rho_{1}}{2}\left\|\eta \mathbf{z}^{\mathrm{T}} \mathbf{A} \mathbf{p}-\mathbf{b}^{\mathrm{T}} \mathbf{p}+\mu\right\|^{2}, \\
\text { s.t. } & p_{m_{\min }} \leq p_{m} \leq p_{m_{\max }}, 1 \leq m \leq M .
\end{aligned}
$$

With the initialization of $\mathbf{p}^{(0)}=\mathbf{z}^{(0)}=\mathbf{p}_{\text {equ }}, \mathbf{e}^{(0)}=\mathbf{1}, \mu^{(0)}=429$ 1 , and $\rho_{0}^{(0)}$ and $\rho_{1}^{(0)}$ set to a large positive number, each iteration 430 involves the following steps.

\section{5}


TABLE I

COMPLEXITY PER ITERATION OF THE OCD-BASED ALGORITHM

\begin{tabular}{|l|r|}
\hline Operation & Flops per iteration \\
\hline Update $\mathbf{p}$ & $3 K M^{2}+(3 K+5) M+3 K$ \\
\hline Update $\lambda$ & $2 K M+2 K+2$, \\
\hline Update $\mathbf{w}$ & $2 K M^{2}+3 K M+5 K$, \\
\hline Update $\boldsymbol{\mu}$ & $2 K M^{2}+K M+4 K$ \\
\hline Total & $7 K M^{2}+(9 K+5) M+14 K+2$ \\
\hline
\end{tabular}

TABLE II

COMPLEXITY PER ITERATION OF THE ADMM-BASED ALGORITHM

\begin{tabular}{|l|r|}
\hline Operation & Flops per iteration \\
\hline \hline Update $\mathbf{p}$ & $M^{3}+(5 K+7) M^{2}+(4 K+8) M+3 K+5$ \\
\hline Update $\mathbf{w}$ & $4 K M^{2}+\left(2 K^{2}+4 K\right) M+K^{2}+14 K$ \\
\hline Update $\mathbf{z}$ & $M^{3}+(7 K+2) M^{2}+(2 K+3) M+4 K$ \\
\hline Update $\mathbf{e}$ & $2 M$ \\
\hline Update $\mu$ & $2 K M+2 K+1$ \\
\hline Update $\gamma$ & $2 K M^{2}+K M+3 K$ \\
\hline Total & $2 M^{3}+(18 K+9) M^{2}+\left(2 K^{2}+13 K+13\right) M$ \\
\hline & $+K^{2}+26 K+6$ \\
\hline
\end{tabular}

- Step 1: Update $\mathbf{p}$. Isolating all the terms involving $\mathbf{p}$, the optimization is a constrained convex problem, leading to

$$
\begin{aligned}
& \overline{\mathbf{p}}^{(n+1)} \\
& \quad=\left(\rho_{0}^{(n)} \mathbf{I}_{M}+\rho_{1}^{(n)}\left(\eta \mathbf{A}^{\mathrm{T}} \mathbf{z}^{(n)}-\mathbf{b}\right)\right. \\
& \left.\quad \times\left(\eta\left(\mathbf{z}^{(n)}\right)^{\mathrm{T}} \mathbf{A}-\mathbf{b}^{\mathrm{T}}\right)\right)^{-1}\left(-\mathbf{1}+\rho_{0}^{(n)}\left(\mathbf{z}^{(n)}-\mathbf{e}^{(n)}\right)\right. \\
& \left.\quad-\rho_{1}^{(n)} \mu^{(n)}\left(\eta \mathbf{A}^{\mathrm{T}} \mathbf{z}^{(n)}-\mathbf{b}\right)\right) \\
& p_{m}^{(n+1)}=\left[\bar{p}_{m}^{(n+1)}\right]_{p_{m_{\text {min }}}}^{p_{m_{\text {max }}}}, 1 \leq m \leq M .
\end{aligned}
$$

- Step 2: Update $\mathbf{z}$. Isolating all the terms involving $\mathbf{z}$, the problem is an unconstrained convex problem, leading to

$$
\begin{aligned}
\mathbf{z}^{(n+1)}= & \left(\rho_{0}^{(n)} \mathbf{I}_{M}+\rho_{1}^{(n)}\left(\eta \mathbf{A} \mathbf{p}^{(n+1)}\right)\left(\eta \mathbf{A} \mathbf{p}^{(n+1)}\right)^{\mathrm{T}}\right)^{-1} \\
& \times\left(\rho_{0}^{(n)}\left(\mathbf{p}^{(n+1)}+\mathbf{e}^{(n)}\right)\right. \\
& \left.+\rho_{1}^{(n)} \eta \mathbf{A} \mathbf{p}^{(n+1)}\left(\mathbf{b}^{\mathrm{T}} \mathbf{p}^{(n+1)}-\mu^{(n)}\right)\right) .
\end{aligned}
$$

- Step 3: Update e and $\mu$. The dual variables are updated according to

$$
\begin{aligned}
& \mu^{(n+1)}=\mu^{(n)}+\eta\left(\mathbf{z}^{(n+1)}\right)^{\mathrm{T}} \mathbf{A} \mathbf{p}^{(n+1)}-\mathbf{b}^{\mathrm{T}} \mathbf{p}^{(n+1)}, \\
& \mathbf{e}^{(n+1)}=\mathbf{e}^{(n)}+\mathbf{p}^{(n+1)}-\mathbf{z}^{(n+1)} .
\end{aligned}
$$

- Step 4: Update the $\rho_{0}$ and $\rho_{1}$ at the first a few iterations. By defining the primal and dual residuals $r_{0}^{(n+1)}$ and $s_{0}^{(n+1)}$ as

$$
\begin{aligned}
& r_{0}^{(n+1)}=\left\|\mathbf{p}^{(n+1)}-\mathbf{z}^{(n+1)}\right\|, \\
& s_{0}^{(n+1)}=\left\|\rho_{0}^{(n)}\left(\mathbf{z}^{(n)}-\mathbf{z}^{(n+1)}\right)\right\|,
\end{aligned}
$$

the updated $\rho_{0}^{(n+1)}$ is given by (75), and the dual variable $\mathbf{e}^{(n+1)}$ is rescaled according to (76). Similarly, define the primal and dual residuals $r_{1}^{(n+1)}$ and $s_{1}^{(n+1)}$ as

441 442 443

$$
\begin{aligned}
r_{1}^{(n+1)}= & \left|\eta\left(\mathbf{z}^{(n+1)}\right)^{\mathrm{T}} \mathbf{A} \mathbf{p}^{(n+1)}-\mathbf{b}^{\mathrm{T}} \mathbf{p}^{(n+1)}\right|, \quad \text { (102) } \\
s_{1}^{(n+1)}= & \| \mu^{(n+1)} \rho_{1}^{(n)} \eta \mathbf{A}^{\mathrm{T}}\left(\mathbf{z}^{(n)}-\mathbf{z}^{(n+1)}\right)+\rho_{1}^{(n)} \eta \\
& \times\left(\eta \mathbf{A}^{\mathrm{T}} \mathbf{z}^{(n)}-\mathbf{b}\right)\left(\mathbf{z}^{(n)}-\mathbf{z}^{(n+1)}\right)^{\mathrm{T}} \mathbf{A} \mathbf{p}^{(n+1)} \| .
\end{aligned}
$$

The updated $\rho_{1}^{(n+1)}$ is given by (77), and the rescaled dual 444 variable $\mu^{(n+1)}$ is given by (78). 445

3) A closed-form approximate solution: An equivalent La- 446 grangian associated with the problem $\mathbb{P} 5$ is $L(\mathbf{p}, \lambda)=\mathbf{1}^{\mathrm{T}} \mathbf{p}+447$ $\lambda\left(\eta \mathbf{p}^{\mathrm{T}} \mathbf{A} \mathbf{p}-\mathbf{b}^{\mathrm{T}} \mathbf{p}\right)$, whose KKT conditions are

$$
\begin{array}{r}
\mathbf{1}+\lambda\left(\eta\left(\mathbf{A}+\mathbf{A}^{\mathrm{T}}\right) \mathbf{p}-\mathbf{b}\right)=\mathbf{0}, \\
\eta \mathbf{p}^{\mathrm{T}} \mathbf{A} \mathbf{p}-\mathbf{b}^{\mathrm{T}} \mathbf{p}=\mathbf{0} .
\end{array}
$$

The authors of [12] obtained the closed-form optimal solution 449 $\lambda^{\star}$ and $\mathbf{p}^{\star}$ by jointly solving the two equations (104) and (105). 450 In particular, they calculated $\overline{\mathbf{p}}^{\star}$ from (104) as

$$
\overline{\mathbf{p}}^{\star}=\frac{\left(\mathbf{A}+\mathbf{A}^{\mathrm{T}}\right)^{-1}}{\eta}\left(\mathbf{b}-\frac{1}{\lambda^{\star}} \mathbf{1}\right),
$$

and then obtained $\mathbf{p}^{\star}$ by imposing the power constraints

$$
p_{m}^{\star}=\left[\bar{p}_{m}^{\star}\right]_{p_{m_{\text {min }}}}^{p_{\text {max }}}, 1 \leq m \leq M .
$$

Unfortunately, this closed-form 'optimal' solution is generally invalid because in general $\mathbf{A}+\mathbf{A}^{\mathrm{T}}$ is not invertible.

Lemma 2: The rank of $\mathbf{A}+\mathbf{A}^{\mathrm{T}}$ is no more than 3 .

Proof:

$$
\begin{aligned}
\operatorname{rank}\left(\mathbf{A}+\mathbf{A}^{\mathrm{T}}\right) \leq & \operatorname{rank}\left(\mathbf{a}_{1,1}\left(\mathbf{a}_{2,2}\right)^{\mathrm{T}}-\mathbf{a}_{1,2}\left(\mathbf{a}_{2,1}\right)^{\mathrm{T}}\right. \\
& \left.+\mathbf{a}_{2,2}\left(\mathbf{a}_{1,1}\right)^{\mathrm{T}}-\mathbf{a}_{2,1}\left(\mathbf{a}_{1,2}\right)^{\mathrm{T}}\right) \\
\leq & \operatorname{rank}\left(\mathbf{a}_{1,1}\left(\mathbf{a}_{2,2}\right)^{\mathrm{T}}\right)+\operatorname{rank}\left(\mathbf{a}_{1,2}\left(\mathbf{a}_{2,1}\right)^{\mathrm{T}}\right) \\
& +\operatorname{rank}\left(\mathbf{a}_{2,2}\left(\mathbf{a}_{1,1}\right)^{\mathrm{T}}\right) \leq 3 .
\end{aligned}
$$

The second inequality is due to the fact that $\mathbf{a}_{1,2}=\mathbf{a}_{2,1}$.

Clearly, for any system with more than 3 transmit radars, the solution of (106) is invalid, and the minimum eigenvalue $\xi_{\min }$ of $\mathbf{A}+\mathbf{A}^{\mathrm{T}}$ is negative. We propose an approximate closed-form solution by replacing the invalid $\left(\mathbf{A}+\mathbf{A}^{\mathrm{T}}\right)^{-1}$ in (106) by the valid regularized form

$$
\mathbf{B}=\left(\mathbf{A}+\mathbf{A}^{\mathrm{T}}+\left(\left|\xi_{\min }\right|+\epsilon\right) \mathbf{I}_{M}\right)^{-1},
$$

where $\epsilon$ is a small positive number, such as, $\epsilon=0.0001$. Thus the 'unconstrained' power allocation is given as

$$
\overline{\mathbf{p}}^{*}=\frac{\mathbf{B}}{\eta}\left(\mathbf{b}-\frac{1}{\lambda^{*}} \mathbf{1}\right),
$$


TABLE III

COMPleXity Per Iteration of the Algorithm Given in [13], Where $n_{\text {in }}$ IS the AVERAGe Number of InNer ItERATIONS IN INNER Optimization PROCEDURE

\begin{tabular}{|l|r|}
\hline Operation & Flops per inner iteration \\
\hline \hline Update the parameters of inner QCLP problem & $\left(5 M^{2}+2 M+1\right) / n_{\text {in }}$ \\
\hline Solve the inner QCLP problem & $M^{3}+(4 K+3) M^{2}+(6 K+10) M-K$ \\
\hline Total & $M^{3}+\left(4 K+3+\frac{5}{n_{\text {in }}}\right) M^{2}+\left(6 K+10+\frac{2}{n_{\text {in }}}\right) M-K$ \\
\hline
\end{tabular}

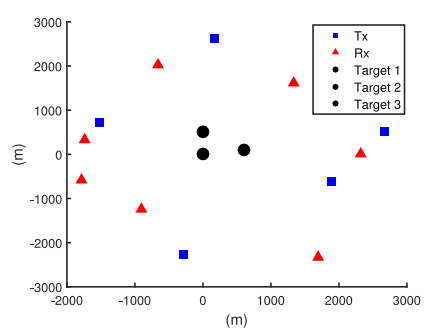

(a)

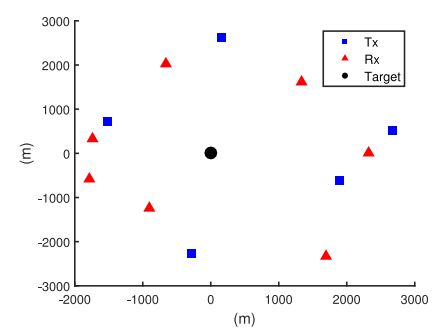

(b)

Fig. 2. Illustration of the MIMO radar system for: (a) three-target application, and (b) single-target application.

where $\lambda^{*}$ is obtained by substituting $\overline{\mathbf{p}}^{*}$ into (105) and taking the positive solution as

$$
\lambda^{*}=\frac{-b+\sqrt{b^{2}-4 a c}}{2 a},
$$

with

$$
\left\{\begin{array}{l}
a=\mathbf{b}^{\mathrm{T}} \mathbf{B}^{\mathrm{T}} \mathbf{A} \mathbf{B} \mathbf{b}-\mathbf{b}^{\mathrm{T}} \mathbf{B} \mathbf{b}, \\
b=-2 \mathbf{1}^{\mathrm{T}} \mathbf{B}^{\mathrm{T}} \mathbf{A}^{\mathrm{T}} \mathbf{B} \mathbf{b}^{\mathrm{T}}+2 \mathbf{b}^{\mathrm{T}} \mathbf{B} \mathbf{1}, \\
c=\mathbf{1}^{\mathrm{T}} \mathbf{B}^{\mathrm{T}} \mathbf{A} \mathbf{B} \mathbf{1}-\mathbf{1}^{\mathrm{T}} \mathbf{B} \mathbf{1} .
\end{array}\right.
$$

The solution $\mathbf{p}^{*}$ is then obtained by projecting $\overline{\mathbf{p}}^{*}$ onto the feasible region. This closed-form solution is inferior to the OCDbased and ADMM-based solutions in terms of its achievable performance, owing to its suboptimal nature.

\section{CONVERGENCE AND COMPLEXITy ANALysis}

Recall from Section II and III that our optimization problem $\mathbb{P} 1$ of (24) is nonconvex, and both our ADMM and OCD algorithms are based on a Lagrangian function approach. It is widely acknowledged that the zero duality gap cannot be guaranteed for general nonconvex problems. However, Yu and Lui [24] proposed a theorem which guarantees the zero duality gap for the nonconvex problem that meets the 'time-sharing condition'. In Appendix B, we proved that our optimization problem $\mathbb{P} 1$ satisfies the time-sharing condition of [24]. Hence, the strong duality holds for $\mathbb{P} 1$. We are now ready to prove that both our two algorithms can converge to a local optimal point under some assumptions.

\section{A. Convergence of the Proposed Algorithms}

1) The ADMM-based algorithm: We first point out again that since our problem is nonconvex, the ADMM-based algorithm can only guarantee to converge to a local optimal solution. The convergence of the ADMM method is proved for the convex optimization problem in [18], while Magnússon et al. 490 [25] extended the convergence results to the nonconvex case. 491 The convergence of our ADMM-based algorithm will be fur- 492 ther illustrated in Section V using simulations.

2) The OCD-based algorithm: Again, since our optimization problem is nonconvex, the OCD-based algorithm can only find a locally optimal solution. Collect all the primal variables of the Lagrangian (32) together as $\mathbf{y}=\left[\mathbf{p}^{\mathrm{T}} \mathbf{w}^{\mathrm{T}}\right]^{\mathrm{T}}$ and denote the cost function and the constraints of $\mathbb{P} 3$ respectively by

$$
\begin{aligned}
f(\mathbf{y}) & =\mathbf{1}^{\mathrm{T}} \mathbf{p}, \\
g_{0}(\mathbf{y}) & =\sum_{k=1}^{K} v_{k} w_{k} \mathbf{b}_{k}^{\mathrm{T}} \mathbf{p}-1, \\
g_{k}(\mathbf{y}) & =w_{k} \eta \mathbf{p}^{\mathrm{T}} \mathbf{A}_{k} \mathbf{p}-1,1 \leq k \leq K .
\end{aligned}
$$

According to Theorem 2 in Section 8.2.3 and Lemma 5 in 499 Section 2.1 of [26], to prove the convergence of the OCD al- 500 gorithm, we have to prove that the second derivatives $\nabla^{2} f(\mathbf{y}) \quad 501$ and $\nabla^{2} g_{k}(\mathbf{y})$ for $0 \leq k \leq K$ satisfy the Lipschitz condition in 502 a neighbourhood of the optimal primal point $\mathbf{y}^{\star}$. Note that 503

$$
\begin{aligned}
& \nabla^{2} f(\mathbf{y})=\mathbf{0}, \\
& \nabla^{2} g_{0}(\mathbf{y})=\left[\begin{array}{cc}
\mathbf{0} & v_{1} \mathbf{b}_{1} \cdots v_{K} \mathbf{b}_{K} \\
v_{1} \mathbf{b}_{1}^{\mathrm{T}} & \\
\vdots & \mathbf{0} \\
v_{K} \mathbf{b}_{K}^{\mathrm{T}} &
\end{array}\right] \text {, }
\end{aligned}
$$

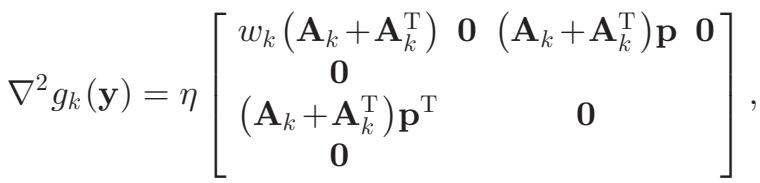

$$
\begin{aligned}
& 1 \leq k \leq K .
\end{aligned}
$$

Since $\nabla^{2} f(\mathbf{y})$ and $\nabla^{2} g_{0}(\mathbf{y})$ are constants, they satisfy the 504 required Lipschitz condition. For $\mathbf{p}_{\min } \leq \mathbf{p} \leq \mathbf{p}_{\max }$, all the el- 505 ements in the matrix $\nabla^{2} g_{k}(\mathbf{y})$, where $1 \leq k \leq K$, are finite. 506 Therefore, it is easy to find a constant $\varsigma$ satisfying

$$
\left\|\nabla^{2} g_{k}\left(\mathbf{y}_{1}\right)-\nabla^{2} g_{k}\left(\mathbf{y}_{2}\right)\right\| \leq \varsigma\left\|\mathbf{y}_{1}-\mathbf{y}_{2}\right\| .
$$

Thus $\nabla^{2} g_{k}(\mathbf{y})$ satisfies the required Lipschitz condition too. $\quad 508$

According to [26], under the assumption that the Hessian ma- 509 trix of the Lagrangian (32) with respect to $y$ at the minimum pri- 510 mal point $\mathbf{y}^{\star}=\left(\mathbf{p}^{\star}, \mathbf{w}^{\star}\right)$ is positive definite, the Hessian matrix 511 of the Lagrangian (32) with respect to the primal and dual vari- 512 ables is negative definite at the optimal point $\left(\mathbf{p}^{\star}, \mathbf{w}^{\star}, \lambda^{\star}, \boldsymbol{\mu}^{\star}\right) .513$ Then there exists a positive number $\bar{\kappa}=\min _{i}-\Re\left[\bar{\xi}_{i}\right]\left|\bar{\xi}_{i}\right|^{-2}, \quad 514$ 
TABLE IV

SYSTEM PARAMETERS

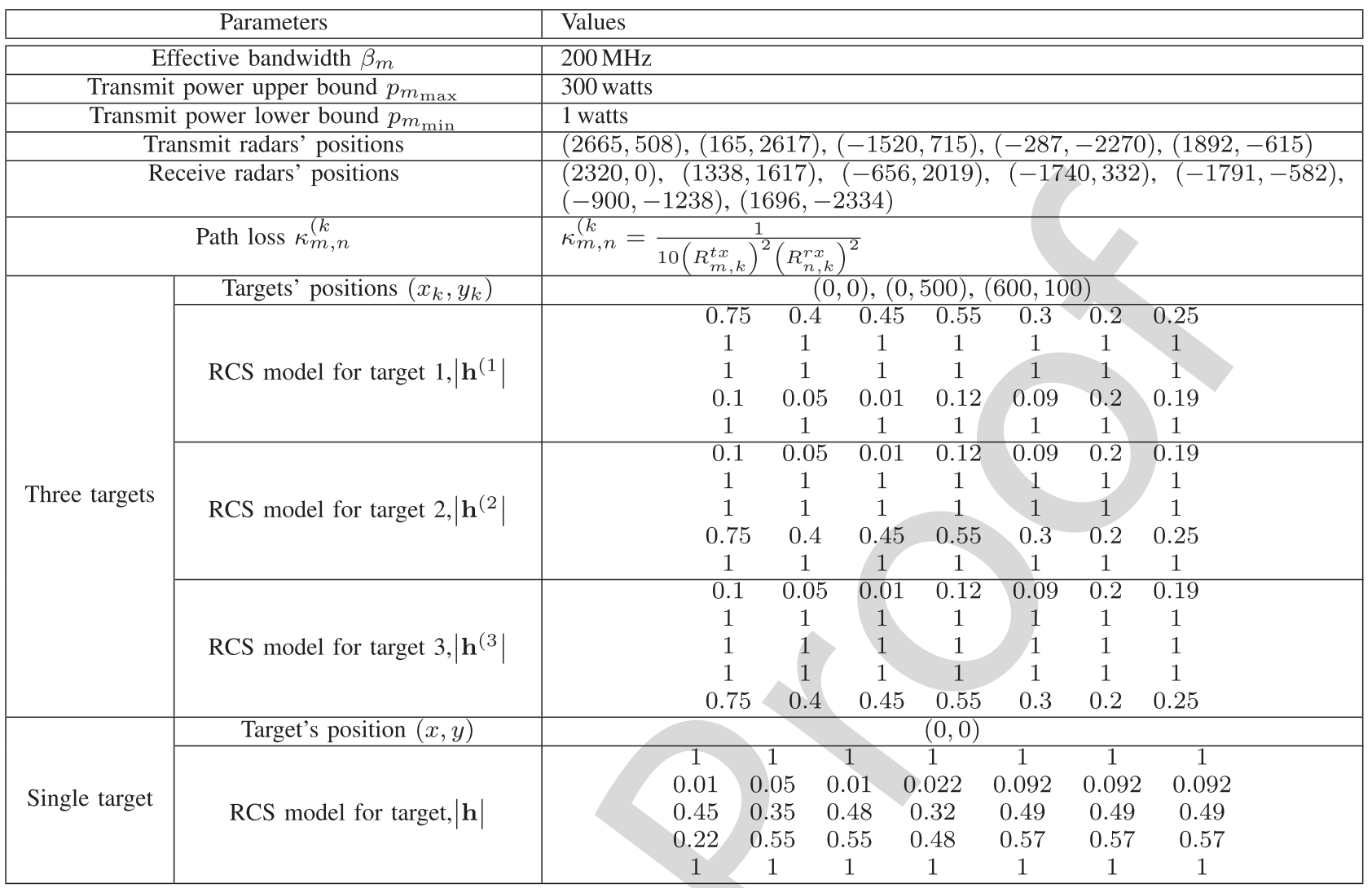

where $\bar{\xi}_{i}$ are the eigenvalues of the Hessian matrix of the Lagrangian (32) with respect to the primal and dual variables at $\left(\mathbf{p}^{\star}, \mathbf{w}^{\star}, \lambda^{\star}, \boldsymbol{\mu}^{\star}\right)$. Consequently, as long as the maximum of the four step sizes $\kappa_{\max }=\max _{1 \leq i \leq 4} \kappa_{i}$ satisfies the condition of $\kappa_{\max } \leq \bar{\kappa}$, our scheme (37)-(40) will converge to the locally optimal point $\left(\mathbf{p}^{\star}, \mathbf{w}^{\star}, \lambda^{\star}, \boldsymbol{\mu}^{\star}\right)$ when starting from a neighbourhood of $\left(\mathbf{p}^{\star}, \mathbf{w}^{\star}, \lambda^{\star}, \boldsymbol{\mu}^{\star}\right)$, according to [26]. In practice, $\bar{\kappa}$ is unknown. It is advisable to choose sufficiently small step sizes $\kappa_{i}, 1 \leq i \leq 4$, in order to ensure the convergence of the OCD scheme.

Remark 4: A positive-definite Hessian matrix of the Lagrangian (32) with respect to $\mathbf{y}$ at $\mathbf{y}^{\star}$ is a sufficient condition for the convergence of the OCD scheme. If this Hessian matrix is semi-positive definite, we cannot prove the convergence of the OCD scheme based on the result of [26]. By adopting an exponentially decaying step size $\kappa_{\max } \propto e^{-\alpha n}$, we ensure that our OCD algorithm works well in any situation.

\section{B. Complexity of Proposed Algorithms and Algorithm of [13]}

The complexity of our OCD and ADMM algorithms are summarized in Tables I and II, respectively. For the ADMM-based algorithm, since the penalty parameters are only updated in the first few iterations, the complexity associated with this part of operation is omitted. Additionally, we assume that GaussJordan elimination is used for matrix inversion and, therefore, the number of flops required by inverting an $M \times M$ matrix is $M^{3}+M^{2}+M$. For the OCD-based algorithm, the complexity of computing the four step sizes is negligible and therefore it 541 is also omitted. Clearly, the complexity of the ADMM-based 542 algorithm is on the order of $M^{3}$ per iteration, which is denoted 543 by $\mathrm{O}\left(M^{3}\right)$, while the complexity of the OCD-based algorithm 544 is on the order of $\mathrm{O}\left(M^{2}\right)$ per iteration. It will be shown by our 545 simulation results that the convergence speed of the ADMM al- 546 gorithm is at least one order of magnitude faster than that of the 547 OCD algorithm. Therefore, despite its higher per-iteration com- 548 plexity, the ADMM algorithm actually imposes a lower total 549 complexity, compared to the OCD algorithm.

The benchmark scheme of [13] invokes two iterative loops for solving the optimization problem (25). Specifically, at each outer iteration, the parameters of the inner quadratic constrained linear programming (QCLP) problem are updated, and the QCLP problem is then solved iteratively in the inner iterative loop. We assume that the interior-point method is used for solving this inner QCLP, which requires $n_{\text {in }}$ iterations on average. Based on the above discussions, the complexity of the algorithm of [13] is summarized in Table III, where it is seen that the complexity per inner iteration is on the order of $\mathrm{O}\left(M^{3}\right)$. Thus the complexity of our ADMM-based algorithm is only marginally higher than that of the algorithm in [13], because they are both on the order of $\mathrm{O}\left(M^{3}\right)$ per iteration. The algorithm of [13] requires a total of $n_{\mathrm{ou}} n_{\mathrm{in}}$ iterations to converge, where $n_{\mathrm{ou}}$ is the number of iterations for the outer iterative loop. As it will be shown in the simulation results, the number of iterations required for the ADMM-based algorithm to converge is very close to the total number of iterations $n_{\mathrm{ou}} n_{\text {in }}$ required by the algorithm of [13]. 


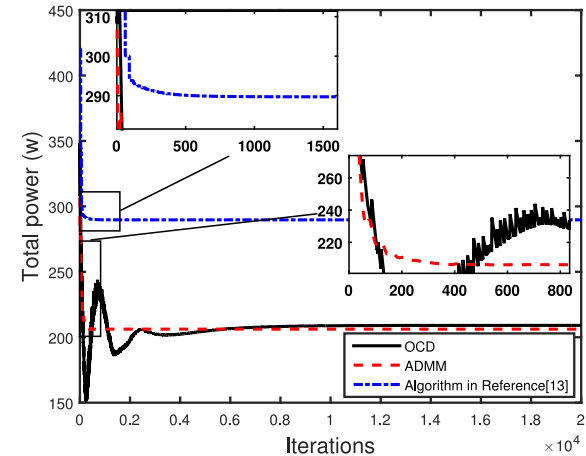

(a)

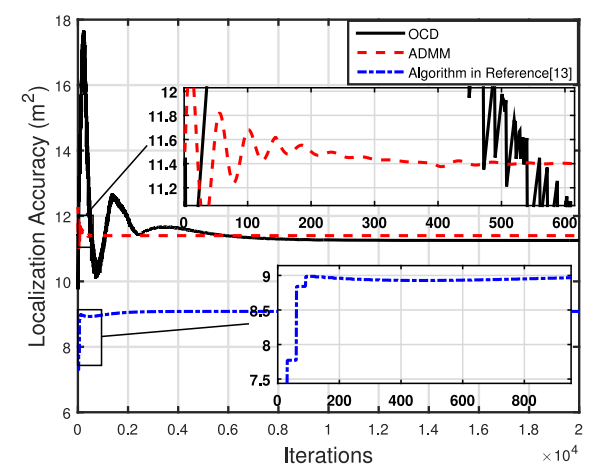

(b)

Fig. 3. Convergence performance of three algorithms, in terms of (a) total power consumption, and (b) aggregate localization accuracy, for the three-target case with $v_{1}=1, v_{2}=2$ and $v_{3}=1$.

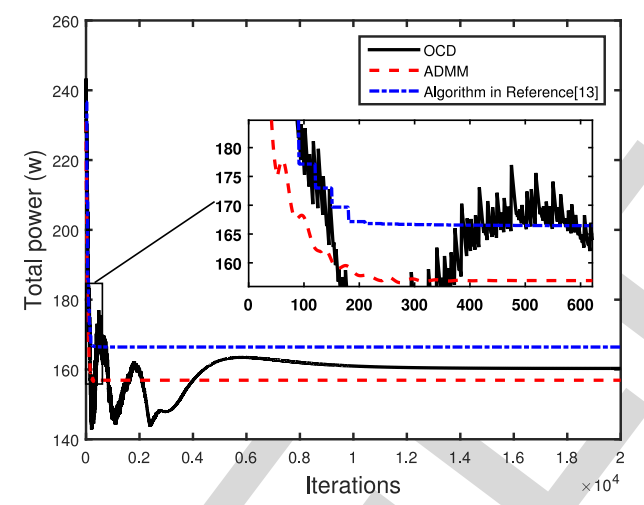

(a)

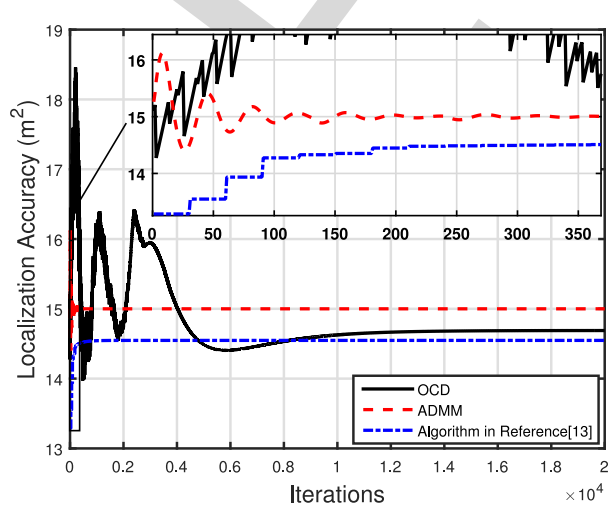

(b)

Fig. 4. Convergence performance of three algorithms, in terms of (a) total power consumption, and (b) aggregate localization accuracy, for the three-target case with $v_{1}=v_{2}=v_{3}=1$.
In this sense, both algorithms require a similar total complexity 569 for solving their associated optimization problems. Although 570 our OCD-based algorithm enjoys a much lower complexity per 571 iteration than the algorithm of [13], it imposes a higher total 572 complexity.

\section{Simulation Results}

Let us now evaluate the performance of the proposed al- 575 gorithms using a MIMO radar system having $M=5$ trans- 576 mit radars and $N=7$ receive radars. The algorithm of [13] is 577 used as the benchmark. Fig. 2 depicts both the triple-target and 578 single-target cases considered. The system parameters of both 579 the triple-target and single-target cases are listed in Table IV. The 580 localization accuracy threshold $\eta$ is set to $15 \mathrm{~m}^{2}$ for the triple- 581 target case and $10 \mathrm{~m}^{2}$ for the single-target case. The exponential 582 decaying factor is empirically chosen to be $\alpha=0.0005$ for the 583 four step sizes of the OCD algorithm.

\section{A. Triple-Target Case}

We consider the two sets of the importance weightings for the three targets given by: i) $v_{1}=1, v_{2}=2$ and $v_{3}=1$, and ii) $v_{1}=v_{2}=v_{3}=1$. For the sake of a fair comparison to the algorithm of [13], the effects of these weightings have to be taken into consideration, and the target estimation error thresholds for the three constraints of the optimization problem (25) are suitably scaled as

$$
\frac{\mathbf{b}_{1}^{\mathrm{T}} \mathbf{p}}{\mathbf{p}^{\mathrm{T}} \mathbf{A}_{1} \mathbf{p}} \leq \bar{\eta}_{1}, \frac{\mathbf{b}_{2}^{\mathrm{T}} \mathbf{p}}{\mathbf{p}^{\mathrm{T}} \mathbf{A}_{2} \mathbf{p}} \leq \bar{\eta}_{2}, \frac{\mathbf{b}_{3}^{\mathrm{T}} \mathbf{p}}{\mathbf{p}^{\mathrm{T}} \mathbf{A}_{3} \mathbf{p}} \leq \bar{\eta}_{3},
$$

with $\bar{\eta}_{1}=\frac{1}{3 v_{1}} \eta, \bar{\eta}_{2}=\frac{1}{3 v_{2}} \eta$ and $\bar{\eta}_{3}=\frac{1}{3 v_{3}} \eta$. For our ADMM algorithm, the initial values of the dual variables are set to $\mathbf{e}^{(0)}=\left[\begin{array}{llll}1 & 1 & 1 & 1\end{array}\right]^{\mathrm{T}}, \mu^{(0)}=1$ and $\gamma_{k}^{(0)}=1$ for $1 \leq k \leq 3$, while all the initial penalty parameters are set to 500 . For our OCD algorithm, the initial values of the dual variables are set to $\lambda^{(0)}=1$ and $\mu_{k}^{(0)}=1$ for $1 \leq k \leq 3$. Additionally, the four constants in the four step sizes of the OCD algorithm are set to $c_{1}=0.3, c_{2}=1.0, c_{3}=1.5$ and $c_{4}=1.1$ for the senario i), while they are set to $c_{1}=0.3, c_{2}=0.9, c_{3}=1.5$ and $c_{4}=1.1$ for the senario ii). These parameters were found empirically to be appropriate for the corresponding application scenarios. For the algorithm of [13], we use the CVX software to solve its inner QCLP problem. In our simulations, we observe that the CVX converges within 25 to 35 iterations. Therefore, we will assume that the average number of inner iterations for the algorithm of [13] is $n_{\text {in }}=30$.

Fig. 3 compares the total power allocations $\mathrm{p}$ and the aggregate localization accuracy results of $\sum_{k=1}^{3} \frac{\mathbf{b}_{k}^{\mathrm{T}} \mathbf{p}}{\mathbf{p}^{\mathrm{T}} \mathbf{A}_{k} \mathbf{p}}$ obtained by the three algorithms for the senario i), while Fig. 4 depicts the results for the senario ii). It can be seen that the number of iterations required by the ADMM-based algorithm to converge is similar to the total number of iterations $n_{\text {ou }} n_{\text {in }}$ required by the algorithm of [13], while the convergence speed of the OCDbased algorithm is considerably slower than that of the other two algorithms. As expected, our algorithms outperform the algorithm of [13] in terms of its total power consumption, albeit at the expense of some degradation in localization accuracy. 587 588 589 590 591 592

\section{5} 616 617 618 619 (n) , . (as 
TABLE V

PERFormance COMPARISON OF THREE AlgORITHMS FOR THE THREE-TARGET CASE

\begin{tabular}{|c|c|c|c|c|c|c|}
\hline & \multicolumn{3}{|c|}{ ii) $v_{1}=v_{2}=v_{3}=1$} & \multicolumn{3}{|c|}{ i) $v_{1}=1, v_{2}=2, v_{3}=1$} \\
\hline & ADMM & OCD & {$[13]$} & ADMM & OCD & {$[13]$} \\
\hline Radar 1: Power (watts) & 1 & 1 & 1 & 1 & 1 & 1 \\
\hline Radar 2: Power (watts) & 95.8 & 93.3 & 102 & 119.6 & 117.9 & 75.8 \\
\hline Radar 3: Power (watts) & 58.2 & 64.0 & 40.3 & 83.5 & 88.1 & 170.4 \\
\hline Radar 4: Power (watts) & 1 & 1 & 1 & 1 & 1 & 1 \\
\hline Radar 5: Power (watts) & 1 & 1 & 22.2 & 1 & 1 & 41.5 \\
\hline Total Power (watts) & 157 & 160.3 & 166.5 & 206.1 & 209.0 & 289.7 \\
\hline Target 1: Localization Accuracy $\left(\mathrm{m}^{2}\right)$ & 5.4 & 5.3 & 5 & 4.1 & 4.1 & 3.1 \\
\hline Target 2: Localization Accuracy $\left(\mathrm{m}^{2}\right)$ & 4.8 & 4.7 & 4.5 & 3.6 & 3.5 & 2.5 \\
\hline Target 3: Localization Accuracy $\left(\mathrm{m}^{2}\right)$ & 4.8 & 4.7 & 5 & 3.7 & 3.6 & 3.5 \\
\hline Aggregate Localization Accuracy $\left(\mathrm{m}^{2}\right)$ & 15 & 14.7 & 14.5 & 11.4 & 11.3 & 9.1 \\
\hline Total Power Saving & $5.7 \%$ & $3.7 \%$ & - & $28.9 \%$ & $27.9 \%$ & - \\
\hline Degradation in Aggregate Localization Accuracy & $3.4 \%$ & $1.4 \%$ & - & $25.3 \%$ & $27.9 \%$ & \\
\hline Average Total Power Saving & $10.0 \%$ & $10.5 \%$ & 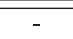 & $20.0 \%$ & $25.6 \%$ & 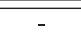 \\
\hline Average Degradation in Aggregate Localization Accuracy & $8.6 \%$ & $8.9 \%$ & - & $27.2 \%$ & $30.0 \%$ & - \\
\hline
\end{tabular}

The average results are obtained over 1000 random simulation experiments.

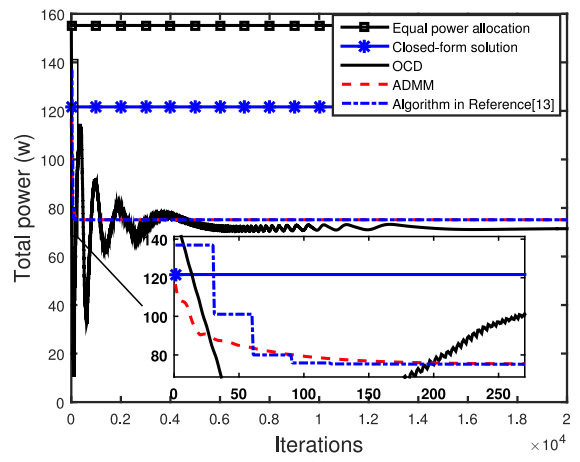

(a)

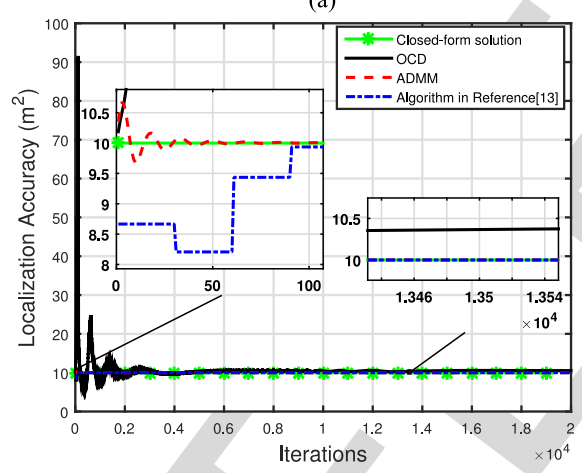

(b)

Fig. 5. Convergence performance of three algorithms, in terms of (a) total power consumption, and (b) aggregate localization accuracy, in comparison with the EPA and the closed-form solution, for the single-target case.

Table $\mathrm{V}$ details how our algorithms trade the localization accuracy against the transmit power, in comparison to the algorithm of [13]. Specifically, for the senario of i), our ADMM algorithm achieves $28.9 \%$ power saving at the cost of $25.3 \%$ degradation in aggregate localization accuracy, while our OCD algorithm trades $27.9 \%$ power saving against $27.9 \%$ degradation in localization accuracy. For the equal weighting senario of ii), the savings in power achieved by our two algorithms are considerably smaller but the losses in localization accuracy are also significantly smaller, compared with the senario i). To obtain statistically relevant comparison, we carry out 1000 simulations by randomly locating all the transmit radars and receive radars at 631 the radius $R=3000\left(0.5+\varepsilon_{x}\right)$ m with the angular rotations of 632 $\theta=2 \pi \varepsilon_{y}$, where $\varepsilon_{x}$ and $\varepsilon_{y}$ are uniformly distributed in $[0,1.0] .633$ The average power saving and degradation in localization accu- 634 racy over the 1000 random experiments are listed in the last two 635 rows of Table $\mathrm{V}$.

\section{B. Single-Target Case}

The four constants in the four step sizes of the OCD algorithm are set to $c_{1}=c_{2}=1.0$ and $c_{3}=c_{4}=0.3$, which is empirically found to be appropriate for this application senario. Fig. 5 characterizes the performance of our ADMM-based and OCD-based algorithms as well as the algorithm of [13]. As expected, all the three algorithms attain the same performance, both in terms of total power allocated and localization accuracy, since the underlying optimization problems are identical in the single-target case. In terms of convergence speed, our ADMM-based algorithm outperforms the algorithm of [13] while the OCD-based algorithm is considerably slower than the algorithm of [13]. In Fig. 5 (a), we also characterize the equalpower allocation (EPA) scheme and the closed-form solution of SubSection III-B3. It can be seen that our closed-form solution performs significantly better than the EPA scheme, but it is inferior to the other three iterative algorithms because the suboptimal nature of this closed-form solution.

\section{CONCLUSION}

The target localization problem of distributed MIMO radar systems has been investigated, which minimizes the power of the transmit radars, while meeting a required localization accuracy. We have proposed the OCD-based and ADMM-based iterative algorithms to solve this nonconvex optimization problem. Both the algorithms are capable of converging to a local optimum. The OCD algorithm imposes a much lower computational complexity per iteration, while the ADMM algorithm achieves a much faster convergence. For the multi-target senario, we have shown how our proposed approach trades the power saving with some degradation in localization accuracy, 
compared with that of state-of-the-art scheme [13]. We have also demonstrated that our ADMM-based algorithm and the existing state-of-the-art scheme have similar computational complexity and convergence speed. For the single-target senario, we have confirmed that our algorithms and the benchmark attain the same performance in terms of both power consumption and localization accuracy, because the underlying optimization problems become identical.

\section{A. Derivation of Updating Formulae for Penalty Parameters}

The optimal solution to the $\mathbb{P} 4$ of (45) should be primal and dual feasible, that is,

$$
\begin{aligned}
& \mathbf{p}^{(n+1)}-\mathbf{z}^{(n+1)}=\mathbf{0}, \\
& \sum_{k=1}^{K} w_{k}^{(n+1)} v_{k} \mathbf{b}_{k}^{\mathrm{T}} \mathbf{p}^{(n+1)}-1=0, \\
& w_{k}\left(\mathbf{z}^{(n+1)}\right)^{\mathrm{T}} \mathbf{A}_{k} \mathbf{p}^{(n+1)} \eta-1=0,1 \leq k \leq K, \\
& \frac{\partial L^{\prime}\left(\mathbf{p}, \mathbf{z}^{(n+1)}, \mathbf{w}^{(n+1)}, \mathbf{d}_{0}^{(n+1)}, d_{1}^{(n+1)}, \mathbf{d}_{2}^{(n+1)}\right)}{\partial \mathbf{p}}=\mathbf{0}, \\
& \frac{\partial L^{\prime}\left(\mathbf{p}^{(n+1)}, \mathbf{z}^{(n+1)}, \mathbf{w}, \mathbf{d}_{0}^{(n+1)}, d_{1}^{(n+1)}, \mathbf{d}_{2}^{(n+1)}\right)}{\partial \mathbf{w}}=\mathbf{0}, \\
& \frac{\partial L^{\prime}\left(\mathbf{p}^{(n+1)}, \mathbf{z}, \mathbf{w}^{(n+1)}, \mathbf{d}_{0}^{(n+1)}, d_{1}^{(n+1)}, \mathbf{d}_{2}^{(n+1)}\right)}{\partial \mathbf{z}}=\mathbf{0},
\end{aligned}
$$

where $L^{\prime}\left(\mathbf{p}, \mathbf{w}, \mathbf{z}, \mathbf{d}_{0}, d_{1}, \mathbf{d}_{2}\right)$ is the Lagrangian of (45), which can be separated into three parts

$$
\begin{aligned}
& L^{\prime}\left(\mathbf{p}, \mathbf{w}, \mathbf{z}, \mathbf{d}_{0}, d_{1}, \mathbf{d}_{2}\right)=\underbrace{\mathbf{1}^{\mathrm{T}} \mathbf{p}+\mathbf{d}_{0}^{\mathrm{T}}(\mathbf{p}-\mathbf{z})}_{L_{0}^{\prime}\left(\mathbf{p}, \mathbf{z}, \mathbf{d}_{0}\right)}+ \\
& \underbrace{d_{1}\left(\sum_{k=1}^{K} w_{k} v_{k} \mathbf{b}_{k}^{\mathrm{T}} \mathbf{p}-1\right)}_{L_{1}^{\prime}\left(\mathbf{p}, \mathbf{w}, d_{1}\right)}+\underbrace{\sum_{k=1}^{K} d_{2, k}\left(w_{k} \mathbf{z}^{\mathrm{T}} \mathbf{A}_{k} \mathbf{p} \eta-1\right)}_{L_{2}^{\prime}\left(\mathbf{p}, \mathbf{w}, \mathbf{z}, \mathbf{d}_{2}\right)} .
\end{aligned}
$$

However, the ADMM-based algorithm uses the augmented Lagrangian of

$$
\begin{gathered}
L\left(\mathbf{p}, \mathbf{w}, \mathbf{z}, \mathbf{d}_{0}, d_{1}, \mathbf{d}_{2}\right)=\underbrace{\mathbf{1}^{\mathrm{T}} \mathbf{p}+\frac{\rho_{0}}{2}\|\mathbf{p}-\mathbf{z}\|^{2}+\mathbf{d}_{0}^{\mathrm{T}}(\mathbf{p}-\mathbf{z})}_{L_{0}\left(\mathbf{p}, \mathbf{z}, \mathbf{d}_{0}\right)} \\
+\underbrace{\frac{\rho_{1}}{2}\left|\sum_{k=1}^{K} w_{k} v_{k} \mathbf{b}_{k}^{\mathrm{T}} \mathbf{p}-1\right|^{2}+d_{1}\left(\sum_{k=1}^{K} w_{k} v_{k} \mathbf{b}_{k}^{\mathrm{T}} \mathbf{p}-1\right)}_{L_{1}\left(\mathbf{p}, \mathbf{w}, d_{1}\right)} \\
+\underbrace{\sum_{k=1}^{K} \frac{\rho_{2, k}}{2}\left|w_{k} \mathbf{z}^{\mathrm{T}} \mathbf{A}_{k} \mathbf{p} \eta-1\right|^{2}+\sum_{k=1}^{K} d_{2, k}\left(w_{k} \mathbf{z}^{\mathrm{T}} \mathbf{A}_{k} \mathbf{p} \eta-1\right)}_{L_{2}\left(\mathbf{p}, \mathbf{w}, \mathbf{z}, \mathbf{d}_{2}\right)},
\end{gathered}
$$

which can be divided into three parts, and all the primal and 683 dual variables are updated one by one. Thus, in every iteration, 684 there exist primal and dual residuals.

Specifically, in the $(n+1)$ th iteration, the primal residuals 686 are given by $r_{0}^{(n+1)}$ of (65), $r_{1}^{(n+1)}$ of (66), and $r_{2, k}^{(n+1)}$ for 687 $1 \leq k \leq K$ of (67), while the dual residuals are defined via

$$
d r=\sqrt{\left\|\mathbf{d r}_{0}\right\|^{2}+\left\|\mathbf{d r}_{1}\right\|^{2}+\left\|\mathbf{d r}_{2}\right\|^{2}},
$$

with

$$
\begin{aligned}
\mathbf{d r}_{0}= & \frac{\partial L\left(\mathbf{p}, \mathbf{z}^{(n)}, \mathbf{w}^{(n)}, \mathbf{d}_{0}^{(n)}, d_{1}^{(n)}, \mathbf{d}_{2}^{(n)}\right)}{\partial \mathbf{p}} \\
& -\frac{\partial L^{\prime}\left(\mathbf{p}, \mathbf{z}^{(n+1)}, \mathbf{w}^{(n+1)}, \mathbf{d}_{0}^{(n+1)}, d_{1}^{(n+1)}, \mathbf{d}_{2}^{(n+1)}\right)}{\partial \mathbf{p}}, \\
\mathbf{d r}_{1}= & \frac{\partial L\left(\mathbf{p}^{(n+1)}, \mathbf{z}^{(n)}, \mathbf{w}, \mathbf{d}_{0}^{(n)}, d_{1}^{(n)}, \mathbf{d}_{2}^{(n)}\right)}{\partial \mathbf{w}} \\
& -\frac{\partial L^{\prime}\left(\mathbf{p}^{(n+1)}, \mathbf{z}^{(n+1)}, \mathbf{w}, \mathbf{d}_{0}^{(n+1)}, d_{1}^{(n+1)}, \mathbf{d}_{2}^{(n+1)}\right)}{\partial \mathbf{w}}, \\
\mathbf{d r}_{2}= & \frac{\partial L\left(\mathbf{p}^{(n+1)}, \mathbf{z}, \mathbf{w}^{(n+1)}, \mathbf{d}_{0}^{(n)}, d_{1}^{(n)}, \mathbf{d}_{2}^{(n)}\right)}{\partial \mathbf{z}} \\
& -\frac{\partial L^{\prime}\left(\mathbf{p}^{(n+1)}, \mathbf{z}, \mathbf{w}^{(n+1)}, \mathbf{d}_{0}^{(n+1)}, d_{1}^{(n+1)}, \mathbf{d}_{2}^{(n+1)}\right)}{\partial \mathbf{z}} .
\end{aligned}
$$

It can be seen that the primal residuals $r_{0}^{(n+1)}, r_{1}^{(n+1)}$ and 690 $r_{2, k}^{(n+)}$ for $1 \leq k \leq K$ are related to $L_{0}\left(\mathbf{p}, \mathbf{z}, \mathbf{d}_{0}\right), L_{1}\left(\mathbf{p}, \mathbf{w}, d_{1}\right) 691$ and $L_{2}\left(\mathbf{p}, \mathbf{w}, \mathbf{z}, \mathbf{d}_{2}\right)$, respectively. Therefore, we will similarly 692 'separate' the dual residuals into $s_{0}^{(n+1)}, s_{1}^{(n+1)}$ and $s_{2, k}^{(n+1)}$ for 693 $1 \leq k \leq K$, corresponding to $L_{0}\left(\mathbf{p}, \mathbf{z}, \mathbf{d}_{0}\right), L_{1}\left(\mathbf{p}, \mathbf{w}, d_{1}\right)$ and 694 $L_{2}\left(\mathbf{p}, \mathbf{w}, \mathbf{z}, \mathbf{d}_{2}\right)$, respectively.

In order to analyze the updating formula (75) for the penalty 696 parameter $\rho_{0}$, we have to calculate $s_{0}^{(n+1)}$ as follows

$$
\begin{aligned}
s_{0}^{(n+1)}= & \left(\left\|\frac{\partial L_{0}\left(\mathbf{p}^{(n+1)}, \mathbf{z}, \mathbf{d}_{0}^{(n)}\right)}{\partial \mathbf{z}}-\frac{\partial L_{0}^{\prime}\left(\mathbf{p}^{(n+1)}, \mathbf{z}, \mathbf{d}_{0}^{(n+1)}\right)}{\partial \mathbf{z}}\right\|^{2}\right. \\
& \left.+\left\|\frac{\partial L_{0}\left(\mathbf{p}, \mathbf{z}^{(n)}, \mathbf{d}_{0}^{(n)}\right)}{\partial \mathbf{p}}-\frac{\partial L_{0}^{\prime}\left(\mathbf{p}, \mathbf{z}^{(n+1)}, \mathbf{d}_{0}^{(n+1)}\right)}{\partial \mathbf{p}}\right\|^{2}\right)^{\frac{1}{2}} .
\end{aligned}
$$

By evaluating the required four partial derivatives and plugging 698 them into (131), we arrive at the dual residual $s_{0}^{(n+1)}$ of (68). 699 Note that a large value for $\rho_{0}$ adds a large penalty on the violation 700 of primal feasibility and, therefore, a large $\rho_{0}$ reduces the primal 701 residual $r_{0}^{(n+1)}$. On the other hand, from the expression (68), it 702 is seen that a small $\rho_{0}$ reduces the dual residual $s_{0}^{(n+1)}$. Thus, 703 in order to balance the primal and dual residuals $r_{0}^{(n+1)}$ and 704 $s_{0}^{(n+1)}$, the penalty parameter $\rho_{0}$ is updated according to (75), 705 which is beneficial to convergence.

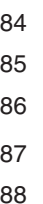


707 Similarly, it can be shown that the dual residual $s_{1}^{(n+1)}$ re708 lated to $L_{1}\left(\mathbf{p}, \mathbf{w}, d_{1}\right)$ is given by (69) and (71), while the dual 709 residuals $s_{2, k}^{(n+1)}$ for $1 \leq k \leq K$ related to $L_{2}\left(\mathbf{p}, \mathbf{w}, \mathbf{z}, \mathbf{d}_{2}\right)$ are 710 specified by (70), (72) and (73). Following the same logic of 711 balancing the primal and dual residuals, the updating formulae 712 for the penalty parameters $\rho_{1}$ and $\rho_{2, k}$ are specified by (76) and 713 (78), respectively.

\section{4}

\section{B. Proof of the Time-Sharing Condition for Problem $\mathbb{P} 1$}

According to [24], the time-sharing condition for the optimization problem $\mathbb{P} 1$ of (24) is as follows. Time-sharing condition: Let $\mathbf{p}_{1}$ and $\mathbf{p}_{2}$ be the optimal solutions of $\mathbb{P} 1$ in conjunction with $\eta=\eta_{1}$ and $\eta=\eta_{2}$, respectively. $\mathbb{P} 1$ is said to satisfy the time-sharing condition if for any $\eta_{1}$ and $\eta_{2}$ and for any $0 \leq \xi \leq 1$, there always exists a feasible solution $\mathbf{p}_{3}$ so that $\sum_{k=1}^{K} v_{k} \frac{\mathbf{b}_{k}^{\mathrm{T}} \mathbf{p}_{3}}{\mathbf{p}_{3}^{\mathrm{T}} \mathbf{A}_{k} \mathbf{p}_{3}} \leq \xi \eta_{1}+(1-\xi) \eta_{2}$ and $\mathbf{1}^{\mathrm{T}} \mathbf{p}_{3} \geq$ $\xi \mathbf{1}^{\mathrm{T}} \mathbf{p}_{1}+(1-\xi) \mathbf{1}^{\mathrm{T}} \mathbf{p}_{2}$.

According to Lemma 1 , if we set $\mathbf{p}_{3}=\mathbf{p}_{\max }$, then

$$
\sum_{k=1}^{K} v_{k} \frac{\mathbf{b}_{k}^{\mathrm{T}} \mathbf{p}_{3}}{\mathbf{p}_{3}^{\mathrm{T}} \mathbf{A}_{k} \mathbf{p}_{3}} \leq \eta_{1} \text { and } \sum_{k=1}^{K} v_{k} \frac{\mathbf{b}_{k}^{\mathrm{T}} \mathbf{p}_{3}}{\mathbf{p}_{3}^{\mathrm{T}} \mathbf{A}_{k} \mathbf{p}_{3}} \leq \eta_{2} .
$$

$$
\begin{aligned}
& \sum_{k=1}^{K} v_{k} \frac{\mathbf{b}_{k}^{\mathrm{T}} \mathbf{p}_{3}}{\mathbf{p}_{3}^{\mathrm{T}} \mathbf{A}_{k} \mathbf{p}_{3}}=\xi \sum_{k=1}^{K} v_{k} \frac{\mathbf{b}_{k}^{\mathrm{T}} \mathbf{p}_{3}}{\mathbf{p}_{3}^{\mathrm{T}} \mathbf{A}_{k} \mathbf{p}_{3}} \\
& \quad+(1-\xi) \sum_{k=1}^{K} v_{k} \frac{\mathbf{b}_{k}^{\mathrm{T}} \mathbf{p}_{3}}{\mathbf{p}_{3}^{\mathrm{T}} \mathbf{A}_{k} \mathbf{p}_{3}} \leq \xi \eta_{1}+(1-\xi) \eta_{2}, \\
& \mathbf{1}^{\mathrm{T}} \mathbf{p}_{3}=\xi \mathbf{1}^{\mathrm{T}} \mathbf{p}_{3}+(1-\xi) \mathbf{1}^{\mathrm{T}} \mathbf{p}_{3} \geq \xi \mathbf{1}^{\mathrm{T}} \mathbf{p}_{1}+(1-\xi) \mathbf{1}^{\mathrm{T}} \mathbf{p}_{2} .
\end{aligned}
$$

Therefore, $\mathbb{P} 1$ satisfies the time-sharing condition and the dual gap for our nonconvex problem is zero.

\section{REFERENCES}

[1] J. Li and P. Stoica, MIMO Radar Signal Processing. Hoboken, NJ, USA: Wiley, 2009.

[2] Y. Yu, A. P. Petropulu, and H. V. Poor, "Measurement matrix design for compressive sensing-based MIMO radar," IEEE Trans. Signal Process., vol. 59, no. 11, pp. 5338-5352, Nov. 2011.

[3] S. Gogineni and A. Nehorai, "Target estimation using sparse modeling for distributed MIMO radar," IEEE Trans. Signal Process., vol. 59, no. 11, pp. 5315-5325, Nov. 2011.

[4] Y. Yao, A. P. Petrppulu, and H. V. Poor, "CSSF MIMO radar: Compressivesensing and step-frequency based MIMO radar," IEEE Trans. Aerosp. Electron. Syst., vol. 48, no. 2, pp. 1490-1504, Apr. 2012.

[5] J. Xu, X.-Z. Dai, X.-G. Xia, L.-B. Wang, J. Yu, and Y.-N. Peng, "Optimization of multisite radar system with MIMO radars for target detection," IEEE Trans. Aerosp. Electron. Syst., vol. 47, no. 4, pp. 2329-2343, Oct. 2011.

[6] E. Fishler, A. Haimovich, R. S. Blum, L. J. Cimini, D. Chizhik, and R. A. Valenzuela, "Spatial diversity in radars-models and detection performance," IEEE Trans. Signal Process., vol. 54, no. 3, pp. 823-838, Mar. 2006.

[7] Q. He, N. H. Lehmann, R. S. Blum, and A. M. Haimovich, "MIMO-radar moving target detection in homogeneous clutter," IEEE Trans. Aerosp. Electron. Syst., vol. 46, no. 3, pp. 1290-1301, Jul. 2010.

[8] Q. He, R. S. Blum, H. Godrich, and A. M. Haimovich, "Cramer-Rao bound for target velocity estimation in MIMO radar with widely separated antennas," in Proc. 42nd Annu. Conf. Inf. Sci. Syst., Mar. 19-21, 2008, pp. 123-127.

[9] H. Godrich, A. M. Haimovich, and R. S. Blum, "Target localisation techniques and tools for multiple-input multiple-output radar," IET Radar, Sonar Navigat., vol. 3, no. 4, pp. 314-327, Aug. 2009.

[10] Q. He, R. S. Blum, H. Godrich, and A. M. Haimovich, "Target velocity estimation and antenna placement for MIMO radar with widely separated antennas," IEEE J. Sel. Topics Signal Process., vol. 4, no. 1, pp. 79-100, Feb. 2010.

[11] H. Godrich, A. M. Haimovich, and R. S. Blum, "Target localization accuracy gain in MIMO radar-based systems," IEEE Trans. Inf. Theory, vol. 56, no. 6, pp. 2783-2803, Jun. 2010.

[12] H. Godrich, A. P. Petropulu, and H. V. Poor, "Power allocation strategies for target localization in distributed multiple-radar architectures," IEEE Trans. Signal Process., vol. 59, no. 7, pp. 3226-3240, Jul. 2011.

[13] N. Garcia, A. M. Haimovich, M. Coulon, and M. Lops, "Resource allocation in MIMO radar with multiple targets for non-coherent localization," IEEE Trans. Signal Process., vol. 62, no. 10, pp. 2656-2666, May 2014.

[14] N. H. Lehmann et al., "Evaluation of transmit diversity in MIMO-radar direction finding," IEEE Trans. Signal Process., vol. 55, no. 5, pp. 2215 2225, May 2007.

[15] C. Wei, Q. He, and R. S. Blum, "Cramer-Rao bound for joint location and velocity estimation in multi-target non-coherent MIMO radars," in Proc. 44th Аnnu. Conf. Inf. Sci. Syst., Mar. 17-19, 2010, pp. 1-6.

[16] Q. He and R. S. Blum, "CramerRao bound for MIMO radar target localization with phase errors," IEEE Signal Process. Lett., vol. 17, no. 1, pp. 83-86, Jan. 2010.

[17] A. J. Conejo, E. Castillo, R. Minguez, and R. Garcia-Bertrand, Decomposition Techniques in Mathematical Programming: Engineering and Science Applications. Berlin, Germany: Springer-Verlag, 2006.

[18] S. Boyd, N. Parikh, E. Chu, B. Peleato, and J. Eckstein, "Distributed optimization and statistical learning via the alternating direction method of multipliers," Found. Trends Mach. Learn., vol. 3, no. 1, pp. 1-122, 2011.

[19] A. Simonetto and G. Leus, "Distributed maximum likelihood sensor network localization," IEEE Trans. Signal Process., vol. 62, no. 6, pp. 1424 1437, Mar. 2014.

[20] S. M. Kay, Fundamentals of Statistical Signal Processing, Volume II: Detection Theory. Upper Saddle River, NJ, USA: Prentice-Hall, 1998.

[21] H. Godrich, A. M. Haimovich, and R. S. Blum, "Cramer Rao bound on target localization estimation in MIMO radar systems," in Proc. 42nd Annu. Conf. Inf. Sci. Syst., Mar. 19-21, 2008, pp. 134-139.

[22] H. Godrich, A. P. Petropulu, and H. V. Poor, "Sensor selection in distributed multiple-radar architectures for localization: A knapsack problem formulation," IEEE Trans. Signal Process., vol. 60, no. 1, pp. 247-260, Jan. 2012.

[23] F. Yousefian, A. Nedić, and U. V. Shanbhag, "On stochastic gradient an subgradient methods with adaptive steplength sequences," Automatica, vol. 48, no. 1, pp. 56-67, Jan. 2012.

[24] W. Yu and R. Lui, "Dual methods for nonconvex spectrum optimization of multicarrier systems," IEEE Trans. Commun., vol. 54, no. 7, pp. 13101322, Jul. 2006

[25] S. Magnússon, P. C. Weeraddana, M. G. Rabbat, and C. Fischione, "On the convergence of alternating direction Lagrangian methods for nonconvex structured optimization problems," IEEE Trans. Control Netw. Syst., vol. PP, no. 99, pp. 1-14, doi: 10.1109/TCNS.2015.2476198, preprint.

[26] B. T. Polyak, Introduction to Optimization. New York, NY, USA: Optimization Software, Inc., 1987.

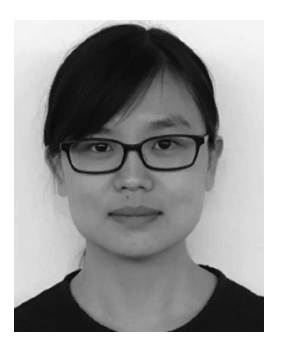

Ying Ma received the B.E. degree in electronic engineering from the Changchun Institute of Technology, Jin Lin, China, in 2011. She is currently working toward the Ph.D. degree with the Research Institute of Communication Technology, Beijing Institute of Technology, Beijing, China. Since July 2015, she has been with the Department of Electrical and Computer Engineering, University of Southampton, Southampton, U.K., where she is a visiting Ph.D. student under the supervision of Prof. L. Hanzo and Prof. S. Chen. Her research interests include distributed computation, multiple-input multiple-output systems, and cooperative communication.
752 753 754 755 756 757 758 759 760 761 762 763 764 765 766 767 768 769 770 771 772 773 774 775 776 777 778 779 780 781 782 783 784 785 786 787 788 789 790 791 792 793 794 795 796 797 798 799 800 801 802 803 804 805 806 


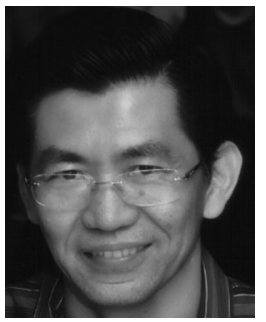

Sheng Chen (M'90-SM'97-F'08) received the B.Eng. degree in control engineering from the East China Petroleum Institute, Dongying, China, in January 1982, the Ph.D. degree in control engineering from the City University, London, U.K., in September 1986, and the D.Sc. degree from the University of Southampton, Southampton, U.K., in 2005. He held research and academic appointments with the University of Sheffield, the University of Edinburgh, and the University of Portsmouth, U.K., from 1986 to 1999 . Since 1999 , he has been with the Electronics and Computer Science Department, University of Southampton, where he is a Professor of intelligent systems and signal processing. His research interests include adaptive signal processing, wireless communications, modeling and identification of nonlinear systems, neural network and machine learning, intelligent control system design, evolutionary computation methods, and optimization. He has authored more than 550 research papers. He is a Fellow of the United Kingdom Royal Academy of Engineering, a Fellow of the IET, and a Distinguished Adjunct Professor with King Abdulaziz University, Jeddah, Saudi Arabia. He was an ISI Highly Cited Researcher in engineering in 2004.

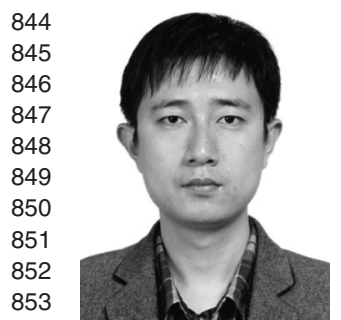

Chengwen Xing (S'08-M'10) received the B.Eng. degree from Xidian University, Xi' an, China, in 2005 , and the Ph.D. degree from the University of Hong Kong, Hong Kong, in 2010. Since September 2010, he has been with the School of Information and Electronics, Beijing Institute of Technology, Beijing, China, where he is currently an Associate Professor From September 2012 to December 2012, he was a Visiting Scholar at the University of Macau. His current research interests include statistical signal processing, convex optimization, multivariate statistics,

855 combinatorial optimization, massive MIMO systems, and high-frequency band 856 communication systems. He is currently serving as an Associate Editor for the 857 IEEE TRANSACTIONS ON VEHICULAR TECHNOLOGY, KSII Transactions on In858 ternet and Information Systems, Transactions on Emerging Telecommunications 859 Technologies, and China Communications.

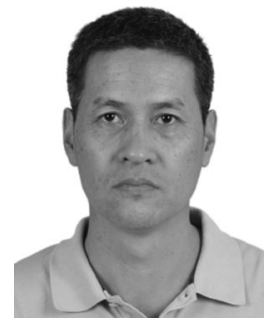

Xiangyuan Bu received the B.Eng., Master's, and 861 $\mathrm{Ph} . \mathrm{D}$. degrees from the Beijing Institute of Technol- 862 ogy, Beijing, China, in 1987, 1993, and 2007, re- 863 spectively. He is currently a Professor in the Wireless 864 Communications and Networks Laboratory, School 865 of Information and Electronics, Beijing Institute of 866 Technology. From July 1987 to September 1990, he 867 was a Researcher in the Institute of Harbin, 674 Fac- 868 tory, and from April 1993 to May 2002, he was a Re- 869 searcher in the No. 23 Institute of China Aerospace 870 Science and Industry. His current research interests 871 include wireless communication and signal processing.
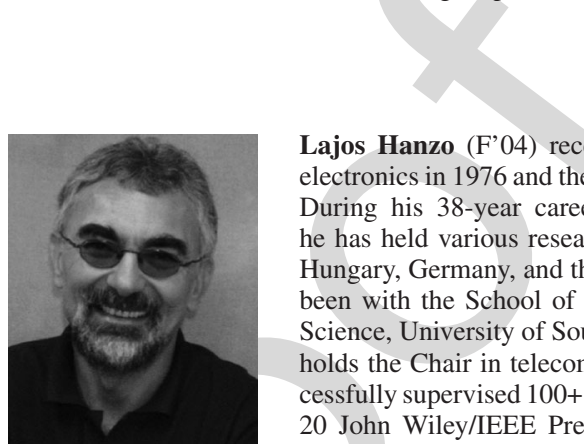

Lajos Hanzo (F'04) received the D.Sc. degree in 874 electronics in 1976 and the Doctorate degree in 1983. 875 During his 38-year career in telecommunications, 876 he has held various research and academic posts in 877 Hungary, Germany, and the U.K. Since 1986, he has 878 been with the School of Electronics and Computer 879 Science, University of Southampton, U.K., where he 880 holds the Chair in telecommunications. He has suc- 881 cessfully supervised $100+\mathrm{Ph}$. D. students, coauthored 882 20 John Wiley/IEEE Press books on mobile radio 883 communications, totalling in excess of 10000 pages, 884 published 1590 research entries at IEEE Xplore, acted both as the TPC Chair 885 and the General Chair of IEEE conferences, presented keynote lectures, and 886 has received a number of distinctions. He is currently directing a 60-strong 887 academic research team, working on a range of research projects in the field of 888 wireless multimedia communications sponsored by industry, the Engineering 889 and Physical Sciences Research Council, U.K., the European Research Coun- 890 cil's Advanced Fellow Grant, and the Royal Society's Wolfson Research Merit 891 Award. He is an enthusiastic supporter of industrial and academic liaison and he 892 offers a range of industrial courses. He is also a Governor of the IEEE VTS. In 893 2009, he received the honorary doctorate "Doctor Honoris Causa" by the Tech- 894 nical University of Budapest. During 2008-2012, he was the Editor-in-Chief 895 of the IEEE Press and also a Chaired Professor with Tsinghua University, Bei- 896 jing, China. His research is funded by the European Research Council's Senior 897 Research Fellow Grant. He is a Fellow of the Royal Academy of Engineering, 898 IET, and EURASIP. For further information on research in progress and asso- 899 ciated publications, please refer to http://www-mobile.ecs.soton.ac.uk. He has 900 $25000+$ citations. 873

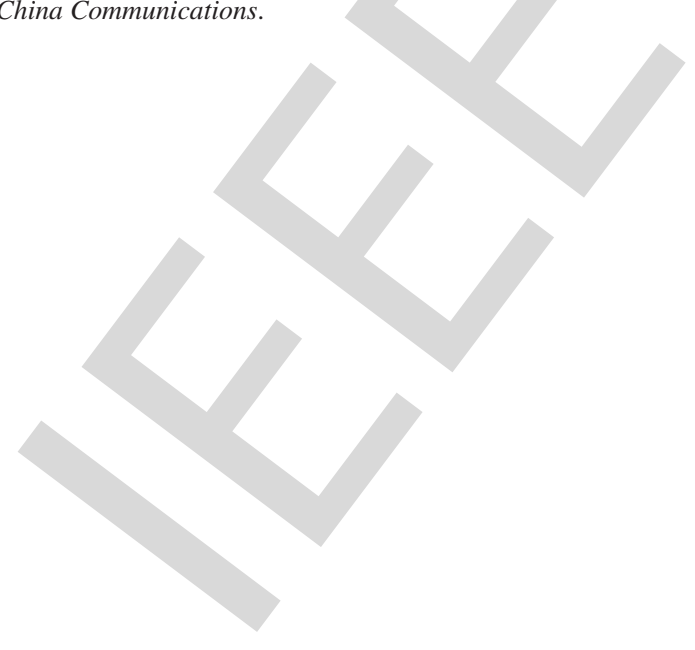


Q1. Author: "Scenario" is spelled as "senario.” Please check.

904

Q2. Author: Please provide full bibliographic details in Ref. [25]. 
Ying Ma, Sheng Chen, Fellow, IEEE, Chengwen Xing, Member, IEEE, Xiangyuan Bu, and Lajos Hanzo, Fellow, IEEE

Abstract-Distributed radar systems are capable of enhancing the detection performance by using multiple widely spaced distributed antennas. With prior statistic information of targets, resource allocation is of critical importance for further improving the system's achievable performance. In this paper, the total transmitted power is minimized at a given mean-square target-estimation error. We derive two iterative decomposition algorithms for solving this nonconvex constrained optimization problem, namely, the optimality condition decomposition (OCD)-based and the alternating direction method of multipliers (ADMM)-based algorithms. Both the convergence performance and the computational complexity of our algorithms are analyzed theoretically, which are then confirmed by our simulation results. The OCD method imposes a much lower computational burden per iteration, while the ADMM method exhibits a higher per-iteration complexity, but as a benefit of its significantly faster convergence speed, it requires less iterations. Therefore, the ADMM imposes a lower total complexity than the OCD. The results also show that both of our schemes outperform the state-of-the-art benchmark scheme for the multiple-target case, in terms of the total power allocated, at the cost of some degradation in localization accuracy. For the single-target case, all the three algorithms achieve similar performance. Our ADMM algorithm has similar total computational complexity per iteration and convergence speed to those of the benchmark.
\end{abstract}

Index Terms-Alternating direction method of multipliers, localization, multiple-input multiple-output radar, optimality condition decomposition, resource allocation.

\section{INTRODUCTION}

$\mathbf{M}$ ULTIPLE-input multi-output (MIMO) radar systems relying on widely-separated antennas have attracted considerable attention from both industry and academia. The family of distributed MIMO radar systems is capable of significantly improving the estimation/detection performance [1]-[6] by exploiting the increased degrees of freedom resulting from the improved spatial diversity. In particular, distributed radar systems are capable of improving accuracy of target location and

Manuscript received March 8, 2016; revised June 17, 2016 and July 18, 2016; accepted August 9, 2016. The associate editor coordinating the review of this manuscript and approving it for publication was Dr. Fauzia Ahmad. This work was supported in part by the National Natural Science Foundation of China under Grants 61421001 and 61671058.

Y. Ma, C. Xing, and X. Bu are with the School of Information and Electronics, Beijing Institute of Technology, Beijing 100081, China (e-mail: mayingbit2011@gmail.com; xingchengwen@gmail.com; bxy@bit.edu.cn).

$\mathrm{S}$. Chen is with the School of Electronics and Computer Science, University of Southampton, Southampton SO17 1BJ, U.K., and also with King Abdulaziz University, Jeddah 21589, Saudi Arabia (e-mail: sqc@ecs.soton.ac.uk).

L. Hanzo is with the School of Electronics and Computer Science, University of Southampton, Southampton SO17 1BJ, U.K. (e-mail: lh@ecs.soton.ac.uk).

Color versions of one or more of the figures in this paper are available online at http://ieeexplore.ieee.org.

Digital Object Identifier 10.1109/TSP.2016.2602801

\section{Decomposition Optimization Algorithms for Distributed Radar Systems}

velocity estimation by exploiting the different Doppler estimates from multiple spatial directions [7]-[10].

Naturally, the localization performance of MIMO radar systems relying on widely-spaced distributed antennas, quantified in terms of the mean square estimation error (MSE), is determined by diverse factors, including effective signal bandwidth, the signal-to-noise ratio (SNR), the product of the numbers of transmit and receive antennas, etc [11]. Since the SNR is influenced by the path loss, the target radar cross section (RCS) and the transmitted power, the attainable localization performance can be improved by increasing either the number of participating radars or the transmitted power. However, simply increasing the amount of resources without considering the cooperation among the individual terminals is usually far from optimal.

In most traditional designs, the system's power budget is usually allocated to the transmit radars and it is fixed [6], [10], which is easy to implement and results in the simplest network structure. However, when prior estimation of the target RCS is available, according to estimation theory, uniform power allocation is far from the best strategy. In battlefields, a radar system is usually supported by power-supply trucks, but under hostile environments, their number is strictly limited. Thus, how to allocate limited resources to multiple radar stations is of great importance for maximizing the achievable performance. In other words, power allocation substantially affects the detection performance of multi-radar systems.

Recently, various studies used the Cramer-Rao lower bound (CRLB) for evaluating the performance of MIMO radar systems [11]-[16]. A power allocation scheme [12] based on CRLB was designed for multiple radar systems with a single target. The resultant nonconvex optimization problem was solved by relaxation and a domain-decomposition method. Specifically, in [12] the total transmitted power was minimized at a given estimation MSE threshold. However the algorithm of [12] was not designed for multiple-target scenarios, which are often encountered in practice. In [13] a power allocation algorithm was proposed for the multiple-target case, which is equally applicable to the single-target senario.

Against this background, in this paper, we propose two iterative decomposition methods, which are referred to as the optimality condition decomposition (OCD) [17] and the alternating direction method of multipliers (ADMM) [18] algorithms, in order to minimize the total transmitted power while satisfying a predefined estimation MSE threshold. These two algorithms can be applied to both multiple-target and single-target scenarios. The ADMM method has been widely adopted for solving convex problems. In this paper, we extend the ADMM algorithm to nonconvex problems and show that it is capable of converging. 
It is worth pointing out that Simonetto and Leus [19] applied the ADMM method to solve a localization problem in a sensor network by converting the nonconvex problem to a convex one using rank-relaxation. However, the algorithm of [19] cannot be applied to our problem, because the task of [19] is that of locating sensors, which is not directly related to the signal waveform and power. Furthermore, the maximum likelihood (ML) criterion can be used for solving this sensor localization problem. However, our task is to assign the power of every MIMO radar transmitter, and at the time of writing it is an open challenge to design the ML estimator for this task [11]. The main contributions of our work are as follows.

- We propose two iterative decomposition algorithms, namely, the OCD-based and ADMM-based methods, for both multiple-target and single-target scenarios. The convergence of these two algorithms is analyzed theoretically and verified by simulations. Both these two methods are capable of converging to locally optimal solutions. The complexity analysis of the two algorithms is provided and it is shown that the OCD method imposes a much lower computational burden per iteration, while the ADMM method enjoys a significantly faster convergence speed and therefore it actually imposes a lower total complexity.

- In the multiple-target case, we demonstrate that both of our two algorithms outperform the state-of-the-art benchmark scheme of [13], in terms of the total power allocated at the expense of some degradation in localization accuracy. We show furthermore that our ADMM-based algorithm and the algorithm of [13] have similar convergence speed and total computational complexity.

- In the single-target case, we show that all the three methods attain a similar performance, since the underlying optimization problems are identical. We also prove that the closed-form solution of [12] is invalid for the systems with more than three transmit radars and we propose a beneficial suboptimal closed-form solution.

The paper is organized as follows. In Section II, the MIMO radar system model is introduced and the corresponding optimization problem is formulated. Our power allocation strategies are proposed in Section III for both the multiple-target and single-target cases, while our convergence and complexity analysis is provided in Section IV. Section V presents our simulation results for characterizing the attainable performance of the proposed algorithms which are then compared to the scheme of [13]. Finally, our conclusions are offered in Section VI.

Throughout our discussions, the following notational conventions are used. Boldface lower- and upper-case letters denote vectors and matrices, respectively. The transpose, conjugate and inverse operators are denoted by $(\cdot)^{\mathrm{T}},(\cdot)^{*}$ and $(\cdot)^{-1}$, respectively, while $\operatorname{Tr}(\cdot)$ stands for the matrix trace operation and $\operatorname{diag}\left(x_{1}, x_{2}, \cdots, x_{n}\right)$ or $\operatorname{diag}(\mathbf{x})$ is the diagonal matrix with the specified diagonal elements. Additionally, $\operatorname{diag}\left(\mathbf{X}_{1}, \cdots, \mathbf{X}_{K}\right)$ and $\operatorname{diag}\left(\mathbf{x}_{1}, \cdots, \mathbf{x}_{K}\right)$ denotes the block diagonal matrices with the specified sub-matrices and vectors, respectively, at the corresponding block diagonal positions. The operator $\mathrm{v}_{\text {diag }}(\mathbf{X})$ forms a vector using the diagonal elements of square matrix $\mathbf{X}$, while $\mathrm{E}\{\cdot\}$ denotes the expectation operator and $\otimes$ is the

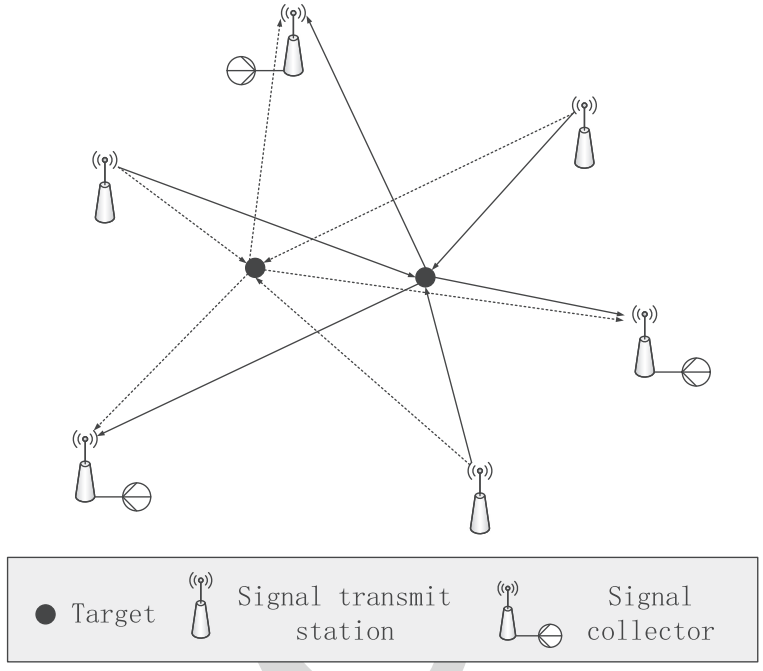

Fig. 1. Illustration of distributed radar network.

Kronecker product operator. The sub-matrix consisting of the 145 elements of the $i_{1}$ to $i_{2}$ rows and $j_{1}$ to $j_{2}$ columns of $\mathbf{A}$ is 146 denoted by $[\mathbf{A}]_{\left[i_{1}: i_{2} ; j_{1}: j_{2}\right]}$, and the $i$ th row and $j$ th column ele- 147 ment of $\mathbf{A}$ is given by $[\mathbf{A}]_{i, j}$. Similarly, $[\mathbf{a}]_{\left[i_{1}: i_{2}\right]}$ is the vector 148 consisting of $i_{1}$ th to $i_{2}$ th elements of $\mathbf{a}$. The magnitude operator 149 is given by $|\cdot|$, and $\|\cdot\|$ denotes the vector two-norm or matrix 150 Frobenius norm. $\mathbf{I}_{K}$ is the identity matrix of size $K \times K$ and $\mathbf{0} 151$ is the zero matrix/vector of an appropriate size, while $\mathbf{1}$ denotes 152 the vector of an appropriate size, whose elements are all equal 153 to one. Finally, $\Re[]$ denotes the real part of a complex value and 154 $\mathrm{j}=\sqrt{-1}$ represents the imaginary axis.

\section{SYSTEM MODEL}

The MIMO radar system consists of $M$ transmit radars and $N$ receive radars which cooperate to locate $K$ targets, as illustrated in Fig. 1. The $M$ transmit radars are positioned at the coordinates $\left(x_{m}^{t x}, y_{m}^{t x}\right)$ for $1 \leq m \leq M$, and the $N$ receive radars are positioned at $\left(x_{n}^{r x}, y_{n}^{r x}\right)$ for $1 \leq n \leq N$, while the position of target $k$ is $\left(x_{k}, y_{k}\right)$. A set of mutually orthogonal waveforms are transmitted from the transmit radars, and the corresponding baseband signals are denoted by $\left\{s_{m}(t)\right\}_{m=1}^{M}$ with normalized power, i.e., $\int_{\tau_{m}}\left|s_{m}(t)\right|^{2} d t=1$, where $\tau_{m}$ is the duration of the $m$ th transmitted signal. Furthermore, the orthogonality of the transmitted waveforms can always be guaranteed even for different time delays, i.e., $\int_{\tau_{m}} s_{m}(t) s_{m^{\prime}}^{*}(t-\tau) d t=0$ for $m^{\prime} \neq m$. The narrowband signals of the transmitted waveforms have the effective bandwidth $\beta_{m}$ specified by

$$
\beta_{m}^{2}=\frac{\int_{W} f^{2}\left|S_{m}(f)\right|^{2} d f}{\int_{W}\left|S_{m}(f)\right|^{2} d f}(\mathrm{~Hz})^{2}
$$

where $W$ is the frequency range of the signals, and $S_{m}(f)$ is the Fourier transform of $s_{m}(t)$ transmitted from the $m$ th transmit radar. The transmitted powers of the different antennas, denoted by $\mathbf{p}=\left[p_{1} p_{2} \cdots p_{M}\right]^{\mathrm{T}}$, are constrained by their corresponding 
minimum and maximum values specified by

$$
\begin{aligned}
& \mathbf{p}_{\text {min }}=\left[p_{1_{\text {min }}} p_{2_{\text {min }}} \cdots p_{M_{\text {min }}}\right]^{\mathrm{T}}, \\
& \mathbf{p}_{\max }=\left[p_{1_{\text {max }}} p_{2_{\text {max }}} \cdots p_{M_{\text {max }}}\right]^{\mathrm{T}} .
\end{aligned}
$$

176

202
The upper bound $p_{m_{\max }}$ is determined by the design and the lower bound $p_{m_{\min }}$ is used to guarantee that the transmit radar $m$ operates at an appropriate SNR. Let the propagation path spanning from the transmitter $m$ to the target $k$ and from the target $k$ to the receiver $n$ be defined as the channel $(m, k, n)$. Then the propagation time $\tau_{m, n}^{(k}$ of the channel $(m, k, n)$ can be calculated by $\tau_{m, n}^{(k}=\left(R_{m, k}^{t x}+R_{n, k}^{r x}\right) / c$, where $c$ is the speed of light, $R_{m, k}^{t x}=\sqrt{\left(x_{m}^{t x}-x_{k}\right)^{2}+\left(y_{m}^{t x}-y_{k}\right)^{2}}$ is the distance from transmitter $m$ to target $k$, and $R_{n, k}^{r x}=$ $\sqrt{\left(x_{n}^{r x}-x_{k}\right)^{2}+\left(y_{n}^{r x}-y_{k}\right)^{2}}$ is the distance from target $k$ to receiver $n$. The time delay $\tau_{m, n}^{(k}$ is used to estimate the position of targets. For far field signals, by retaining only the linear terms of its Taylor expansion, $\tau_{m, n}^{(k}$ can be approximated as a linear function of $x_{k}$ and $y_{k}$

$$
\begin{aligned}
\tau_{m, n}^{(k} \simeq & -\frac{x_{k}}{c}\left(\cos \theta_{m}^{(k}+\cos \varphi_{n}^{(k}\right) \\
& -\frac{y_{k}}{c}\left(\sin \theta_{m}^{(k}+\sin \varphi_{n}^{(k)},\right.
\end{aligned}
$$

where $\theta_{m}^{(k}$ is the bearing angle of the transmitting radar $m$ to the target $k$ and $\varphi_{n}^{(k}$ is the bearing angle of the receiving radar $n$ to the target $k$, both measured with respect to the $x$ axis.

Let the complex-valued reflectivity coefficient $h_{m, n}^{(k}$ represent the attenuation and phase rotation of channel $(m, k, n)$. The baseband signal at receive radar $n$ can be expressed as

$$
r_{n}(t)=\sum_{k=1}^{K} \sum_{m=1}^{M} \sqrt{p_{m}} h_{m, n}^{(k} s_{m}\left(t-\tau_{m, n}^{(k}\right)+\omega_{n}(t),
$$

where $\omega_{n}(t)$ is a circularly symmetric complex Gaussian white noise, which is bandlimited to the system bandwidth $W$ and hence has a zero mean and $E\left\{\left|\omega_{n}(t)\right|^{2}\right\}=\sigma^{2}$. In our work, the path-loss $\kappa_{m, n}^{(k}$ is chosen as

$$
\kappa_{m, n}^{(k} \propto \frac{1}{\left(R_{m, k}^{t x}\right)^{2}\left(R_{n, k}^{r x}\right)^{2}} .
$$

Thus, given the complex target RCS $\zeta_{m, n}^{(k}$, the channel coefficient $h_{m, n}^{(k}$ is given by

$$
h_{m, n}^{(k}=\zeta_{m, n}^{(k} \sqrt{\kappa_{m, n}^{(k}}=h_{m, n}^{(k, \mathrm{Re}}+\mathrm{j} h_{m, n}^{(k, \mathrm{Im}},
$$

where $h_{m, n}^{(k, \operatorname{Re}}$ and $h_{m, n}^{(k, \mathrm{Im}}$ are the real and imaginary parts of $h_{m, n}^{(k}$. Let us collect all the channel coefficients associated with the target $k$ in the $(2 M N \times 1)$-element real-valued vector as

$$
\mathbf{h}_{k}=\left[h_{1,1}^{(k, \operatorname{Re}} \cdots h_{1, N}^{(k, R e} \cdots h_{M, N}^{(k, \operatorname{Re}} h_{1,1}^{(k, \operatorname{Im}} \cdots h_{1, N}^{(k, \operatorname{Im}} \cdots h_{M, N}^{(k, \operatorname{Im}}\right]^{\mathrm{T}} .
$$

Similarly, we introduce the $(N M \times 1)$-element real vectors

$$
\begin{aligned}
\left|\mathbf{h}^{(k}\right|^{2} & =\left[\left|h_{1,1}^{(k}\right|^{2} \cdots\left|h_{1, N}^{(k}\right|^{2} \cdots\left|h_{M, 1}^{(k}\right|^{2} \cdots\left|h_{M, N}^{(k}\right|^{2}\right]^{\mathrm{T}}, \\
\left|\mathbf{h}^{(k}\right| & =\left[\left|h_{1,1}^{(k}\right| \cdots\left|h_{1, N}^{(k}\right| \cdots\left|h_{M, 1}^{(k}\right| \cdots\left|h_{M, N}^{(k}\right|\right]^{\mathrm{T}} .
\end{aligned}
$$

Upon defining $\mathbf{h}=\left[\mathbf{h}_{1}^{\mathrm{T}} \mathbf{h}_{2}^{\mathrm{T}} \cdots \mathbf{h}_{K}^{\mathrm{T}}\right]^{\mathrm{T}}$ and the location vector 206 of the $K$ targets as $\boldsymbol{l}_{x, y}=\left[x_{1} y_{1} \cdots x_{K} y_{K}\right]^{\mathrm{T}}$, all the system's 207 parameters can be stacked into a single real-valued vector

$$
\mathbf{u}=\left[\boldsymbol{l}_{x, y}^{\mathrm{T}} \mathbf{h}^{\mathrm{T}}\right]^{\mathrm{T}} .
$$

Since the received signal (5) is also a function of the time delays 209 $\tau_{m, n}^{(k}$, we also define the following system parameter vector

$$
\boldsymbol{\psi}=\left[\tau_{1,1}^{(1} \cdots \tau_{1, N}^{(1} \cdots \tau_{M, N}^{(K} \mathbf{h}^{\mathrm{T}}\right]^{\mathrm{T}} .
$$

There exists a clear one-to-one relationship between $\mathbf{u}$ and $\boldsymbol{\psi} . \quad 211$

Let $f(\mathbf{r} \mid \mathbf{u})$ be the conditional probability density function 212 (PDF) of the observation vector $\mathbf{r}=\left[r_{1}(t), r_{2}(t), \cdots, r_{N}(t)\right] 213$ conditioned on $\mathbf{u}$. Similarly, we have the conditional PDF of $\mathbf{r} 214$ conditioned on $\psi$. Then the unbiased estimate $\widehat{\mathbf{u}}$ of $\mathbf{u}$ satisfies 215 the following inequality [20]

$$
\mathrm{E}\left\{(\widehat{\mathbf{u}}-\mathbf{u})(\widehat{\mathbf{u}}-\mathbf{u})^{\mathrm{T}}\right\} \geq \mathbf{J}^{-1}(\mathbf{u}),
$$

where the Fisher information matrix (FIM) $\mathbf{J}(\mathbf{u})$ is defined by

$$
\mathbf{J}(\mathbf{u})=\mathrm{E}\left\{\frac{\partial}{\partial \mathbf{u}} \log f(\mathbf{r} \mid \mathbf{u})\left(\frac{\partial}{\partial \mathbf{u}} \log f(\mathbf{r} \mid \mathbf{u})\right)^{\mathrm{T}}\right\} .
$$

Similarly, we have the FIM of $\boldsymbol{\psi}$, denoted by $\mathbf{J}(\boldsymbol{\psi})$. The FIM 218 $\mathbf{J}(\mathbf{u})$ can be derived from $\mathbf{J}(\psi)$ according to

$$
\mathbf{J}(\mathbf{u})=\left[\begin{array}{cc}
\mathbf{D} & \mathbf{0} \\
\mathbf{0} & \mathbf{I}_{2 K M N}
\end{array}\right] \mathbf{J}(\boldsymbol{\psi})\left[\begin{array}{cc}
\mathbf{D} & \mathbf{0} \\
\mathbf{0} & \mathbf{I}_{2 K M N}
\end{array}\right]^{\mathrm{T}},
$$

where the $(2 K \times K M N)$-element block diagonal matrix $\mathbf{D} 220$ takes the following form

$$
\mathbf{D}=\operatorname{diag}\left(\mathbf{D}^{(1}, \mathbf{D}^{(2}, \cdots, \mathbf{D}^{(K)},\right.
$$

with the $(2 \times M N)$-element sub-matrix $\mathbf{D}^{(k}$ given by

$$
\begin{aligned}
& \mathbf{D}^{(k}=\left[\begin{array}{lll}
\frac{\partial \tau_{1,1}^{(k}}{\partial x_{k}} & \cdots & \frac{\partial \tau_{M, N}^{(k}}{\partial x_{k}} \\
\frac{\partial \tau_{1,1}^{(k}}{\partial y_{k}} & \cdots & \frac{\partial \tau_{M, N}^{(k}}{\partial y_{k}}
\end{array}\right] \\
& =-\frac{1}{c}\left[\begin{array}{ccc}
\cos \left(\theta_{1}^{(k}\right)+\cos \left(\varphi_{1}^{(k)}\right) & \cdots & \cos \left(\theta_{M}^{(k)}\right)+\cos \left(\varphi_{N}^{(k)}\right) \\
\sin \left(\theta_{1}^{(k}\right)+\sin \left(\varphi_{1}^{(k)}\right) & \cdots & \sin \left(\theta_{M}^{(k}\right)+\sin \left(\varphi_{N}^{(k)}\right)
\end{array}\right] .
\end{aligned}
$$

The matrix $\mathbf{C}_{x, y}$ associated with the CRLB for the unbiased 223 estimator of $\boldsymbol{l}_{x, y}$ is the $(2 K \times 2 K)$-element upper left block 224 sub-matrix of $\mathbf{J}^{-1}(\mathbf{u})$, which can be derived as [11], [21] 225

$$
\mathbf{C}_{x, y}=\left[\mathbf{J}^{-1}(\mathbf{u})\right]_{[1: 2 K ; 1: 2 K]}=\left(\mathbf{D P} \boldsymbol{\Psi} \mathbf{D}^{\mathrm{T}}\right)^{-1},
$$


where $\mathbf{P}=\mathbf{I}_{K} \otimes \operatorname{diag}(\mathbf{p}) \otimes \mathbf{I}_{N}, \quad$ and $\quad \mathbf{\Psi}=\operatorname{diag}\left(\boldsymbol{\Psi}^{(1}, \cdots\right.$, $\left.\Psi^{(K)}\right)$ is the $(K M N \times K M N)$-element block diagonal matrix with the $k$ th sub-matrix defined as

$$
\Psi^{(k}=8 \pi^{2}\left(\operatorname{diag}\left(\beta_{1}^{2}, \cdots, \beta_{M}^{2}\right) \otimes \mathbf{I}_{N}\right) \operatorname{diag}\left(\left|\mathbf{h}^{(k}\right|^{2}\right) .
$$

Let us denote the variances of the estimates of $x_{k}$ and $y_{k}$ by $\sigma_{x_{k}}^{2}$ and $\sigma_{y_{k}}^{2}$, respectively. Then we have

$$
\sum_{k=1}^{K}\left(\sigma_{x_{k}}^{2}+\sigma_{y_{k}}^{2}\right) \geq \operatorname{Tr}\left(\mathbf{C}_{x, y}\right),
$$

where $\operatorname{Tr}\left(\mathbf{C}_{x, y}\right)$ is a lower bound on the sum of the MSEs of the localization estimator $\widehat{\boldsymbol{l}}_{x, y}$. By defining $\mathbf{X}=\operatorname{diag}(\mathbf{p}) \otimes \mathbf{I}_{N}$ and noting $\mathbf{D}$ of (16), we obtain the expression of the lower bound for the $k$ th target location estimate as [12], [22]

$$
\begin{aligned}
\sum_{i=1}^{2} & {\left[\mathbf{C}_{x, y}\right]_{i+2(k-1), i+2(k-1)} } \\
& =\sum_{i=1}^{2}\left[\left(\mathbf{D P} \mathbf{\Psi} \mathbf{D}^{\mathrm{T}}\right)^{-1}\right]_{i+2(k-1), i+2(k-1)} \\
& =\operatorname{Tr}\left(\left[\begin{array}{ll}
\left(\mathbf{a}_{1,1}^{(k}\right)^{\mathrm{T}} \mathbf{p} & \left(\mathbf{a}_{1,2}^{(k}\right)^{\mathrm{T}} \mathbf{p} \\
\left(\mathbf{a}_{2,1}^{(k}\right)^{\mathrm{T}} \mathbf{p} & \left(\mathbf{a}_{2,2}^{(k}\right)^{\mathrm{T}} \mathbf{p}
\end{array}\right]^{-1}\right)=\frac{\mathbf{b}_{k}^{\mathrm{T}} \mathbf{p}}{\mathbf{p}^{\mathrm{T}} \mathbf{A}_{k} \mathbf{p}},
\end{aligned}
$$

where the second equation is obtained by first dividing the $(M N \times 2)$ matrix $\left(\mathbf{D}^{(k}\right)^{\mathrm{T}}$ into the two column vectors, $\left(\mathbf{D}^{(k}\right)^{\mathrm{T}}$ $=\left[\mathbf{d}_{1}^{(k} \mathbf{d}_{2}^{(k}\right]$, and generating the $(N \times 1)$ vectors

$$
\mathbf{d}_{i, m}^{(k}=\left[\mathbf{d}_{i}^{(k}\right]_{[(m-1) N+1: m N]}, i=1,2,1 \leq m \leq M .
$$

Then $\mathbf{a}_{i, j}^{(k}$ for $1 \leq i, j \leq 2$ are given by

$$
\begin{aligned}
& \mathbf{a}_{i, j}^{(k}=\mathrm{v}_{\operatorname{diag}}\left(\operatorname{diag}\left(\left(\mathbf{d}_{i, 1}^{(k}\right)^{\mathrm{T}}, \cdots,\left(\mathbf{d}_{i, M}^{(k)}\right)^{\mathrm{T}}\right) \boldsymbol{\Psi}^{(k}\right. \\
& \left.\times \operatorname{diag}\left(\mathbf{d}_{j, 1}^{(k}, \cdots, \mathbf{d}_{j, M}^{(k}\right)\right)
\end{aligned}
$$

while $\mathbf{b}_{k}=\mathbf{a}_{1,1}^{(k}+\mathbf{a}_{2,2}^{(k}$ and $\mathbf{A}_{k}=\mathbf{a}_{1,1}^{(k}\left(\mathbf{a}_{2,2}^{(k)}\right)^{\mathrm{T}}-\mathbf{a}_{1,2}^{(k}\left(\mathbf{a}_{2,1}^{(k}\right)^{\mathrm{T}}$

Our task is to design a beneficial power allocation strategy capable of achieving a localization accuracy threshold $\eta$. We can use the weighting $v_{k}$ to indicate the localization accuracy requirement for the $k$ th target. The larger $v_{k}$ is, the higher accuracy is required for the $k$ th target. For a predetermined lower bound of total MSE of all the targets, the transmit power of the different transmit radars can then be appropriately allocated for minimizing the total transmit power. This can be formulated as the following optimization problem $\mathbb{P} 1$

$$
\begin{aligned}
& \min _{\mathbf{p}} \mathbf{1}^{\mathrm{T}} \mathbf{p} \\
& \mathbb{P} 1 \text { : } \quad \text { s.t. } \sum_{k=1}^{K} v_{k} \frac{\mathbf{b}_{k}^{\mathrm{T}} \mathbf{p}}{\mathbf{p}^{\mathrm{T}} \mathbf{A}_{k} \mathbf{p}} \leq \eta, \\
& p_{m_{\text {min }}} \leq p_{m} \leq p_{m_{\text {max }}}, 1 \leq m \leq M \text {. }
\end{aligned}
$$

Because generally speaking $\mathbf{A}_{k}$ is not a positive definite matrix, the optimization $\mathbb{P} 1$ is a nonconvex problem.
In [13], a similar optimization problem is formulated as

$$
\begin{array}{ll}
\min _{\mathbf{p}} & \mathbf{1}^{\mathrm{T}} \mathbf{p}, \\
\text { s.t. } & \frac{\mathbf{b}_{k}^{\mathrm{T}} \mathbf{p}}{\mathbf{p}^{\mathrm{T}} \mathbf{A}_{k} \mathbf{p}} \leq \bar{\eta}, 1 \leq k \leq K, \\
& p_{m_{\min }} \leq p_{m} \leq p_{m_{\max }}, 1 \leq m \leq M,
\end{array}
$$

given an equivalent localization accuracy threshold $\bar{\eta}$. In [13], 252 a Taylor series based technique is applied to approximate the 253 inequality constraints in (25) in order to relax the nonconvex 254 optimization problem for the sake of obtaining a solution. Intu- 255 itively, the cost function associated with an optimal solution of 256 our problem $\mathbb{P} 1$ of (24) is generally smaller than that associated 257 with an optimal solution of (25), i.e., we can achieve a lower 258 power consumption. This is achieved at the potential cost of a 259 slightly reduced localization accuracy.

\section{POWER Resource Allocation}

\section{A. Multi-Target Case}

In order to solve the nonconvex problem $\mathbb{P} 1$ of (24), we have 263 to change it into a simpler form. Specifically, we have to change the inequality constraint into an equality one, i.e.,

$$
\sum_{k=1}^{K} v_{k} \frac{\mathbf{b}_{k}^{\mathrm{T}} \mathbf{p}}{\mathbf{p}^{\mathrm{T}} \mathbf{A}_{k} \mathbf{p}} \leq \eta \Rightarrow \sum_{k=1}^{K} v_{k} \frac{\mathbf{b}_{k}^{\mathrm{T}} \mathbf{p}}{\mathbf{p}^{\mathrm{T}} \mathbf{A}_{k} \mathbf{p}}=\eta .
$$

Lemma 1: An increase of the transmit power $\mathbf{p}$ results in a 266 reduction of the MSE, namely,

$$
\sum_{k=1}^{K} v_{k} \frac{\mathbf{b}_{k}^{\mathrm{T}}(\mathbf{p}+\Delta \mathbf{p})}{(\mathbf{p}+\Delta \mathbf{p})^{\mathrm{T}} \mathbf{A}_{k}(\mathbf{p}+\Delta \mathbf{p})} \leq \sum_{k=1}^{K} v_{k} \frac{\mathbf{b}_{k}^{\mathrm{T}} \mathbf{p}}{\mathbf{p}^{\mathrm{T}} \mathbf{A}_{k} \mathbf{p}} .
$$

The proof of Lemma 1 is similar to that of single-target case 268 given in [12]. Thus, to achieve a reduced power consumption, 269 we can always set the MSE to its maximum tolerance. The 270 change of constraint as given in (26) leads to the problem $\mathbb{P} 2$,

$$
\begin{aligned}
& \min _{\mathbf{p}} \\
\mathbb{P} 2: & \mathbf{1}^{\mathrm{T}} \mathbf{p}, \\
\text { s.t. } & \sum_{k=1}^{K} v_{k} \frac{\mathbf{b}_{k}^{\mathrm{T}} \mathbf{p}}{\mathbf{p}^{\mathrm{T}} \mathbf{A}_{k} \mathbf{p}}=\eta, \\
& p_{m_{\text {min }}} \leq p_{m} \leq p_{m_{\text {max }}}, 1 \leq m \leq M .
\end{aligned}
$$

Theorem 1: The solutions of $\mathbb{P} 1$ and $\mathbb{P} 2$ are identical.

The proof of Theorem 1 is straightforward. By introducing the auxiliary variables

$$
w_{k}=\frac{1}{\eta \mathbf{p}^{\mathrm{T}} \mathbf{A}_{k} \mathbf{p}}, 1 \leq k \leq K,
$$

and their corresponding lower and upper bounds

$$
w_{k_{\min }}=\frac{1}{\eta \mathbf{p}_{\max }^{\mathrm{T}} \mathbf{A}_{k} \mathbf{p}_{\max }}, w_{k_{\max }}=\frac{1}{\eta \mathbf{p}_{\min }^{\mathrm{T}} \mathbf{A}_{k} \mathbf{p}_{\min }}, 1 \leq k \leq K,
$$


$\mathbb{P} 2$ is reformulated as the following optimization problem $\mathbb{P} 3$ :

$$
\begin{aligned}
\min _{\mathbf{p}, \mathbf{w}} & \mathbf{1}^{\mathrm{T}} \mathbf{p}, \\
\mathbb{P} 3: \quad \text { s.t. } & \sum_{k=1}^{K} v_{k} w_{k} \mathbf{b}_{k}^{\mathrm{T}} \mathbf{p}=1, \\
& w_{k} \eta \mathbf{p}^{\mathrm{T}} \mathbf{A}_{k} \mathbf{p}=1,1 \leq k \leq K, \\
& p_{m_{\min }} \leq p_{m} \leq p_{m_{\max }}, 1 \leq m \leq M, \\
& w_{k_{\min }} \leq w_{k} \leq w_{k_{\max }}, 1 \leq k \leq K .
\end{aligned}
$$

The following corollary is obvious.

Corollary 1: If $\mathbf{p}^{\star}$ associated with $w_{k}^{\star}=\frac{1}{\eta\left(\mathbf{p}^{\star}\right)^{T} \mathbf{A}_{k} \mathbf{p}^{\star}}$ for $1 \leq k \leq K$ is an optimal solution of the problem $\mathbb{P} 3(31)$, $\mathbf{p}^{\star}$ is an optimal solution for the problem $\mathbb{P} 1$ of (24). Conversely, if $\mathbf{p}^{\star}$ is an optimal solution of the problem $\mathbb{P} 1$, together with $w_{k}^{\star}=\frac{1}{\eta\left(\mathbf{p}^{\star}\right)^{\mathrm{T}} \mathbf{A}_{k} \mathbf{p}^{\star}}$ for $1 \leq k \leq K$ it is an optimal solution of the problem $\mathbb{P} 3$.

1) OCD-based method: The Lagrangian associated with the optimization problem $\mathbb{P} 3$ is

$$
\begin{aligned}
L(\mathbf{p}, \mathbf{w}, \lambda, \boldsymbol{\mu})= & \mathbf{1}^{\mathrm{T}} \mathbf{p}+\lambda\left(\sum_{k=1}^{K} v_{k} w_{k} \mathbf{b}_{k}^{\mathrm{T}} \mathbf{p}-1\right) \\
& +\sum_{k=1}^{K} \mu_{k}\left(w_{k} \eta \mathbf{p}^{\mathrm{T}} \mathbf{A}_{k} \mathbf{p}-1\right),
\end{aligned}
$$

with $\mathbf{w}=\left[w_{1} w_{2} \cdots w_{K}\right]^{\mathrm{T}}$ and $\boldsymbol{\mu}=\left[\mu_{1} \mu_{2} \cdots \mu_{K}\right]^{\mathrm{T}}$, where $\lambda$ and $\mu_{k}$ for $1 \leq k \leq K$ are Lagrangian multipliers. We optimize the Lagrangian (32) with respect to $\mathbf{p}, \lambda, w_{k}$ and $\mu_{k}$. Using the steepest descent method, the search directions are related to the Karush-Kuhn-Tucker (KKT) conditions by

$$
\begin{aligned}
\Delta \mathbf{p}= & \nabla_{\mathbf{p}} L(\mathbf{p}, \mathbf{w}, \lambda, \boldsymbol{\mu})=\mathbf{1}+\lambda\left(\sum_{k=1}^{K} w_{k} v_{k} \mathbf{b}_{k}\right) \\
& +\sum_{k=1}^{K} \mu_{k} w_{k} \eta\left(\mathbf{A}_{k}+\mathbf{A}_{k}^{\mathrm{T}}\right) \mathbf{p}, \\
\Delta \lambda= & \nabla_{-\lambda} L(\mathbf{p}, \mathbf{w}, \lambda, \boldsymbol{\mu})=-\sum_{k=1}^{K} w_{k} v_{k} \mathbf{b}_{k}^{\mathrm{T}} \mathbf{p}+1, \\
\Delta w_{k}= & \nabla_{w_{k}} L(\mathbf{p}, \mathbf{w}, \lambda, \boldsymbol{\mu}) \\
= & \lambda v_{k} \mathbf{b}_{k}^{\mathrm{T}} \mathbf{p}+\mu_{k} \eta \mathbf{p}^{\mathrm{T}} \mathbf{A}_{k} \mathbf{p}, 1 \leq k \leq K, \\
\Delta \mu_{k}= & \nabla_{-\mu_{k}} L(\mathbf{p}, \mathbf{w}, \lambda, \boldsymbol{\mu}) \\
= & -\left(\eta w_{k} \mathbf{p}^{\mathrm{T}} \mathbf{A}_{k} \mathbf{p}+1\right), 1 \leq k \leq K,
\end{aligned}
$$

291 where we have $\Delta \mathbf{p}=\left[\Delta p_{1} \Delta p_{2} \cdots \Delta p_{M}\right]^{\mathrm{T}}$. The primal and

$$
\begin{aligned}
& p_{m}^{(n+1)}=\left[p_{m}^{(n)}-\kappa_{1} \Delta p_{m}^{(n)}\right]_{p_{m_{\min }}}^{p_{m_{\max }}}, 1 \leq m \leq M, \\
& \lambda^{(n+1)}=\lambda^{(n)}-\kappa_{2} \Delta \lambda^{(n)}, \\
& w_{k}^{(n+1)}=w_{k}^{(n)}-\kappa_{3} \Delta w_{k}^{(n)}, 1 \leq k \leq K, \\
& \mu_{k}^{(n+1)}=\mu_{k}^{(n)}-\kappa_{4} \Delta \mu_{k}^{(n)}, 1 \leq k \leq K,
\end{aligned}
$$

where the superscript ${ }^{(n)}$ denotes the iteration index and

$$
[a]_{b}^{c}=\min \{\max \{a, b\}, c\},
$$

while $\kappa_{i}$ for $1 \leq i \leq 4$ represent the step sizes for the primal 294 variables $\mathbf{p}$, the dual variable $\lambda$, the primal variables $\mathbf{w}$ and the 295 dual variables $\boldsymbol{\mu}$, respectively. According to [23], an exponen- 296 tially decreasing step size is highly desired. Furthermore, since 297 $\mathrm{p}, \lambda, \mathbf{w}$ and $\boldsymbol{\mu}$ have very different properties and their impacts 298 on the Lagrangian are 'unequal', using different step sizes for 299 them makes sense. By combining these two considerations, we 300 set the four step sizes for $\mathbf{p}, \lambda$, $\mathbf{w}$ and $\boldsymbol{\mu}$ according to

$$
\kappa_{i}=c_{i} e^{-\alpha n} \text { with } 0 \leq \alpha \ll 1 \text {, for } 1 \leq i \leq 4,
$$

where $c_{i}>0$ for $1 \leq i \leq 4$ are different constants.

The choice of the initial values for the primal variables $p_{m}, \quad 303$ $1 \leq m \leq M$, influences the convergence performance. Ideally, 304 they should be chosen to be close to their own specific optimal 305 values so as to enhance the convergence speed. For practical 306 reason, the initialization should be easy and simple to realize 307 too. Hence we opt for the initial powers of

$$
\mathbf{p}^{(0)}=\mathbf{p}_{\text {equ }}=\frac{1}{\eta} \sum_{k=1}^{K} v_{k} \frac{\mathbf{b}_{k}^{\mathrm{T}} \mathbf{1}}{\mathbf{1}^{\mathrm{T}} \mathbf{A}_{k} \mathbf{1}} \mathbf{1},
$$

which is obtained by setting all the elements of $\mathbf{p}$ to be equal. 309 Then, $w_{k}$ is initialized according to

$$
w_{k}^{(0)}=\frac{1}{\eta \mathbf{p}_{\text {equ }}^{\mathrm{T}} \mathbf{A}_{k} \mathbf{p}_{\text {equ }}}, 1 \leq k \leq K .
$$

The iterative procedure of (37) to (40) is repeated until 311 $\left\|\mathbf{p}^{(n+1)}-\mathbf{p}^{(n)}\right\|$ becomes smaller than a preset small positive number or the maximum number of iterations is reached.

Remark 1: It is difficult to find a closed-form solution from the set of KKT conditions, because $\mathbf{A}_{k}$ for $1 \leq k \leq K$ are generally non-invertible. Hence our algorithm finds a locally optimal point in an iterative manner. It is also worth noting that the standard OCD [17] is typically based on a Newtontype algorithm, but our proposed OCD method is a steepest descent algorithm. The reason is that the Hessian matrix for the Lagrangian $L(\mathbf{p}, \mathbf{w}, \lambda, \boldsymbol{\mu})$ of (32) is not invertible.

2) ADMM-based method: ADMM was originally proposed for solving convex problems in a parallel manner [18]. Let us now discuss how to apply the ADMM method for solving the nonconvex problem $\mathbb{P} 3$. By introducing an auxiliary vector $\mathbf{z}=$ p, (29) can be rewritten as

$$
\mathbf{p}=\mathbf{z} \text { and } \eta w_{k} \mathbf{z}^{\mathrm{T}} \mathbf{A}_{k} \mathbf{p}=1,1 \leq k \leq K .
$$

Therefore, $\mathbb{P} 3$ can be reformulated into the problem $\mathbb{P} 4$ :

$\mathbb{P} 4$ :

$$
\begin{aligned}
\min _{\mathbf{p}, \mathbf{w}, \mathbf{z}} & \mathbf{1}^{\mathrm{T}} \mathbf{p}, \\
\text { s.t. } & \sum_{k=1}^{K} v_{k} w_{k} \mathbf{b}_{k}^{\mathrm{T}} \mathbf{p}=1, \\
& \mathbf{p}=\mathbf{z}, \\
& w_{k} \eta \mathbf{z}^{\mathrm{T}} \mathbf{A}_{k} \mathbf{p}=1,1 \leq k \leq K, \\
& p_{m_{\min }} \leq p_{m} \leq p_{m_{\max }}, 1 \leq m \leq M, \\
& w_{k_{\min }} \leq w_{k} \leq w_{k_{\max }}, 1 \leq k \leq K .
\end{aligned}
$$


This problem is convex with respect to $\mathbf{p}, \mathbf{z}$ and $w_{k}$, respectively. An augmented Lagrangian is constructed as follows

$$
\begin{aligned}
& L\left(\mathbf{p}, \mathbf{w}, \mathbf{z}, \mathbf{d}_{0}, d_{1}, \mathbf{d}_{2}\right)=\mathbf{1}^{\mathrm{T}} \mathbf{p}+\frac{\rho_{0}}{2}\|\mathbf{p}-\mathbf{z}\|^{2}+\mathbf{d}_{0}^{\mathrm{T}}(\mathbf{p}-\mathbf{z}) \\
& +\sum_{k=1}^{K} \frac{\rho_{2, k}}{2}\left|w_{k} \mathbf{z}^{\mathrm{T}} \mathbf{A}_{k} \mathbf{p} \eta-1\right|^{2}+\sum_{k=1}^{K} d_{2, k}\left(w_{k} \mathbf{z}^{\mathrm{T}} \mathbf{A}_{k} \mathbf{p} \eta-1\right) \\
& +\frac{\rho_{1}}{2}\left|\sum_{k=1}^{K} w_{k} v_{k} \mathbf{b}_{k}^{\mathrm{T}} \mathbf{p}-1\right|^{2}+d_{1}\left(\sum_{k=1}^{K} w_{k} v_{k} \mathbf{b}_{k}^{\mathrm{T}} \mathbf{p}-1\right)
\end{aligned}
$$

where $\mathbf{d}_{0}=\left[d_{0,1} \cdots d_{0, M}\right]^{\mathrm{T}}, d_{1}$ and $\mathbf{d}_{2}=\left[d_{2,1} \cdots d_{2, K}\right]^{\mathrm{T}}$ are the dual variables corresponding to the constraints $\mathbf{p}=\mathbf{z}$, $\sum_{k=1}^{K} w_{k} v_{k} \mathbf{b}_{k}^{\mathrm{T}} \mathbf{p}=1$ and $w_{k} \mathbf{z}^{\mathrm{T}} \mathbf{A}_{k} \mathbf{p} \eta=1$ for $1 \leq k \leq K$, respectively, while $\rho_{0}, \rho_{1}$ and $\boldsymbol{\rho}_{2}=\left[\rho_{2,1} \cdots \rho_{2, K}\right]^{\mathrm{T}}$ are the penalty parameters. Note that the augmented Lagrangian (47) is quadratic. For convenience, we scale the dual variables as $\mathbf{e}=\frac{1}{\rho_{0}} \mathbf{d}_{0}, \mu=\frac{1}{\rho_{1}} d_{1}$ and $\gamma=\left[\gamma_{1} \cdots \gamma_{K}\right]^{\mathrm{T}}$ with $\gamma_{k}=\frac{1}{\rho_{2, k}} d_{2, k}$ for $1 \leq k \leq K$. Then, from (47) we obtain the following augmented Lagrangian

$$
\begin{gathered}
L(\mathbf{p}, \mathbf{w}, \mathbf{z}, \mathbf{e}, \mu, \gamma)=\mathbf{1}^{\mathrm{T}} \mathbf{p}+\frac{\rho_{0}}{2}\|\mathbf{p}-\mathbf{z}+\mathbf{e}\|^{2}-\frac{\rho_{0}}{2}\|\mathbf{e}\|^{2} \\
+\sum_{k=1}^{K} \frac{\rho_{2, k}}{2}\left|w_{k} \mathbf{z}^{\mathrm{T}} \mathbf{A}_{k} \mathbf{p} \eta-1+\gamma_{k}\right|^{2}-\sum_{k=1}^{K} \frac{\rho_{2, k}}{2}\left|\gamma_{k}\right|^{2} \\
+\frac{\rho_{1}}{2}\left|\sum_{k=1}^{K} w_{k} v_{k} \mathbf{b}_{k}^{\mathrm{T}} \mathbf{p}-1+\mu\right|^{2}-\frac{\rho_{1}}{2}|\mu|^{2}
\end{gathered}
$$

We can find the saddle point of the augmented Lagrangian (48) by minimizing the Lagrangian over the primal variables $\mathbf{p}, \mathbf{w}$ and $\mathbf{z}$, as well as maximizing it over the dual variables $\mathbf{e}, \mu$ and $\gamma$, in an alternative way. In particular, we update the primal variables $\mathbf{p}, \mathbf{w}$ and $\mathbf{z}$ separately one by one. Furthermore, after the update of the dual variables e, $\mu$ and $\gamma$, we adjust the penalty parameters $\rho_{0}, \rho_{1}$ and $\rho_{2}$. We now summarize our ADMMbased procedure.

Initialization: Let us also opt for the equal power initialization $\mathbf{p}^{(0)}=\mathbf{p}_{e q u}$ of (43). The other primal variables are initialized as $w_{k}^{(0)}=\frac{1}{\eta \mathbf{p}_{e q u}^{\mathrm{T}} \mathbf{A}_{k} \mathbf{p}_{e q u}}$ for $1 \leq k \leq K$ of (44), and

$$
\mathbf{z}^{(0)}=\mathbf{p}_{e q u} .
$$

The initial penalty parameters, $\rho_{0}^{(0)}, \rho_{1}^{(0)}$ and $\rho_{2, k}^{(0)}$ for $1 \leq k \leq$ $K$, are typically set to a large positive value, say, 500 . Next, the dual variables are initialized as follows. Choose $\mu^{(0)}=1$ and $\gamma_{k}^{(0)}=1$ for $1 \leq k \leq K$, while every element of $\mathbf{e}^{(0)}$ is set to 1 too. Then we set the iteration index $n=0$.

Iterative Procedure: At the $(n+1)$ th iteration, perform:
- Step 1: Update the primal variables p. Upon isolating all the terms involving $\mathbf{p}$ in the Lagrangian (48), we have

$$
\begin{aligned}
\min _{\mathbf{p}} & \mathbf{1}^{\mathrm{T}} \mathbf{p}+\frac{\rho_{0}^{(n)}}{2}\left\|\mathbf{p}-\mathbf{z}^{(n)}+\mathbf{e}^{(n)}\right\|^{2} \\
& +\frac{\rho_{1}^{(n)}}{2}\left|\sum_{k=1}^{K} w_{k}^{(n)} v_{k} \mathbf{b}_{k}^{\mathrm{T}} \mathbf{p}-1+\mu^{(n)}\right|^{2} \\
& +\sum_{k=1}^{K} \frac{\rho_{2, k}^{(n)}}{2}\left|w_{k}^{(n)}\left(\mathbf{z}^{(n)}\right)^{\mathrm{T}} \mathbf{A}_{k} \mathbf{p} \eta-1+\gamma_{k}^{(n)}\right|^{2}, \\
\text { s.t. } & p_{m_{\min }} \leq p_{m} \leq p_{m_{\max }}, 1 \leq m \leq M,
\end{aligned}
$$

which is a constrained convex optimization. Setting the 358 derivative of the objective function to zero yields the $(n+359$ 1)th estimate of $\mathbf{p}$ as follows. First compute

$$
\begin{aligned}
\overline{\mathbf{p}}^{(n+1)=} & {\left[\bar{p}_{1}^{(n+1)} \cdots \bar{p}_{M}^{(n+1)}\right]^{\mathrm{T}}=\left(\mathbf{P}_{1}^{(n+1)}\right)^{-1} \mathbf{p}_{2}^{(n+1)}, } \\
\mathbf{P}_{1}^{(n+1)}= & \rho_{0}^{(n)} \mathbf{I}_{M}+\rho_{1}^{(n)}\left(\sum_{k=1}^{K} w_{k}^{(n)} v_{k} \mathbf{b}_{k}\right) \\
& \times\left(\sum_{k=1}^{K} w_{k}^{(n)} v_{k} \mathbf{b}_{k}^{\mathrm{T}}\right)+\sum_{k=1}^{K} \rho_{2, k}^{(n)} \\
& \times\left(w_{k}^{(n)}\left(\mathbf{A}_{k}\right)^{\mathrm{T}} \mathbf{z}^{(n)} \eta\right)\left(w_{k}^{(n)}\left(\mathbf{z}^{(n)}\right)^{\mathrm{T}} \mathbf{A}_{k} \eta\right)^{\mathrm{T}}, \\
\mathbf{p}_{2}^{(n+1)}= & -\mathbf{1}+\rho_{0}^{(n)}\left(\mathbf{z}^{(n)}+\mathbf{e}^{(n)}\right) \\
& +\rho_{1}^{(n)}\left(\sum_{k=1}^{K} w_{k}^{(n)} v_{k} \mathbf{b}_{k}\right)\left(1-\mu^{(n)}\right) \\
& +\rho_{2, k}^{(n)}\left(w_{k}^{(n)}\left(\mathbf{A}_{k}\right)^{\mathrm{T}} \mathbf{z}^{(n)} \eta\right)\left(1-\gamma_{k}^{(n)}\right) . \quad(53)
\end{aligned}
$$

The final estimate is then given by

$$
p_{m}^{(n+1)}=\left[\bar{p}_{m}^{(n+1)}\right]_{p_{m \text { min }}}^{p_{m \text { max }}}, 1 \leq m \leq M .
$$

- Step 2: Update the primal variables $\mathrm{w}$. The optimization involving $\mathbf{w}$ is also a constrained convex problem

$$
\begin{aligned}
\min _{\mathbf{w}} & \frac{\rho_{1}^{(n)}}{2}\left|\sum_{k=1}^{K} w_{k} v_{k} \mathbf{b}_{k}^{\mathrm{T}} \mathbf{p}^{(n+1)}-1+\mu^{(n)}\right|^{2} \\
& +\sum_{k=1}^{K} \frac{\rho_{2, k}^{(n)}}{2}\left|w_{k}\left(\mathbf{z}^{(n)}\right)^{\mathrm{T}} \mathbf{A}_{k} \mathbf{p}^{(n+1)} \eta-1+\gamma_{k}^{(n)}\right|^{2}, \\
\text { s.t. } & w_{k_{\min }} \leq w_{k} \leq w_{k_{\max }}, 1 \leq k \leq K .
\end{aligned}
$$

The solution is given by

$$
w_{k}^{(n+1)}=\left[\frac{w_{k, 1}^{(n+1)}}{w_{k, 2}^{(n+1)}}\right]_{w_{k_{\min }}}^{w_{k_{\max }}}, 1 \leq k \leq K,
$$


where

$$
\begin{aligned}
w_{k, 1}^{(n+1)}= & \rho_{1}^{(n)} v_{k} \mathbf{b}_{k}^{\mathrm{T}} \mathbf{p}^{(n+1)}\left(1-\mu^{(n)}-\sum_{k^{\prime} \neq k} v_{k^{\prime}} \mathbf{b}_{k^{\prime}}^{\mathrm{T}} \mathbf{p}^{(n+1)}\right) \\
& +\rho_{2, k}^{(n)}\left(\left(\mathbf{z}^{(n)}\right)^{\mathrm{T}} \mathbf{A}_{k} \mathbf{p}^{(n+1)} \eta\right)\left(1-\gamma_{k}^{(n)}\right) \\
w_{k, 2}^{(n+1)}= & \rho_{1}^{(n)}\left(v_{k} \mathbf{b}_{k}^{\mathrm{T}} \mathbf{p}^{(n+1)}\right)^{2} \\
& +\rho_{2, k}^{(n)}\left(\left(\mathbf{z}^{(n)}\right)^{\mathrm{T}} \mathbf{A}_{k} \mathbf{p}^{(n+1)} \eta\right)^{2} .
\end{aligned}
$$

- Step 3: Update the primal variables $\mathbf{z}$. Isolating all the terms involving $\mathbf{z}$, the optimization is an unconstrained convex problem

$$
\begin{aligned}
\min _{\mathbf{z}} & \frac{\rho_{0}^{(n)}}{2}\left\|\mathbf{p}^{(n+1)}-\mathbf{z}+\mathbf{e}^{(n)}\right\|^{2} \\
& +\sum_{k=1}^{K} \frac{\rho_{2, k}^{(n)}}{2}\left|w_{k}^{(n+1)} \mathbf{z}^{\mathrm{T}} \mathbf{A}_{k} \mathbf{p}^{(n+1)} \eta-1+\gamma_{k}^{(n)}\right|^{2} .
\end{aligned}
$$

Solving (59) yields the $(n+1)$ th estimate of $\mathbf{z}$ as

$$
\mathbf{z}^{(n+1)}=\left(\mathbf{Z}_{1}^{(n+1)}\right)^{-1} \mathbf{z}_{2}^{(n+1)}
$$

where

$$
\begin{aligned}
\mathbf{Z}_{1}^{(n+1)}= & \rho_{0}^{(n)} \mathbf{I}_{M}+\sum_{k=1}^{K} \rho_{2, k}^{(n)}\left(w_{k}^{(n+1)} \mathbf{A}_{k} \mathbf{p}^{(n+1)} \eta\right) \\
& \times\left(w_{k}^{(n+1)} \mathbf{A}_{k} \mathbf{p}^{(n+1)} \eta\right)^{\mathrm{T}}, \\
\mathbf{z}_{2}^{(n+1)}= & \rho_{0}^{(n)}\left(\mathbf{p}^{(n+1)}+\mathbf{e}^{(n)}\right) \\
& +\sum_{k=1}^{K} \rho_{2, k}^{(n)}\left(w_{k}^{(n+1)} \mathbf{A}_{k} \mathbf{p}^{(n+1)} \eta\right)\left(1-\gamma_{k}^{(n)}\right) .
\end{aligned}
$$

- Step 4: Update the dual variables e, $\mu$ and $\gamma$. Maximizing the Lagrangian (48) with respect to the dual variables yields

$$
\begin{aligned}
\mathbf{e}^{(n+1)}= & \mathbf{e}^{(n)}+\mathbf{p}^{(n+1)}-\mathbf{z}^{(n+1)} \\
\mu^{(n+1)}= & \mu^{(n)}+\sum_{k=1}^{K} w_{k}^{(n+1)} v_{k} \mathbf{b}_{k}^{\mathrm{T}} \mathbf{p}^{(n+1)}-1 \\
\gamma_{k}^{(n+1)}= & \gamma_{k}^{(n)}+w_{k}^{(n+1)}\left(\mathbf{z}^{(n+1)}\right)^{\mathrm{T}} \mathbf{A}_{k} \mathbf{p}^{(n+1)} \eta-1 \\
& 1 \leq k \leq K
\end{aligned}
$$

- Step 5: Update the penalty parameters $\rho_{0}, \rho_{1}$ and $\boldsymbol{\rho}_{2}$. The penalty parameters are updated at the end of each iteration for the first a few iterations to speed up the convergence. At the $(n+1)$ th iteration, associated with the three penalty parameters of $\rho_{0}^{(n)}, \rho_{1}^{(n)}$ and $\rho_{2}^{(n)}$, we have three primal residuals

$$
\begin{aligned}
r_{0}^{(n+1)}= & \left\|\mathbf{p}^{(n+1)}-\mathbf{z}^{(n+1)}\right\|, \\
r_{1}^{(n+1)}= & \left|\sum_{k=1}^{K} w_{k}^{(n+1)} v_{k} \mathbf{b}_{k}^{\mathrm{T}} \mathbf{p}^{(n+1)}-1\right|, \\
r_{2, k}^{(n+1)}= & \left|w_{k}\left(\mathbf{z}^{(n+1)}\right)^{\mathrm{T}} \mathbf{A}_{k} \mathbf{p}^{(n+1)} \eta-1\right|, \\
& 1 \leq k \leq K,
\end{aligned}
$$

as well as three respective dual residuals

$$
\begin{aligned}
& s_{0}^{(n+1)}=\left\|\rho_{0}^{(n)}\left(\mathbf{z}^{(n+1)}-\mathbf{z}^{(n)}\right)\right\|, \\
& s_{1}^{(n+1)}=\left\|\mathbf{s}_{1 a}^{(n+1)}\right\|, \\
& s_{2, k}^{(n+1)}=\sqrt{\left(s_{2 a, k}^{(n+1)}\right)^{2}+\left\|\mathbf{s}_{2 b, k}^{(n+1)}\right\|}, 1 \leq k \leq K,
\end{aligned}
$$

where

$$
\begin{aligned}
\mathbf{s}_{1 a}^{(n+1)}= & \mu^{(n+1)} \rho_{1}^{(n)}\left(\sum_{k=1}^{K}\left(w_{k}^{(n)}-w_{k}^{(n+1)}\right) v_{k} \mathbf{b}_{k}\right) \\
& +\rho_{1}^{(n)}\left(\sum_{k=1}^{K} w_{k}^{(n)} v_{k} \mathbf{b}_{k}\right) \\
& \times\left(\sum_{k=1}^{K}\left(w_{k}^{(n)}-w_{k}^{(n+1)}\right) v_{k} \mathbf{b}_{k}^{\mathrm{T}} \mathbf{p}^{(n+1)}\right),
\end{aligned}
$$

$$
\begin{aligned}
s_{2 a, k}^{(n+1)}= & \rho_{2, k}^{(n)}\left(\mathbf{z}^{(n)}\right)^{\mathrm{T}} \mathbf{A}_{k} \mathbf{p}^{(n+1)} \eta \\
& \times\left(w_{k}^{(n+1)}\left(\mathbf{z}^{(n)}-\mathbf{z}^{(n+1)}\right)^{\mathrm{T}} \mathbf{A}_{k} \mathbf{p}^{(n+1)} \eta-1\right) \\
& +\gamma_{k}^{(n+1)} \rho_{2, k}^{(n)}\left(\left(\mathbf{z}^{(n)}-\mathbf{z}^{(n+1)}\right)^{\mathrm{T}} \mathbf{A}_{k} \mathbf{p}^{(n+1)} \eta\right), \\
\mathbf{s}_{2 b, k}^{(n+1)}= & \rho_{2, k}^{(n)} w_{k}^{(n)} \eta \mathbf{A}_{k}^{\mathrm{T}} \mathbf{z}^{(n)} \\
& \times\left(\left(w_{k}^{(n)}\left(\mathbf{z}^{(n)}\right)^{\mathrm{T}}-w_{k}^{(n+1)}\left(\mathbf{z}^{(n+1)}\right)^{\mathrm{T}}\right)\right. \\
& \left.\times \mathbf{A}_{k} \mathbf{p}^{(n+1)} \eta\right)+\gamma_{k}^{(n+1)} \rho_{2, k}^{(n)} \eta \mathbf{A}_{k}^{\mathrm{T}} \\
& \times\left(w_{k}^{(n)} \mathbf{z}^{(n)}-w_{k}^{(n+1)} \mathbf{z}^{(n+1)}\right) .
\end{aligned}
$$

The exact definitions of the dual residuals can be found in 381 Appendix A.

The penalty parameter $\rho_{0}$ is updated as follows

$$
\rho_{0}^{(n+1)}= \begin{cases}\tau \rho_{0}^{(n)}, & \text { if } r_{0}^{(n+1)} \geq \varepsilon s_{0}^{(n+1)}, \\ \frac{1}{\tau} \rho_{0}^{(n)}, & \text { if } s_{0}^{(n+1)} \geq \varepsilon r_{0}^{(n+1)}, \\ \rho_{0}^{(n)}, & \text { otherwise, }\end{cases}
$$

where the scalars $\tau>1$ and $\varepsilon>1$ with typical values of 384 $\tau=2$ and $\varepsilon=10$. The idea behind this penalty parameter 385 update is to balance the primal and dual residual norms 386 $r_{0}^{(n+1)}$ and $s_{0}^{(n+1)}$, i.e., to keep $\frac{r_{0}^{(n+1)}}{s_{0}^{(n+1)}} \leq \varepsilon$ and $\frac{s_{0}^{(n+1)}}{r_{0}^{(n+1)}} \leq \varepsilon, \quad 387$ 
as they both converge to zero [18], [25]. The related dual variables are rescaled to remove the impact of changing $\rho_{0}$ according to

$$
\mathbf{e}^{(n+1)}=\frac{\rho_{0}^{(n)}}{\rho_{0}^{(n+1)}} \mathbf{e}^{(n)} .
$$

Similarly, $\rho_{1}$ is updated according to

$$
\rho_{1}^{(n+1)}=\left\{\begin{array}{cl}
\tau \rho_{1}^{(n)}, & \text { if } r_{1}^{(n+1)} \geq \varepsilon s_{1}^{(n+1)}, \\
\frac{1}{\tau} \rho_{1}^{(n)}, & \text { if } s_{1}^{(n+1)} \geq \varepsilon r_{1}^{(n+1)}, \\
\rho_{1}^{(n)}, & \text { otherwise. }
\end{array}\right.
$$

The related dual variable is then scaled according to

$$
\mu^{(n+1)}=\frac{\rho_{1}^{(n)}}{\rho_{1}^{(n+1)}} \mu^{(n)} .
$$

Likewise, $\rho_{2, k}$ for $1 \leq k \leq K$ are updated according to

$$
\rho_{2, k}^{(n+1)}= \begin{cases}\tau \rho_{2, k}^{(n)}, & \text { if } r_{2, k}^{(n+1)} \geq \varepsilon s_{2, k}^{(n+1)}, \\ \frac{1}{\tau} \rho_{2, k}^{(n)}, & \text { if } s_{2, k}^{(n+1)} \geq \varepsilon r_{2, k}^{(n+1)}, \\ \rho_{2, k}^{(n)}, & \text { otherwise, }\end{cases}
$$

and the corresponding dual variables are rescaled as

$$
\gamma_{k}^{(n+1)}=\frac{\rho_{2, k}^{(n)}}{\rho_{2, k}^{(n+1)}} \gamma_{k}^{(n)}, 1 \leq k \leq K .
$$

- Termination of the iterative procedure. The iterative procedure is terminated when $\left\|\mathbf{p}^{(n+1)}-\mathbf{p}^{(n)}\right\|$ becomes smaller than a predefined small positive value or the preset maximum number of iterations is reached. Otherwise, set $n=n+1$ and go to Step 1 .

Remark 2: The ADMM combines the advantages of the dual ascent and the augmented Lagrangian method. The dual ascent approach deals with the complicated constraints, while the augmented Lagrangian method is capable of enhancing the convergence rate and the robustness of the algorithm.

Remark 3: We deal with the optimization problem (24), and in every iteration of our OCD and ADMM methods, we have a closed-form update value. By contrast, Garcia et al. [13] deal with the optimization problem (25), and in every iteration, an inner iterative loop is required for computing an updated value by the algorithm of [13].

\section{B. Single-Target Case}

The target index $k$ can be dropped and then the optimization is simplified to the problem $\mathbb{P} 5$

$$
\begin{array}{cc}
\min _{\mathbf{p}} & \mathbf{1}^{\mathrm{T}} \mathbf{p}, \\
\mathbb{P} 5: \quad \text { s.t. } & \frac{\mathbf{b}^{\mathrm{T}} \mathbf{p}}{\mathbf{p}^{\mathrm{T}} \mathbf{A} \mathbf{p}} \leq \eta, \\
& p_{m_{\min }} \leq p_{m} \leq p_{m_{\max }}, 1 \leq m \leq M .
\end{array}
$$

In the single-target case, the optimization (25) is identical to the problem $\mathbb{P} 5$. Similar to the multi-target case, the problem $\mathbb{P} 5$ is equivalent to the optimization problem $\mathbb{P} 6$ :

$$
\begin{aligned}
& \min _{\mathbf{p}, w} \mathbf{1}^{\mathrm{T}} \mathbf{p},
\end{aligned}
$$

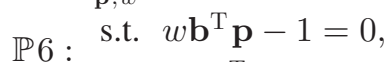

$$
\begin{aligned}
& w \eta \mathbf{p}^{\mathrm{T}} \mathbf{A} \mathbf{p}-1=0, \\
& p_{m_{\text {min }}} \leq p_{m} \leq p_{m_{\text {max }}}, 1 \leq m \leq M \text {. }
\end{aligned}
$$

This problem is nonconvex due to its equality constraint.

1) OCD-based method: The Lagrangian of (82) is

$L(\mathbf{p}, w, \lambda, \mu)=\mathbf{1}^{\mathrm{T}} \mathbf{p}+\lambda\left(w \mathbf{b}^{\mathrm{T}} \mathbf{p}-1\right)+\mu\left(w \eta \mathbf{p}^{\mathrm{T}} \mathbf{A} \mathbf{p}-1\right)$,

where $\lambda$ and $\mu$ are the dual variables. The gradients of this 419 Lagrangian are given by

$$
\begin{aligned}
& \Delta \mathbf{p}=\nabla_{\mathbf{p}} L(\mathbf{p}, w, \lambda, \mu)=\mathbf{1}+\lambda(w \mathbf{b})+\mu w \eta\left(\mathbf{A}+\mathbf{A}^{\mathrm{T}}\right) \mathbf{p} \\
& \Delta \lambda=\nabla_{-\lambda} L(\mathbf{p}, w, \lambda, \mu)=-w \mathbf{b}^{\mathrm{T}} \mathbf{p}+1 \\
& \Delta w=\nabla_{w} L(\mathbf{p}, w, \lambda, \mu)=\lambda \mathbf{b}^{\mathrm{T}} \mathbf{p}+\mu \eta \mathbf{p}^{\mathrm{T}} \mathbf{A} \mathbf{p} \\
& \Delta \mu=\nabla_{-\mu} L(\mathbf{p}, w, \lambda, \mu)=-\eta w \mathbf{p}^{\mathrm{T}} \mathbf{A} \mathbf{p}-1
\end{aligned}
$$

Given $\lambda^{(0)}, \mu^{(0)}$ and

$$
\mathbf{p}^{(0)}=\mathbf{p}_{e q u}=\frac{1}{\eta} \frac{\mathbf{b}^{\mathrm{T}} \mathbf{1}}{\mathbf{1}^{\mathrm{T}} \mathbf{A} \mathbf{1}} \mathbf{1},
$$

$\mathbf{p}, \lambda, w, \mu$ are updated in the following iterative procedure

$$
\begin{aligned}
p_{m}^{(n+1)} & =\left[p_{m}^{(n)}-\kappa_{1} \Delta p_{m}^{(n)}\right]_{p_{m_{\min }}}^{p_{m_{\max }}}, 1 \leq m \leq M, \\
\lambda^{(n+1)} & =\lambda^{(n)}-\kappa_{2} \Delta \lambda^{(n)}, \\
w^{(n+1)} & =w^{(n)}-\kappa_{3} \Delta w^{(n)}, \\
\mu^{(n+1)} & =\mu^{(n)}-\kappa_{4} \Delta \mu^{(n)},
\end{aligned}
$$

where again the step sizes are chosen according to (42). The 423 iterative procedure is repeated until $\left\|\mathbf{p}^{(n+1)}-\mathbf{p}^{(n)}\right\|$ becomes 424 smaller than a preset threshold.

2) ADMM-based method: Similar to the multi-target case, 426 we reformulate the problem $\mathbb{P} 6$ as

$$
\begin{aligned}
\min _{\mathbf{p}, \mathbf{z}} & \mathbf{1}^{\mathrm{T}} \mathbf{p}, \\
\text { s.t. } & \eta \mathbf{z}^{\mathrm{T}} \mathbf{A} \mathbf{p}-\mathbf{b}^{\mathrm{T}} \mathbf{p}=0, \\
& \mathbf{z}=\mathbf{p}, \\
& p_{m_{\text {m }}} \leq p_{m} \leq p_{m_{\text {max }}}, 1 \leq m \leq M .
\end{aligned}
$$

Then, by introducing an augmented Lagrangian, we have

$$
\begin{aligned}
\max _{\mathbf{e}, \mu} \min _{\mathbf{p}, \mathbf{z}} & \mathbf{1}^{\mathrm{T}} \mathbf{p}+\frac{\rho_{0}}{2}\|\mathbf{p}-\mathbf{z}+\mathbf{e}\|^{2} \\
& +\frac{\rho_{1}}{2}\left\|\eta \mathbf{z}^{\mathrm{T}} \mathbf{A} \mathbf{p}-\mathbf{b}^{\mathrm{T}} \mathbf{p}+\mu\right\|^{2}, \\
\text { s.t. } & p_{m_{\min }} \leq p_{m} \leq p_{m_{\max }}, 1 \leq m \leq M .
\end{aligned}
$$

With the initialization of $\mathbf{p}^{(0)}=\mathbf{z}^{(0)}=\mathbf{p}_{e q u}, \mathbf{e}^{(0)}=\mathbf{1}, \mu^{(0)}=429$ 1 , and $\rho_{0}^{(0)}$ and $\rho_{1}^{(0)}$ set to a large positive number, each iteration 430 involves the following steps. 
TABLE I

COMPLEXITy PER ITERATION OF THE OCD-BASED ALGORITHM

\begin{tabular}{|l|r|}
\hline Operation & Flops per iteration \\
\hline \hline Update $\mathbf{p}$ & $3 K M^{2}+(3 K+5) M+3 K$ \\
\hline Update $\lambda$ & $2 K M+2 K+2$, \\
\hline Update $\mathbf{w}$ & $2 K M^{2}+3 K M+5 K$ \\
\hline Update $\boldsymbol{\mu}$ & $2 K M^{2}+K M+4 K$ \\
\hline Total & $7 K M^{2}+(9 K+5) M+14 K+2$ \\
\hline
\end{tabular}

TABLE II

COMPLEXITY PER ITERATION OF THE ADMM-BASED AlgorithM

\begin{tabular}{|l|r|}
\hline Operation & Flops per iteration \\
\hline \hline Update $\mathbf{p}$ & $M^{3}+(5 K+7) M^{2}+(4 K+8) M+3 K+5$ \\
\hline Update $\mathbf{w}$ & $4 K M^{2}+\left(2 K^{2}+4 K\right) M+K^{2}+14 K$ \\
\hline Update $\mathbf{z}$ & $M^{3}+(7 K+2) M^{2}+(2 K+3) M+4 K$ \\
\hline Update $\mathbf{e}$ & $2 M$ \\
\hline Update $\mu$ & $2 K M+2 K+1$ \\
\hline Update $\gamma$ & $2 K M^{2}+K M+3 K$ \\
\hline Total & $2 M^{3}+(18 K+9) M^{2}+\left(2 K^{2}+13 K+13\right) M$ \\
\hline & $+K^{2}+26 K+6$ \\
\hline
\end{tabular}

- Step 1: Update $\mathbf{p}$. Isolating all the terms involving $\mathbf{p}$, the optimization is a constrained convex problem, leading to

$$
\begin{aligned}
& \overline{\mathbf{p}}^{(n+1)} \\
& \quad=\left(\rho_{0}^{(n)} \mathbf{I}_{M}+\rho_{1}^{(n)}\left(\eta \mathbf{A}^{\mathrm{T}} \mathbf{z}^{(n)}-\mathbf{b}\right)\right. \\
& \left.\quad \times\left(\eta\left(\mathbf{z}^{(n)}\right)^{\mathrm{T}} \mathbf{A}-\mathbf{b}^{\mathrm{T}}\right)\right)^{-1}\left(-\mathbf{1}+\rho_{0}^{(n)}\left(\mathbf{z}^{(n)}-\mathbf{e}^{(n)}\right)\right. \\
& \left.\quad-\rho_{1}^{(n)} \mu^{(n)}\left(\eta \mathbf{A}^{\mathrm{T}} \mathbf{z}^{(n)}-\mathbf{b}\right)\right) \\
& p_{m}^{(n+1)}=\left[\bar{p}_{m}^{(n+1)}\right]_{p_{m_{\text {m }} \text { in }}}^{p_{m_{\text {max }}}}, 1 \leq m \leq M .
\end{aligned}
$$

- Step 2: Update $\mathbf{z}$. Isolating all the terms involving $\mathbf{z}$, the problem is an unconstrained convex problem, leading to

$$
\begin{aligned}
\mathbf{z}^{(n+1)}= & \left(\rho_{0}^{(n)} \mathbf{I}_{M}+\rho_{1}^{(n)}\left(\eta \mathbf{A} \mathbf{p}^{(n+1)}\right)\left(\eta \mathbf{A} \mathbf{p}^{(n+1)}\right)^{\mathrm{T}}\right)^{-1} \\
& \times\left(\rho_{0}^{(n)}\left(\mathbf{p}^{(n+1)}+\mathbf{e}^{(n)}\right)\right. \\
& \left.+\rho_{1}^{(n)} \eta \mathbf{A} \mathbf{p}^{(n+1)}\left(\mathbf{b}^{\mathrm{T}} \mathbf{p}^{(n+1)}-\mu^{(n)}\right)\right) .
\end{aligned}
$$

- Step 3: Update e and $\mu$. The dual variables are updated according to

$$
\begin{aligned}
& \mu^{(n+1)}=\mu^{(n)}+\eta\left(\mathbf{z}^{(n+1)}\right)^{\mathrm{T}} \mathbf{A} \mathbf{p}^{(n+1)}-\mathbf{b}^{\mathrm{T}} \mathbf{p}^{(n+1)}, \\
& \mathbf{e}^{(n+1)}=\mathbf{e}^{(n)}+\mathbf{p}^{(n+1)}-\mathbf{z}^{(n+1)} .
\end{aligned}
$$

- Step 4: Update the $\rho_{0}$ and $\rho_{1}$ at the first a few iterations. By defining the primal and dual residuals $r_{0}^{(n+1)}$ and $s_{0}^{(n+1)}$ as

$$
\begin{aligned}
& r_{0}^{(n+1)}=\left\|\mathbf{p}^{(n+1)}-\mathbf{z}^{(n+1)}\right\|, \\
& s_{0}^{(n+1)}=\left\|\rho_{0}^{(n)}\left(\mathbf{z}^{(n)}-\mathbf{z}^{(n+1)}\right)\right\|,
\end{aligned}
$$

the updated $\rho_{0}^{(n+1)}$ is given by (75), and the dual variable $\mathbf{e}^{(n+1)}$ is rescaled according to (76). Similarly, define the primal and dual residuals $r_{1}^{(n+1)}$ and $s_{1}^{(n+1)}$ as

441 442 443

$$
\begin{aligned}
r_{1}^{(n+1)}= & \left|\eta\left(\mathbf{z}^{(n+1)}\right)^{\mathrm{T}} \mathbf{A} \mathbf{p}^{(n+1)}-\mathbf{b}^{\mathrm{T}} \mathbf{p}^{(n+1)}\right|, \quad \text { (102) } \\
s_{1}^{(n+1)}= & \| \mu^{(n+1)} \rho_{1}^{(n)} \eta \mathbf{A}^{\mathrm{T}}\left(\mathbf{z}^{(n)}-\mathbf{z}^{(n+1)}\right)+\rho_{1}^{(n)} \eta \\
& \times\left(\eta \mathbf{A}^{\mathrm{T}} \mathbf{z}^{(n)}-\mathbf{b}\right)\left(\mathbf{z}^{(n)}-\mathbf{z}^{(n+1)}\right)^{\mathrm{T}} \mathbf{A} \mathbf{p}^{(n+1)} \| .
\end{aligned}
$$

The updated $\rho_{1}^{(n+1)}$ is given by (77), and the rescaled dual 444 variable $\mu^{(n+1)}$ is given by (78). 445

3) A closed-form approximate solution: An equivalent La- 446 grangian associated with the problem $\mathbb{P} 5$ is $L(\mathbf{p}, \lambda)=\mathbf{1}^{\mathrm{T}} \mathbf{p}+447$ $\lambda\left(\eta \mathbf{p}^{\mathrm{T}} \mathbf{A} \mathbf{p}-\mathbf{b}^{\mathrm{T}} \mathbf{p}\right)$, whose KKT conditions are

$$
\begin{array}{r}
\mathbf{1}+\lambda\left(\eta\left(\mathbf{A}+\mathbf{A}^{\mathrm{T}}\right) \mathbf{p}-\mathbf{b}\right)=\mathbf{0}, \\
\eta \mathbf{p}^{\mathrm{T}} \mathbf{A} \mathbf{p}-\mathbf{b}^{\mathrm{T}} \mathbf{p}=\mathbf{0} .
\end{array}
$$

The authors of [12] obtained the closed-form optimal solution 449 $\lambda^{\star}$ and $\mathbf{p}^{\star}$ by jointly solving the two equations (104) and (105). 450 In particular, they calculated $\overline{\mathbf{p}}^{\star}$ from (104) as

$$
\overline{\mathbf{p}}^{\star}=\frac{\left(\mathbf{A}+\mathbf{A}^{\mathrm{T}}\right)^{-1}}{\eta}\left(\mathbf{b}-\frac{1}{\lambda^{\star}} \mathbf{1}\right),
$$

and then obtained $\mathbf{p}^{\star}$ by imposing the power constraints

$$
p_{m}^{\star}=\left[\bar{p}_{m}^{\star}\right]_{p_{m_{\text {m }} \text { in }}}^{p_{\text {max }}}, 1 \leq m \leq M .
$$

Unfortunately, this closed-form 'optimal' solution is generally invalid because in general $\mathbf{A}+\mathbf{A}^{\mathrm{T}}$ is not invertible.

Lemma 2: The rank of $\mathbf{A}+\mathbf{A}^{\mathrm{T}}$ is no more than 3 .

Proof:

$$
\begin{aligned}
\operatorname{rank}\left(\mathbf{A}+\mathbf{A}^{\mathrm{T}}\right) \leq & \operatorname{rank}\left(\mathbf{a}_{1,1}\left(\mathbf{a}_{2,2}\right)^{\mathrm{T}}-\mathbf{a}_{1,2}\left(\mathbf{a}_{2,1}\right)^{\mathrm{T}}\right. \\
& \left.+\mathbf{a}_{2,2}\left(\mathbf{a}_{1,1}\right)^{\mathrm{T}}-\mathbf{a}_{2,1}\left(\mathbf{a}_{1,2}\right)^{\mathrm{T}}\right) \\
\leq & \operatorname{rank}\left(\mathbf{a}_{1,1}\left(\mathbf{a}_{2,2}\right)^{\mathrm{T}}\right)+\operatorname{rank}\left(\mathbf{a}_{1,2}\left(\mathbf{a}_{2,1}\right)^{\mathrm{T}}\right) \\
& +\operatorname{rank}\left(\mathbf{a}_{2,2}\left(\mathbf{a}_{1,1}\right)^{\mathrm{T}}\right) \leq 3 .
\end{aligned}
$$

The second inequality is due to the fact that $\mathbf{a}_{1,2}=\mathbf{a}_{2,1}$.

Clearly, for any system with more than 3 transmit radars, the solution of (106) is invalid, and the minimum eigenvalue $\xi_{\min }$ of $\mathbf{A}+\mathbf{A}^{\mathrm{T}}$ is negative. We propose an approximate closed-form solution by replacing the invalid $\left(\mathbf{A}+\mathbf{A}^{\mathrm{T}}\right)^{-1}$ in (106) by the valid regularized form

$$
\mathbf{B}=\left(\mathbf{A}+\mathbf{A}^{\mathrm{T}}+\left(\left|\xi_{\min }\right|+\epsilon\right) \mathbf{I}_{M}\right)^{-1},
$$

where $\epsilon$ is a small positive number, such as, $\epsilon=0.0001$. Thus the 'unconstrained' power allocation is given as

$$
\overline{\mathbf{p}}^{*}=\frac{\mathbf{B}}{\eta}\left(\mathbf{b}-\frac{1}{\lambda^{*}} \mathbf{1}\right),
$$

457

458 459 460 461 462 
TABLE III

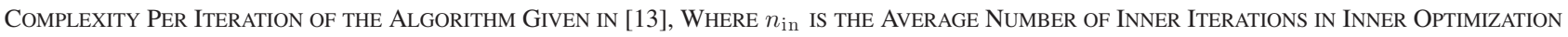
PROCEDURE

\begin{tabular}{|l|r|}
\hline Operation & Flops per inner iteration \\
\hline \hline Update the parameters of inner QCLP problem & $\left(5 M^{2}+2 M+1\right) / n_{\text {in }}$ \\
\hline Solve the inner QCLP problem & $M^{3}+(4 K+3) M^{2}+(6 K+10) M-K$ \\
\hline Total & $M^{3}+\left(4 K+3+\frac{5}{n_{\text {in }}}\right) M^{2}+\left(6 K+10+\frac{2}{n_{\text {in }}}\right) M-K$ \\
\hline
\end{tabular}

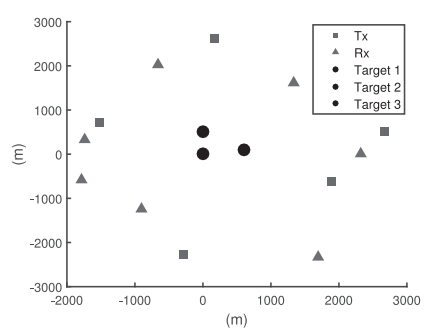

(a)

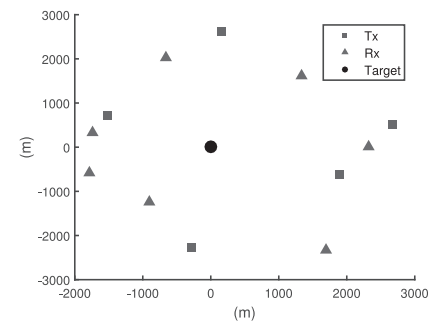

(b)

Fig. 2. Illustration of the MIMO radar system for: (a) three-target application, and (b) single-target application.

where $\lambda^{*}$ is obtained by substituting $\overline{\mathbf{p}}^{*}$ into (105) and taking the positive solution as

$$
\lambda^{*}=\frac{-b+\sqrt{b^{2}-4 a c}}{2 a},
$$

with

$$
\left\{\begin{array}{l}
a=\mathbf{b}^{\mathrm{T}} \mathbf{B}^{\mathrm{T}} \mathbf{A} \mathbf{B} \mathbf{b}-\mathbf{b}^{\mathrm{T}} \mathbf{B} \mathbf{b}, \\
b=-2 \mathbf{1}^{\mathrm{T}} \mathbf{B}^{\mathrm{T}} \mathbf{A}^{\mathrm{T}} \mathbf{B} \mathbf{b}^{\mathrm{T}}+2 \mathbf{b}^{\mathrm{T}} \mathbf{B} \mathbf{1}, \\
c=\mathbf{1}^{\mathrm{T}} \mathbf{B}^{\mathrm{T}} \mathbf{A} \mathbf{B} \mathbf{1}-\mathbf{1}^{\mathrm{T}} \mathbf{B} \mathbf{1} .
\end{array}\right.
$$

The solution $\mathbf{p}^{*}$ is then obtained by projecting $\overline{\mathbf{p}}^{*}$ onto the feasible region. This closed-form solution is inferior to the OCDbased and ADMM-based solutions in terms of its achievable performance, owing to its suboptimal nature.

\section{CONVERGENCE AND COMPLEXITy ANALysis}

Recall from Section II and III that our optimization problem $\mathbb{P} 1$ of (24) is nonconvex, and both our ADMM and OCD algorithms are based on a Lagrangian function approach. It is widely acknowledged that the zero duality gap cannot be guaranteed for general nonconvex problems. However, Yu and Lui [24] proposed a theorem which guarantees the zero duality gap for the nonconvex problem that meets the 'time-sharing condition'. In Appendix B, we proved that our optimization problem $\mathbb{P} 1$ satisfies the time-sharing condition of [24]. Hence, the strong duality holds for $\mathbb{P} 1$. We are now ready to prove that both our two algorithms can converge to a local optimal point under some assumptions.

\section{A. Convergence of the Proposed Algorithms}

1) The ADMM-based algorithm: We first point out again that since our problem is nonconvex, the ADMM-based algorithm can only guarantee to converge to a local optimal solution. The convergence of the ADMM method is proved for the convex optimization problem in [18], while Magnússon et al. 490 [25] extended the convergence results to the nonconvex case. 491 The convergence of our ADMM-based algorithm will be fur- 492 ther illustrated in Section $\mathrm{V}$ using simulations.

2) The OCD-based algorithm: Again, since our optimization problem is nonconvex, the OCD-based algorithm can only find a locally optimal solution. Collect all the primal variables of the Lagrangian (32) together as $\mathbf{y}=\left[\mathbf{p}^{\mathrm{T}} \mathbf{w}^{\mathrm{T}}\right]^{\mathrm{T}}$ and denote the cost function and the constraints of $\mathbb{P} 3$ respectively by

$$
\begin{aligned}
f(\mathbf{y}) & =\mathbf{1}^{\mathrm{T}} \mathbf{p}, \\
g_{0}(\mathbf{y}) & =\sum_{k=1}^{K} v_{k} w_{k} \mathbf{b}_{k}^{\mathrm{T}} \mathbf{p}-1, \\
g_{k}(\mathbf{y}) & =w_{k} \eta \mathbf{p}^{\mathrm{T}} \mathbf{A}_{k} \mathbf{p}-1,1 \leq k \leq K .
\end{aligned}
$$

According to Theorem 2 in Section 8.2.3 and Lemma 5 in 499 Section 2.1 of [26], to prove the convergence of the OCD al- 500 gorithm, we have to prove that the second derivatives $\nabla^{2} f(\mathbf{y}) \quad 501$ and $\nabla^{2} g_{k}(\mathbf{y})$ for $0 \leq k \leq K$ satisfy the Lipschitz condition in 502 a neighbourhood of the optimal primal point $\mathbf{y}^{\star}$. Note that 503

$$
\nabla^{2} f(\mathbf{y})=\mathbf{0},
$$

$$
\nabla^{2} g_{0}(\mathbf{y})=\left[\begin{array}{cc}
\mathbf{0} & v_{1} \mathbf{b}_{1} \cdots v_{K} \mathbf{b}_{K} \\
v_{1} \mathbf{b}_{1}^{\mathrm{T}} & \\
\vdots & \mathbf{0} \\
v_{K} \mathbf{b}_{K}^{\mathrm{T}} &
\end{array}\right]
$$

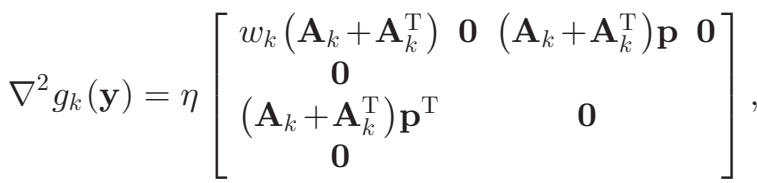

$$
\begin{aligned}
& 1 \leq k \leq K .
\end{aligned}
$$

Since $\nabla^{2} f(\mathbf{y})$ and $\nabla^{2} g_{0}(\mathbf{y})$ are constants, they satisfy the 504 required Lipschitz condition. For $\mathbf{p}_{\min } \leq \mathbf{p} \leq \mathbf{p}_{\max }$, all the el- 505 ements in the matrix $\nabla^{2} g_{k}(\mathbf{y})$, where $1 \leq k \leq K$, are finite. 506 Therefore, it is easy to find a constant $\varsigma$ satisfying

$$
\left\|\nabla^{2} g_{k}\left(\mathbf{y}_{1}\right)-\nabla^{2} g_{k}\left(\mathbf{y}_{2}\right)\right\| \leq \varsigma\left\|\mathbf{y}_{1}-\mathbf{y}_{2}\right\| .
$$

Thus $\nabla^{2} g_{k}(\mathbf{y})$ satisfies the required Lipschitz condition too. $\quad 508$

According to [26], under the assumption that the Hessian ma- 509 trix of the Lagrangian (32) with respect to $y$ at the minimum pri- 510 mal point $\mathbf{y}^{\star}=\left(\mathbf{p}^{\star}, \mathbf{w}^{\star}\right)$ is positive definite, the Hessian matrix 511 of the Lagrangian (32) with respect to the primal and dual vari- 512 ables is negative definite at the optimal point $\left(\mathbf{p}^{\star}, \mathbf{w}^{\star}, \lambda^{\star}, \boldsymbol{\mu}^{\star}\right) .513$ Then there exists a positive number $\bar{\kappa}=\min _{i}-\Re\left[\bar{\xi}_{i}\right]\left|\bar{\xi}_{i}\right|^{-2}, \quad 514$ 
TABLE IV

SYSTEM PARAMETERS

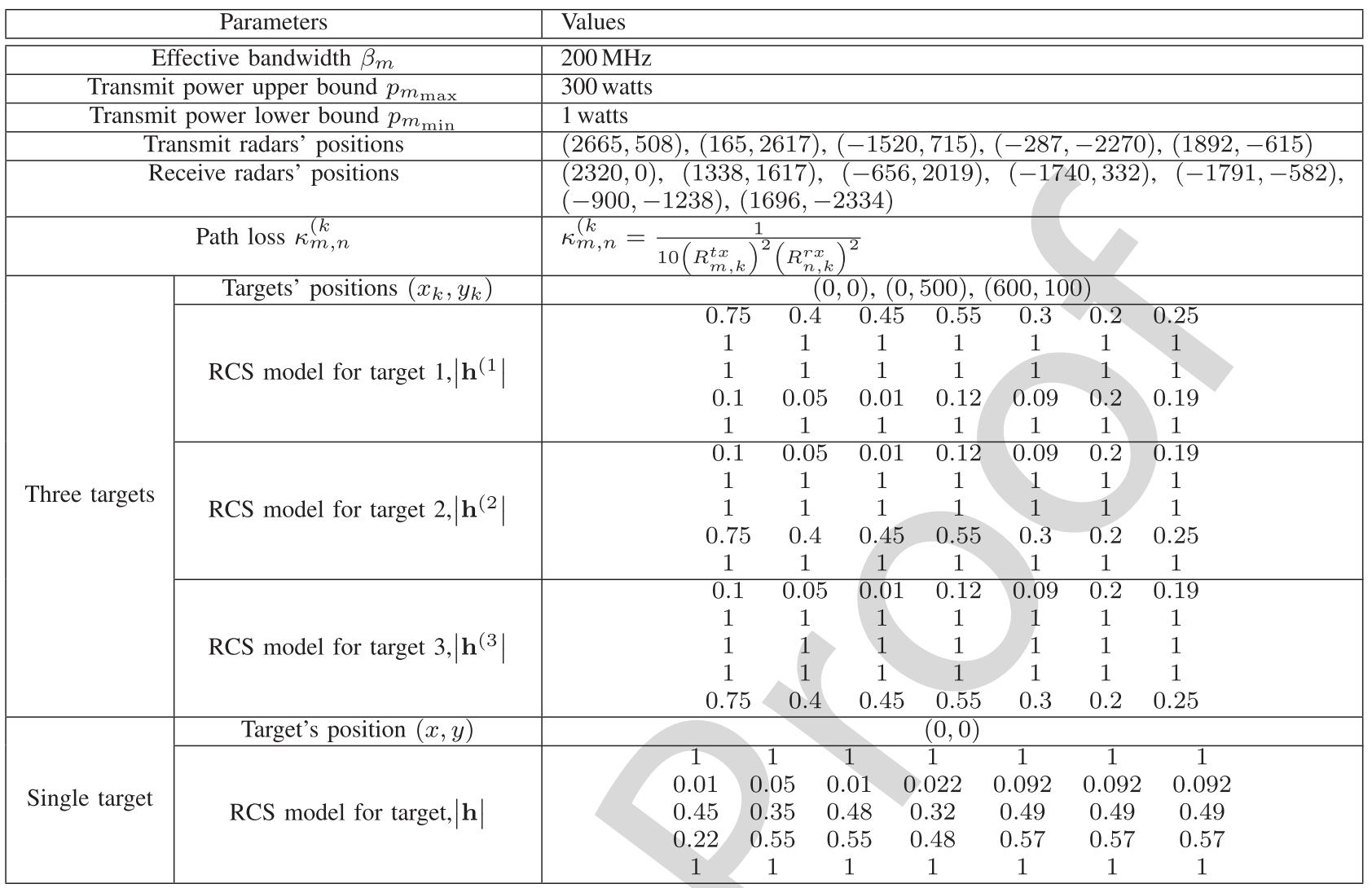

where $\bar{\xi}_{i}$ are the eigenvalues of the Hessian matrix of the Lagrangian (32) with respect to the primal and dual variables at $\left(\mathbf{p}^{\star}, \mathbf{w}^{\star}, \lambda^{\star}, \boldsymbol{\mu}^{\star}\right)$. Consequently, as long as the maximum of the four step sizes $\kappa_{\max }=\max _{1 \leq i \leq 4} \kappa_{i}$ satisfies the condition of $\kappa_{\max } \leq \bar{\kappa}$, our scheme (37)-(40) will converge to the locally optimal point $\left(\mathbf{p}^{\star}, \mathbf{w}^{\star}, \lambda^{\star}, \boldsymbol{\mu}^{\star}\right)$ when starting from a neighbourhood of $\left(\mathbf{p}^{\star}, \mathbf{w}^{\star}, \lambda^{\star}, \boldsymbol{\mu}^{\star}\right)$, according to [26]. In practice, $\bar{\kappa}$ is unknown. It is advisable to choose sufficiently small step sizes $\kappa_{i}, 1 \leq i \leq 4$, in order to ensure the convergence of the OCD scheme.

Remark 4: A positive-definite Hessian matrix of the Lagrangian (32) with respect to $\mathbf{y}$ at $\mathbf{y}^{\star}$ is a sufficient condition for the convergence of the OCD scheme. If this Hessian matrix is semi-positive definite, we cannot prove the convergence of the OCD scheme based on the result of [26]. By adopting an exponentially decaying step size $\kappa_{\max } \propto e^{-\alpha n}$, we ensure that our OCD algorithm works well in any situation.

\section{B. Complexity of Proposed Algorithms and Algorithm of [13]}

The complexity of our OCD and ADMM algorithms are summarized in Tables I and II, respectively. For the ADMM-based algorithm, since the penalty parameters are only updated in the first few iterations, the complexity associated with this part of operation is omitted. Additionally, we assume that GaussJordan elimination is used for matrix inversion and, therefore, the number of flops required by inverting an $M \times M$ matrix is $M^{3}+M^{2}+M$. For the OCD-based algorithm, the complexity of computing the four step sizes is negligible and therefore it 541 is also omitted. Clearly, the complexity of the ADMM-based 542 algorithm is on the order of $M^{3}$ per iteration, which is denoted 543 by $\mathrm{O}\left(M^{3}\right)$, while the complexity of the OCD-based algorithm 544 is on the order of $\mathrm{O}\left(M^{2}\right)$ per iteration. It will be shown by our 545 simulation results that the convergence speed of the ADMM al- 546 gorithm is at least one order of magnitude faster than that of the 547 OCD algorithm. Therefore, despite its higher per-iteration com- 548 plexity, the ADMM algorithm actually imposes a lower total 549 complexity, compared to the OCD algorithm.

The benchmark scheme of [13] invokes two iterative loops for solving the optimization problem (25). Specifically, at each outer iteration, the parameters of the inner quadratic constrained linear programming (QCLP) problem are updated, and the QCLP problem is then solved iteratively in the inner iterative loop. We assume that the interior-point method is used for solving this inner QCLP, which requires $n_{\text {in }}$ iterations on average. Based on the above discussions, the complexity of the algorithm of [13] is summarized in Table III, where it is seen that the complexity per inner iteration is on the order of $\mathrm{O}\left(M^{3}\right)$. Thus the complexity of our ADMM-based algorithm is only marginally higher than that of the algorithm in [13], because they are both on the order of $\mathrm{O}\left(M^{3}\right)$ per iteration. The algorithm of [13] requires a total of $n_{\mathrm{ou}} n_{\mathrm{in}}$ iterations to converge, where $n_{\mathrm{ou}}$ is the number of iterations for the outer iterative loop. As it will be shown in the simulation results, the number of iterations required for the ADMM-based algorithm to converge is very close to the total number of iterations $n_{\mathrm{ou}} n_{\text {in }}$ required by the algorithm of [13]. 


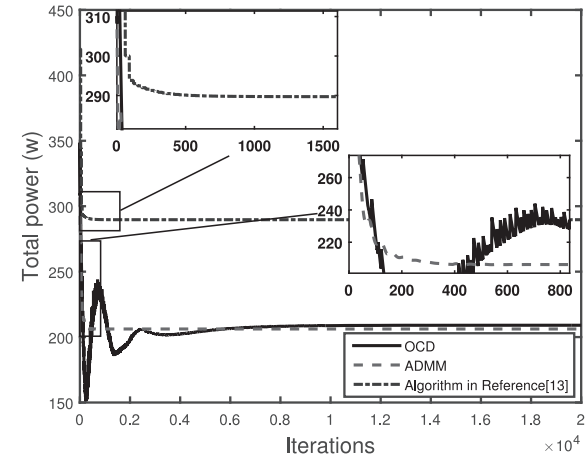

(a)

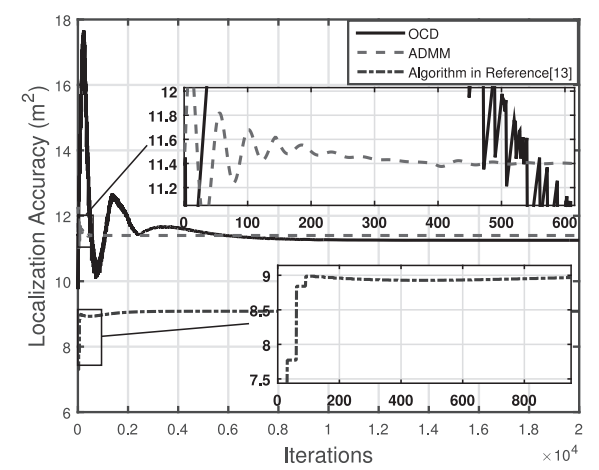

(b)

Fig. 3. Convergence performance of three algorithms, in terms of (a) total power consumption, and (b) aggregate localization accuracy, for the three-target case with $v_{1}=1, v_{2}=2$ and $v_{3}=1$.

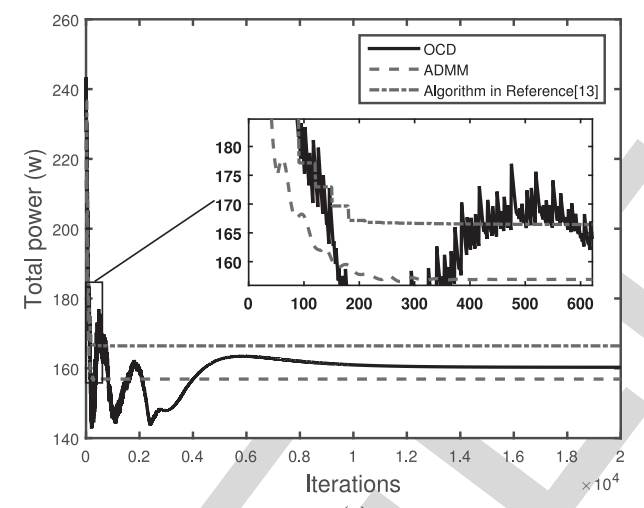

(a)

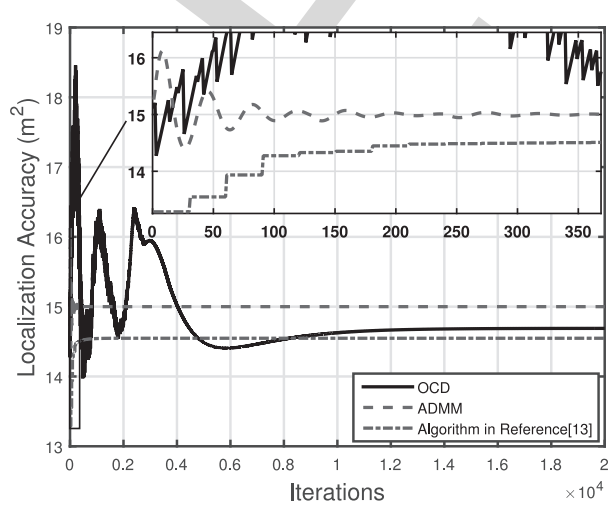

(b)

Fig. 4. Convergence performance of three algorithms, in terms of (a) total power consumption, and (b) aggregate localization accuracy, for the three-target case with $v_{1}=v_{2}=v_{3}=1$.
In this sense, both algorithms require a similar total complexity 569 for solving their associated optimization problems. Although 570 our OCD-based algorithm enjoys a much lower complexity per 571 iteration than the algorithm of [13], it imposes a higher total 572 complexity.

\section{Simulation Results}

Let us now evaluate the performance of the proposed al- 575 gorithms using a MIMO radar system having $M=5$ trans- 576 mit radars and $N=7$ receive radars. The algorithm of [13] is 577 used as the benchmark. Fig. 2 depicts both the triple-target and 578 single-target cases considered. The system parameters of both 579 the triple-target and single-target cases are listed in Table IV. The 580 localization accuracy threshold $\eta$ is set to $15 \mathrm{~m}^{2}$ for the triple- 581 target case and $10 \mathrm{~m}^{2}$ for the single-target case. The exponential 582 decaying factor is empirically chosen to be $\alpha=0.0005$ for the 583 four step sizes of the OCD algorithm.

\section{A. Triple-Target Case}

We consider the two sets of the importance weightings for the three targets given by: i) $v_{1}=1, v_{2}=2$ and $v_{3}=1$, and ii) $v_{1}=v_{2}=v_{3}=1$. For the sake of a fair comparison to the algorithm of [13], the effects of these weightings have to be taken into consideration, and the target estimation error thresholds for the three constraints of the optimization problem (25) are suitably scaled as

$$
\frac{\mathbf{b}_{1}^{\mathrm{T}} \mathbf{p}}{\mathbf{p}^{\mathrm{T}} \mathbf{A}_{1} \mathbf{p}} \leq \bar{\eta}_{1}, \frac{\mathbf{b}_{2}^{\mathrm{T}} \mathbf{p}}{\mathbf{p}^{\mathrm{T}} \mathbf{A}_{2} \mathbf{p}} \leq \bar{\eta}_{2}, \frac{\mathbf{b}_{3}^{\mathrm{T}} \mathbf{p}}{\mathbf{p}^{\mathrm{T}} \mathbf{A}_{3} \mathbf{p}} \leq \bar{\eta}_{3},
$$

with $\bar{\eta}_{1}=\frac{1}{3 v_{1}} \eta, \bar{\eta}_{2}=\frac{1}{3 v_{2}} \eta$ and $\bar{\eta}_{3}=\frac{1}{3 v_{3}} \eta$. For our ADMM algorithm, the initial values of the dual variables are set to $\mathbf{e}^{(0)}=\left[\begin{array}{llll}1 & 1 & 1 & 1\end{array}\right]^{\mathrm{T}}, \mu^{(0)}=1$ and $\gamma_{k}^{(0)}=1$ for $1 \leq k \leq 3$, while all the initial penalty parameters are set to 500 . For our OCD algorithm, the initial values of the dual variables are set to $\lambda^{(0)}=1$ and $\mu_{k}^{(0)}=1$ for $1 \leq k \leq 3$. Additionally, the four constants in the four step sizes of the OCD algorithm are set to $c_{1}=0.3, c_{2}=1.0, c_{3}=1.5$ and $c_{4}=1.1$ for the senario i), while they are set to $c_{1}=0.3, c_{2}=0.9, c_{3}=1.5$ and $c_{4}=1.1$ for the senario ii). These parameters were found empirically to be appropriate for the corresponding application scenarios. For the algorithm of [13], we use the CVX software to solve its inner QCLP problem. In our simulations, we observe that the CVX converges within 25 to 35 iterations. Therefore, we will assume that the average number of inner iterations for the algorithm of [13] is $n_{\text {in }}=30$.

Fig. 3 compares the total power allocations $\mathbf{p}$ and the aggregate localization accuracy results of $\sum_{k=1}^{3} \frac{\mathbf{b}_{k}^{\mathrm{T}} \mathbf{p}}{\mathbf{p}^{\mathrm{T}} \mathbf{A}_{k} \mathbf{p}}$ obtained by the three algorithms for the senario i), while Fig. 4 depicts the results for the senario ii). It can be seen that the number of iterations required by the ADMM-based algorithm to converge is similar to the total number of iterations $n_{\text {ou }} n_{\text {in }}$ required by the algorithm of [13], while the convergence speed of the OCDbased algorithm is considerably slower than that of the other two algorithms. As expected, our algorithms outperform the algorithm of [13] in terms of its total power consumption, albeit at the expense of some degradation in localization accuracy. 587 588 589 500 591 592 (n) . . (as 
TABLE V

PERFormance COMPARISON OF THREE AlgORITHMS FOR THE THREE-TARGET CASE

\begin{tabular}{|c|c|c|c|c|c|c|}
\hline & \multicolumn{3}{|c|}{ ii) $v_{1}=v_{2}=v_{3}=1$} & \multicolumn{3}{|c|}{ i) $v_{1}=1, v_{2}=2, v_{3}=1$} \\
\hline & ADMM & OCD & {$[13]$} & ADMM & OCD & {$[13]$} \\
\hline Radar 1: Power (watts) & 1 & 1 & 1 & 1 & 1 & 1 \\
\hline Radar 2: Power (watts) & 95.8 & 93.3 & 102 & 119.6 & 117.9 & 75.8 \\
\hline Radar 3: Power (watts) & 58.2 & 64.0 & 40.3 & 83.5 & 88.1 & 170.4 \\
\hline Radar 4: Power (watts) & 1 & 1 & 1 & 1 & 1 & 1 \\
\hline Radar 5: Power (watts) & 1 & 1 & 22.2 & 1 & 1 & 41.5 \\
\hline Total Power (watts) & 157 & 160.3 & 166.5 & 206.1 & 209.0 & 289.7 \\
\hline Target 1: Localization Accuracy $\left(\mathrm{m}^{2}\right)$ & 5.4 & 5.3 & 5 & 4.1 & 4.1 & 3.1 \\
\hline Target 2: Localization Accuracy $\left(\mathrm{m}^{2}\right)$ & 4.8 & 4.7 & 4.5 & 3.6 & 3.5 & 2.5 \\
\hline Target 3: Localization Accuracy $\left(\mathrm{m}^{2}\right)$ & 4.8 & 4.7 & 5 & 3.7 & 3.6 & 3.5 \\
\hline Aggregate Localization Accuracy $\left(\mathrm{m}^{2}\right)$ & 15 & 14.7 & 14.5 & 11.4 & 11.3 & 9.1 \\
\hline Total Power Saving & $5.7 \%$ & $3.7 \%$ & - & $28.9 \%$ & $27.9 \%$ & - \\
\hline Degradation in Aggregate Localization Accuracy & $3.4 \%$ & $1.4 \%$ & - & $25.3 \%$ & $27.9 \%$ & \\
\hline Average Total Power Saving & $10.0 \%$ & $10.5 \%$ & - & $20.0 \%$ & $25.6 \%$ & - \\
\hline Average Degradation in Aggregate Localization Accuracy & $8.6 \%$ & $8.9 \%$ & - & $27.2 \%$ & $30.0 \%$ & - \\
\hline
\end{tabular}

The average results are obtained over 1000 random simulation experiments.

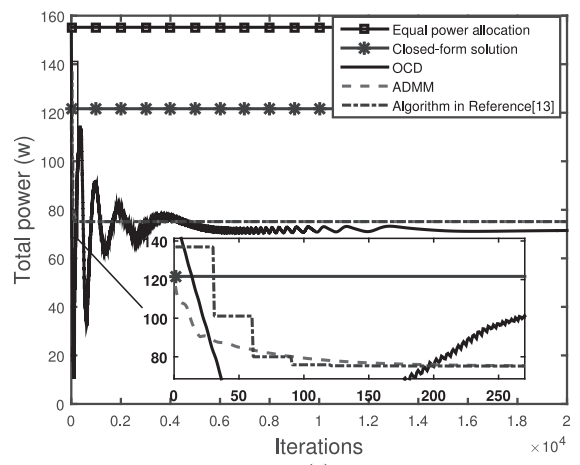

(a)

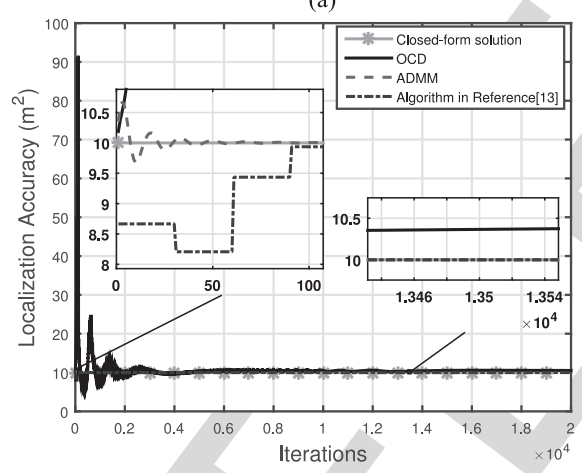

(b)

Fig. 5. Convergence performance of three algorithms, in terms of (a) total power consumption, and (b) aggregate localization accuracy, in comparison with the EPA and the closed-form solution, for the single-target case.

Table $\mathrm{V}$ details how our algorithms trade the localization accuracy against the transmit power, in comparison to the algorithm of [13]. Specifically, for the senario of i), our ADMM algorithm achieves $28.9 \%$ power saving at the cost of $25.3 \%$ degradation in aggregate localization accuracy, while our OCD algorithm trades $27.9 \%$ power saving against $27.9 \%$ degradation in localization accuracy. For the equal weighting senario of ii), the savings in power achieved by our two algorithms are considerably smaller but the losses in localization accuracy are also significantly smaller, compared with the senario i). To obtain statistically relevant comparison, we carry out 1000 simulations by randomly locating all the transmit radars and receive radars at 631 the radius $R=3000\left(0.5+\varepsilon_{x}\right)$ m with the angular rotations of 632 $\theta=2 \pi \varepsilon_{y}$, where $\varepsilon_{x}$ and $\varepsilon_{y}$ are uniformly distributed in $[0,1.0] .633$ The average power saving and degradation in localization accu- 634 racy over the 1000 random experiments are listed in the last two 635 rows of Table $\mathrm{V}$.

\section{B. Single-Target Case}

The four constants in the four step sizes of the OCD algorithm are set to $c_{1}=c_{2}=1.0$ and $c_{3}=c_{4}=0.3$, which is empirically found to be appropriate for this application senario. Fig. 5 characterizes the performance of our ADMM-based and OCD-based algorithms as well as the algorithm of [13]. As expected, all the three algorithms attain the same performance, both in terms of total power allocated and localization accuracy, since the underlying optimization problems are identical in the single-target case. In terms of convergence speed, our ADMM-based algorithm outperforms the algorithm of [13], while the OCD-based algorithm is considerably slower than the algorithm of [13]. In Fig. 5 (a), we also characterize the equalpower allocation (EPA) scheme and the closed-form solution of SubSection III-B3. It can be seen that our closed-form solution performs significantly better than the EPA scheme, but it is inferior to the other three iterative algorithms because the suboptimal nature of this closed-form solution.

\section{CONCLUSION}

The target localization problem of distributed MIMO radar systems has been investigated, which minimizes the power of the transmit radars, while meeting a required localization accuracy. We have proposed the OCD-based and ADMM-based iterative algorithms to solve this nonconvex optimization problem. Both the algorithms are capable of converging to a local optimum. The OCD algorithm imposes a much lower computational complexity per iteration, while the ADMM algorithm achieves a much faster convergence. For the multi-target senario, we have shown how our proposed approach trades the power saving with some degradation in localization accuracy, 
compared with that of state-of-the-art scheme [13]. We have also demonstrated that our ADMM-based algorithm and the existing state-of-the-art scheme have similar computational complexity and convergence speed. For the single-target senario, we have confirmed that our algorithms and the benchmark attain the same performance in terms of both power consumption and localization accuracy, because the underlying optimization problems become identical.

\section{APPENDIX}

\section{A. Derivation of Updating Formulae for Penalty Parameters}

The optimal solution to the $\mathbb{P} 4$ of (45) should be primal and dual feasible, that is,

$$
\begin{aligned}
& \mathbf{p}^{(n+1)}-\mathbf{z}^{(n+1)}=\mathbf{0}, \\
& \sum_{k=1}^{K} w_{k}^{(n+1)} v_{k} \mathbf{b}_{k}^{\mathrm{T}} \mathbf{p}^{(n+1)}-1=0, \\
& w_{k}\left(\mathbf{z}^{(n+1)}\right)^{\mathrm{T}} \mathbf{A}_{k} \mathbf{p}^{(n+1)} \eta-1=0,1 \leq k \leq K, \\
& \frac{\partial L^{\prime}\left(\mathbf{p}, \mathbf{z}^{(n+1)}, \mathbf{w}^{(n+1)}, \mathbf{d}_{0}^{(n+1)}, d_{1}^{(n+1)}, \mathbf{d}_{2}^{(n+1)}\right)}{\partial \mathbf{p}}=\mathbf{0}, \\
& \frac{\partial L^{\prime}\left(\mathbf{p}^{(n+1)}, \mathbf{z}^{(n+1)}, \mathbf{w}, \mathbf{d}_{0}^{(n+1)}, d_{1}^{(n+1)}, \mathbf{d}_{2}^{(n+1)}\right)}{\partial \mathbf{w}}=\mathbf{0}, \\
& \frac{\partial L^{\prime}\left(\mathbf{p}^{(n+1)}, \mathbf{z}, \mathbf{w}^{(n+1)}, \mathbf{d}_{0}^{(n+1)}, d_{1}^{(n+1)}, \mathbf{d}_{2}^{(n+1)}\right)}{\partial \mathbf{z}}=\mathbf{0},
\end{aligned}
$$

where $L^{\prime}\left(\mathbf{p}, \mathbf{w}, \mathbf{z}, \mathbf{d}_{0}, d_{1}, \mathbf{d}_{2}\right)$ is the Lagrangian of (45), which can be separated into three parts

$$
\begin{aligned}
& L^{\prime}\left(\mathbf{p}, \mathbf{w}, \mathbf{z}, \mathbf{d}_{0}, d_{1}, \mathbf{d}_{2}\right)=\underbrace{\mathbf{1}^{\mathrm{T}} \mathbf{p}+\mathbf{d}_{0}^{\mathrm{T}}(\mathbf{p}-\mathbf{z})}_{L_{0}^{\prime}\left(\mathbf{p}, \mathbf{z}, \mathbf{d}_{0}\right)}+ \\
& \underbrace{d_{1}\left(\sum_{k=1}^{K} w_{k} v_{k} \mathbf{b}_{k}^{\mathrm{T}} \mathbf{p}-1\right)}_{L_{1}^{\prime}\left(\mathbf{p}, \mathbf{w}, d_{1}\right)}+\underbrace{\sum_{k=1}^{K} d_{2, k}\left(w_{k} \mathbf{z}^{\mathrm{T}} \mathbf{A}_{k} \mathbf{p} \eta-1\right)}_{L_{2}^{\prime}\left(\mathbf{p}, \mathbf{w}, \mathbf{z}, \mathbf{d}_{2}\right)} .
\end{aligned}
$$

1 However, the ADMM-based algorithm uses the augmented Lagrangian of

$$
\begin{gathered}
L\left(\mathbf{p}, \mathbf{w}, \mathbf{z}, \mathbf{d}_{0}, d_{1}, \mathbf{d}_{2}\right)=\underbrace{\mathbf{1}^{\mathrm{T}} \mathbf{p}+\frac{\rho_{0}}{2}\|\mathbf{p}-\mathbf{z}\|^{2}+\mathbf{d}_{0}^{\mathrm{T}}(\mathbf{p}-\mathbf{z})}_{L_{0}\left(\mathbf{p}, \mathbf{z}, \mathbf{d}_{0}\right)} \\
+\underbrace{\frac{\rho_{1}}{2}\left|\sum_{k=1}^{K} w_{k} v_{k} \mathbf{b}_{k}^{\mathrm{T}} \mathbf{p}-1\right|^{2}+d_{1}\left(\sum_{k=1}^{K} w_{k} v_{k} \mathbf{b}_{k}^{\mathrm{T}} \mathbf{p}-1\right)}_{L_{1}\left(\mathbf{p}, \mathbf{w}, d_{1}\right)} \\
+\underbrace{\sum_{k=1}^{K} \frac{\rho_{2, k}}{2}\left|w_{k} \mathbf{z}^{\mathrm{T}} \mathbf{A}_{k} \mathbf{p} \eta-1\right|^{2}+\sum_{k=1}^{K} d_{2, k}\left(w_{k} \mathbf{z}^{\mathrm{T}} \mathbf{A}_{k} \mathbf{p} \eta-1\right)}_{L_{2}\left(\mathbf{p}, \mathbf{w}, \mathbf{z}, \mathbf{d}_{2}\right)},
\end{gathered}
$$

which can be divided into three parts, and all the primal and 683 dual variables are updated one by one. Thus, in every iteration, 684 there exist primal and dual residuals.

Specifically, in the $(n+1)$ th iteration, the primal residuals 686 are given by $r_{0}^{(n+1)}$ of (65), $r_{1}^{(n+1)}$ of (66), and $r_{2, k}^{(n+1)}$ for 687 $1 \leq k \leq K$ of (67), while the dual residuals are defined via

$$
d r=\sqrt{\left\|\mathbf{d r}_{0}\right\|^{2}+\left\|\mathbf{d r}_{1}\right\|^{2}+\left\|\mathbf{d r}_{2}\right\|^{2}},
$$

with

$$
\begin{aligned}
\mathbf{d r}_{0}= & \frac{\partial L\left(\mathbf{p}, \mathbf{z}^{(n)}, \mathbf{w}^{(n)}, \mathbf{d}_{0}^{(n)}, d_{1}^{(n)}, \mathbf{d}_{2}^{(n)}\right)}{\partial \mathbf{p}} \\
& -\frac{\partial L^{\prime}\left(\mathbf{p}, \mathbf{z}^{(n+1)}, \mathbf{w}^{(n+1)}, \mathbf{d}_{0}^{(n+1)}, d_{1}^{(n+1)}, \mathbf{d}_{2}^{(n+1)}\right)}{\partial \mathbf{p}}, \\
\mathbf{d r}_{1}= & \frac{\partial L\left(\mathbf{p}^{(n+1)}, \mathbf{z}^{(n)}, \mathbf{w}, \mathbf{d}_{0}^{(n)}, d_{1}^{(n)}, \mathbf{d}_{2}^{(n)}\right)}{\partial \mathbf{w}} \\
& -\frac{\partial L^{\prime}\left(\mathbf{p}^{(n+1)}, \mathbf{z}^{(n+1)}, \mathbf{w}, \mathbf{d}_{0}^{(n+1)}, d_{1}^{(n+1)}, \mathbf{d}_{2}^{(n+1)}\right)}{\partial \mathbf{w}}, \\
\mathbf{d} \mathbf{r}_{2}= & \frac{\partial L\left(\mathbf{p}^{(n+1)}, \mathbf{z}, \mathbf{w}^{(n+1)}, \mathbf{d}_{0}^{(n)}, d_{1}^{(n)}, \mathbf{d}_{2}^{(n)}\right)}{\partial \mathbf{z}} \\
& -\frac{\partial L^{\prime}\left(\mathbf{p}^{(n+1)}, \mathbf{z}, \mathbf{w}^{(n+1)}, \mathbf{d}_{0}^{(n+1)}, d_{1}^{(n+1)}, \mathbf{d}_{2}^{(n+1)}\right)}{\partial \mathbf{z}} .
\end{aligned}
$$

It can be seen that the primal residuals $r_{0}^{(n+1)}, r_{1}^{(n+1)}$ and 690 $r_{2, k}^{(n+)}$ for $1 \leq k \leq K$ are related to $L_{0}\left(\mathbf{p}, \mathbf{z}, \mathbf{d}_{0}\right), L_{1}\left(\mathbf{p}, \mathbf{w}, d_{1}\right) \quad 691$ and $L_{2}\left(\mathbf{p}, \mathbf{w}, \mathbf{z}, \mathbf{d}_{2}\right)$, respectively. Therefore, we will similarly 692 'separate' the dual residuals into $s_{0}^{(n+1)}, s_{1}^{(n+1)}$ and $s_{2, k}^{(n+1)}$ for 693 $1 \leq k \leq K$, corresponding to $L_{0}\left(\mathbf{p}, \mathbf{z}, \mathbf{d}_{0}\right), L_{1}\left(\mathbf{p}, \mathbf{w}, d_{1}\right)$ and 694 $L_{2}\left(\mathbf{p}, \mathbf{w}, \mathbf{z}, \mathbf{d}_{2}\right)$, respectively.

In order to analyze the updating formula (75) for the penalty 696 parameter $\rho_{0}$, we have to calculate $s_{0}^{(n+1)}$ as follows $\quad 697$

$$
\begin{aligned}
s_{0}^{(n+1)}= & \left(\left\|\frac{\partial L_{0}\left(\mathbf{p}^{(n+1)}, \mathbf{z}, \mathbf{d}_{0}^{(n)}\right)}{\partial \mathbf{z}}-\frac{\partial L_{0}^{\prime}\left(\mathbf{p}^{(n+1)}, \mathbf{z}, \mathbf{d}_{0}^{(n+1)}\right)}{\partial \mathbf{z}}\right\|^{2}\right. \\
& \left.+\left\|\frac{\partial L_{0}\left(\mathbf{p}, \mathbf{z}^{(n)}, \mathbf{d}_{0}^{(n)}\right)}{\partial \mathbf{p}}-\frac{\partial L_{0}^{\prime}\left(\mathbf{p}, \mathbf{z}^{(n+1)}, \mathbf{d}_{0}^{(n+1)}\right)}{\partial \mathbf{p}}\right\|^{2}\right)^{\frac{1}{2}} .
\end{aligned}
$$

By evaluating the required four partial derivatives and plugging 698 them into (131), we arrive at the dual residual $s_{0}^{(n+1)}$ of (68). 699 Note that a large value for $\rho_{0}$ adds a large penalty on the violation 700 of primal feasibility and, therefore, a large $\rho_{0}$ reduces the primal 701 residual $r_{0}^{(n+1)}$. On the other hand, from the expression (68), it 702 is seen that a small $\rho_{0}$ reduces the dual residual $s_{0}^{(n+1)}$. Thus, 703 in order to balance the primal and dual residuals $r_{0}^{(n+1)}$ and 704 $s_{0}^{(n+1)}$, the penalty parameter $\rho_{0}$ is updated according to (75), 705 which is beneficial to convergence. 
707 Similarly, it can be shown that the dual residual $s_{1}^{(n+1)}$ re708 lated to $L_{1}\left(\mathbf{p}, \mathbf{w}, d_{1}\right)$ is given by (69) and (71), while the dual 709 residuals $s_{2, k}^{(n+1)}$ for $1 \leq k \leq K$ related to $L_{2}\left(\mathbf{p}, \mathbf{w}, \mathbf{z}, \mathbf{d}_{2}\right)$ are 710 specified by (70), (72) and (73). Following the same logic of 711 balancing the primal and dual residuals, the updating formulae 712 for the penalty parameters $\rho_{1}$ and $\rho_{2, k}$ are specified by (76) and 713 (78), respectively.

\section{4}

\section{B. Proof of the Time-Sharing Condition for Problem $\mathbb{P} 1$}

According to [24], the time-sharing condition for the optimization problem $\mathbb{P} 1$ of (24) is as follows. Time-sharing condition: Let $\mathbf{p}_{1}$ and $\mathbf{p}_{2}$ be the optimal solutions of $\mathbb{P} 1$ in conjunction with $\eta=\eta_{1}$ and $\eta=\eta_{2}$, respectively. $\mathbb{P} 1$ is said to satisfy the time-sharing condition if for any $\eta_{1}$ and $\eta_{2}$ and for any $0 \leq \xi \leq 1$, there always exists a feasible solution $\mathbf{p}_{3}$ so that $\sum_{k=1}^{K} v_{k} \frac{\mathbf{b}_{k}^{\mathrm{T}} \mathbf{p}_{3}}{\mathbf{p}_{3}^{\mathrm{T}} \mathbf{A}_{k} \mathbf{p}_{3}} \leq \xi \eta_{1}+(1-\xi) \eta_{2}$ and $\mathbf{1}^{\mathrm{T}} \mathbf{p}_{3} \geq$ $\xi \mathbf{1}^{\mathrm{T}} \mathbf{p}_{1}+(1-\xi) \mathbf{1}^{\mathrm{T}} \mathbf{p}_{2}$.

According to Lemma 1 , if we set $\mathbf{p}_{3}=\mathbf{p}_{\max }$, then

$$
\sum_{k=1}^{K} v_{k} \frac{\mathbf{b}_{k}^{\mathrm{T}} \mathbf{p}_{3}}{\mathbf{p}_{3}^{\mathrm{T}} \mathbf{A}_{k} \mathbf{p}_{3}} \leq \eta_{1} \text { and } \sum_{k=1}^{K} v_{k} \frac{\mathbf{b}_{k}^{\mathrm{T}} \mathbf{p}_{3}}{\mathbf{p}_{3}^{\mathrm{T}} \mathbf{A}_{k} \mathbf{p}_{3}} \leq \eta_{2}
$$

$$
\begin{aligned}
& \sum_{k=1}^{K} v_{k} \frac{\mathbf{b}_{k}^{\mathrm{T}} \mathbf{p}_{3}}{\mathbf{p}_{3}^{\mathrm{T}} \mathbf{A}_{k} \mathbf{p}_{3}}=\xi \sum_{k=1}^{K} v_{k} \frac{\mathbf{b}_{k}^{\mathrm{T}} \mathbf{p}_{3}}{\mathbf{p}_{3}^{\mathrm{T}} \mathbf{A}_{k} \mathbf{p}_{3}} \\
& \quad+(1-\xi) \sum_{k=1}^{K} v_{k} \frac{\mathbf{b}_{k}^{\mathrm{T}} \mathbf{p}_{3}}{\mathbf{p}_{3}^{\mathrm{T}} \mathbf{A}_{k} \mathbf{p}_{3}} \leq \xi \eta_{1}+(1-\xi) \eta_{2}, \\
& \mathbf{1}^{\mathrm{T}} \mathbf{p}_{3}=\xi \mathbf{1}^{\mathrm{T}} \mathbf{p}_{3}+(1-\xi) \mathbf{1}^{\mathrm{T}} \mathbf{p}_{3} \geq \xi \mathbf{1}^{\mathrm{T}} \mathbf{p}_{1}+(1-\xi) \mathbf{1}^{\mathrm{T}} \mathbf{p}_{2} .
\end{aligned}
$$

Therefore, $\mathbb{P} 1$ satisfies the time-sharing condition and the dual gap for our nonconvex problem is zero.

\section{REFERENCES}

[1] J. Li and P. Stoica, MIMO Radar Signal Processing. Hoboken, NJ, USA: Wiley, 2009.

[2] Y. Yu, A. P. Petropulu, and H. V. Poor, "Measurement matrix design for compressive sensing-based MIMO radar," IEEE Trans. Signal Process., vol. 59, no. 11, pp. 5338-5352, Nov. 2011.

[3] S. Gogineni and A. Nehorai, "Target estimation using sparse modeling for distributed MIMO radar," IEEE Trans. Signal Process., vol. 59, no. 11, pp. 5315-5325, Nov. 2011.

[4] Y. Yao, A. P. Petrppulu, and H. V. Poor, "CSSF MIMO radar: Compressivesensing and step-frequency based MIMO radar," IEEE Trans. Aerosp. Electron. Syst., vol. 48, no. 2, pp. 1490-1504, Apr. 2012.

[5] J. Xu, X.-Z. Dai, X.-G. Xia, L.-B. Wang, J. Yu, and Y.-N. Peng, "Optimization of multisite radar system with MIMO radars for target detection," IEEE Trans. Aerosp. Electron. Syst., vol. 47, no. 4, pp. 2329-2343, Oct. 2011.

[6] E. Fishler, A. Haimovich, R. S. Blum, L. J. Cimini, D. Chizhik, and R. A. Valenzuela, "Spatial diversity in radars-models and detection performance," IEEE Trans. Signal Process., vol. 54, no. 3, pp. 823-838, Mar. 2006.

[7] Q. He, N. H. Lehmann, R. S. Blum, and A. M. Haimovich, "MIMO-radar moving target detection in homogeneous clutter," IEEE Trans. Aerosp. Electron. Syst., vol. 46, no. 3, pp. 1290-1301, Jul. 2010.

[8] Q. He, R. S. Blum, H. Godrich, and A. M. Haimovich, "Cramer-Rao bound for target velocity estimation in MIMO radar with widely separated antennas," in Proc. 42nd Annu. Conf. Inf. Sci. Syst., Mar. 19-21, 2008, pp. 123-127.

[9] H. Godrich, A. M. Haimovich, and R. S. Blum, "Target localisation techniques and tools for multiple-input multiple-output radar," IET Radar, Sonar Navigat., vol. 3, no. 4, pp. 314-327, Aug. 2009.

[10] Q. He, R. S. Blum, H. Godrich, and A. M. Haimovich, "Target velocity estimation and antenna placement for MIMO radar with widely separated antennas," IEEE J. Sel. Topics Signal Process., vol. 4, no. 1, pp. 79-100, Feb. 2010.

[11] H. Godrich, A. M. Haimovich, and R. S. Blum, "Target localization accuracy gain in MIMO radar-based systems," IEEE Trans. Inf. Theory, vol. 56, no. 6, pp. 2783-2803, Jun. 2010.

[12] H. Godrich, A. P. Petropulu, and H. V. Poor, "Power allocation strategies for target localization in distributed multiple-radar architectures," IEEE Trans. Signal Process., vol. 59, no. 7, pp. 3226-3240, Jul. 2011.

[13] N. Garcia, A. M. Haimovich, M. Coulon, and M. Lops, "Resource allocation in MIMO radar with multiple targets for non-coherent localization," IEEE Trans. Signal Process., vol. 62, no. 10, pp. 2656-2666, May 2014.

[14] N. H. Lehmann et al., "Evaluation of transmit diversity in MIMO-radar direction finding," IEEE Trans. Signal Process., vol. 55, no. 5, pp. 22152225, May 2007.

[15] C. Wei, Q. He, and R. S. Blum, "Cramer-Rao bound for joint location and velocity estimation in multi-target non-coherent MIMO radars," in Proc. 44th Аnnu. Conf. Inf. Sci. Syst., Mar. 17-19, 2010, pp. 1-6.

[16] Q. He and R. S. Blum, "CramerRao bound for MIMO radar target localization with phase errors," IEEE Signal Process. Lett., vol. 17, no. 1, pp. 83-86, Jan. 2010.

[17] A. J. Conejo, E. Castillo, R. Minguez, and R. Garcia-Bertrand, Decomposition Techniques in Mathematical Programming: Engineering and Science Applications. Berlin, Germany: Springer-Verlag, 2006.

[18] S. Boyd, N. Parikh, E. Chu, B. Peleato, and J. Eckstein, "Distributed optimization and statistical learning via the alternating direction method of multipliers," Found. Trends Mach. Learn., vol. 3, no. 1, pp. 1-122, 2011.

[19] A. Simonetto and G. Leus, "Distributed maximum likelihood sensor network localization," IEEE Trans. Signal Process., vol. 62, no. 6, pp. 1424 1437, Mar. 2014.

[20] S. M. Kay, Fundamentals of Statistical Signal Processing, Volume II Detection Theory. Upper Saddle River, NJ, USA: Prentice-Hall, 1998.

[21] H. Godrich, A. M. Haimovich, and R. S. Blum, "Cramer Rao bound on target localization estimation in MIMO radar systems," in Proc. 42nd Annu. Conf. Inf. Sci. Syst., Mar. 19-21, 2008, pp. 134-139.

[22] H. Godrich, A. P. Petropulu, and H. V. Poor, "Sensor selection in distributed multiple-radar architectures for localization: A knapsack problem formulation," IEEE Trans. Signal Process., vol. 60, no. 1, pp. 247-260, Jan. 2012.

[23] F. Yousefian, A. Nedić, and U. V. Shanbhag, "On stochastic gradient an subgradient methods with adaptive steplength sequences," Automatica, vol. 48, no. 1, pp. 56-67, Jan. 2012.

[24] W. Yu and R. Lui, "Dual methods for nonconvex spectrum optimization of multicarrier systems," IEEE Trans. Commun., vol. 54, no. 7, pp. 13101322, Jul. 2006.

[25] S. Magnússon, P. C. Weeraddana, M. G. Rabbat, and C. Fischione, "On the convergence of alternating direction Lagrangian methods for nonconvex structured optimization problems," IEEE Trans. Control Netw. Syst., vol. PP, no. 99, pp. 1-14, doi: 10.1109/TCNS.2015.2476198, preprint.

[26] B. T. Polyak, Introduction to Optimization. New York, NY, USA: Optimization Software, Inc., 1987.

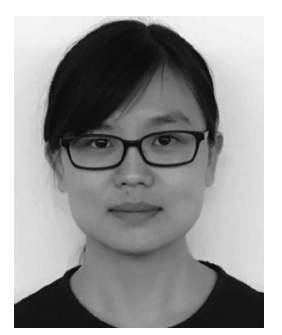

Ying Ma received the B.E. degree in electronic engineering from the Changchun Institute of Technology, Jin Lin, China, in 2011. She is currently working toward the Ph.D. degree with the Research Institute of Communication Technology, Beijing Institute of Technology, Beijing, China. Since July 2015, she has been with the Department of Electrical and Computer Engineering, University of Southampton, Southampton, U.K., where she is a visiting Ph.D. student under the supervision of Prof. L. Hanzo and Prof. S. Chen. Her research interests include distributed computation, multiple-input multiple-output systems, and cooperative communication. 


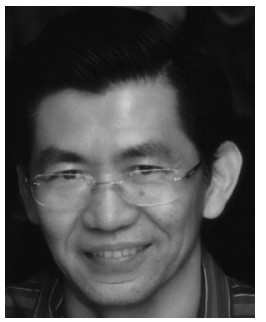

Sheng Chen (M'90-SM'97-F'08) received the B.Eng. degree in control engineering from the East China Petroleum Institute, Dongying, China, in January 1982, the Ph.D. degree in control engineering from the City University, London, U.K., in September 1986, and the D.Sc. degree from the University of Southampton, Southampton, U.K., in 2005. He held research and academic appointments with the University of Sheffield, the University of Edinburgh, and the University of Portsmouth, U.K., from 1986 to 1999 . Since 1999, he has been with the Electronics and Computer Science Department, University of Southampton, where he is a Professor of intelligent systems and signal processing. His research interests include adaptive signal processing, wireless communications, modeling and identification of nonlinear systems, neural network and machine learning, intelligent control system design, evolutionary computation methods, and optimization. He has authored more than 550 research papers. He is a Fellow of the United Kingdom Royal Academy of Engineering, a Fellow of the IET, and a Distinguished Adjunct Professor with King Abdulaziz University, Jeddah, Saudi Arabia. He was an ISI Highly Cited Researcher in engineering in 2004.

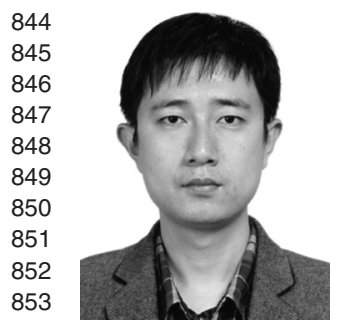

Chengwen Xing (S'08-M'10) received the B.Eng. degree from Xidian University, Xi' an, China, in 2005 , and the $\mathrm{Ph} . \mathrm{D}$. degree from the University of Hong Kong, Hong Kong, in 2010. Since September 2010, he has been with the School of Information and Electronics, Beijing Institute of Technology, Beijing, China, where he is currently an Associate Professor From September 2012 to December 2012, he was a Visiting Scholar at the University of Macau. His current research interests include statistical signal processing, convex optimization, multivariate statistics,

855 combinatorial optimization, massive MIMO systems, and high-frequency band 856 communication systems. He is currently serving as an Associate Editor for the 857 IEEE TRANSACTIONS ON VEHICULAR TECHNOLOGY, KSII Transactions on In858 ternet and Information Systems, Transactions on Emerging Telecommunications 859 Technologies, and China Communications.

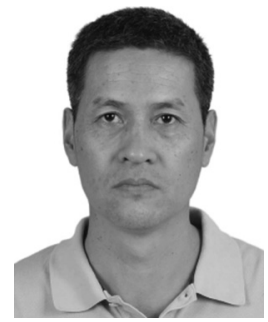

Xiangyuan Bu received the B.Eng., Master's, and 861 $\mathrm{Ph} . \mathrm{D}$. degrees from the Beijing Institute of Technol- 862 ogy, Beijing, China, in 1987, 1993, and 2007, re- 863 spectively. He is currently a Professor in the Wireless 864 Communications and Networks Laboratory, School 865 of Information and Electronics, Beijing Institute of 866 Technology. From July 1987 to September 1990, he 867 was a Researcher in the Institute of Harbin, 674 Fac- 868 tory, and from April 1993 to May 2002, he was a Re- 869 searcher in the No. 23 Institute of China Aerospace 870 Science and Industry. His current research interests 871 include wireless communication and signal processing.
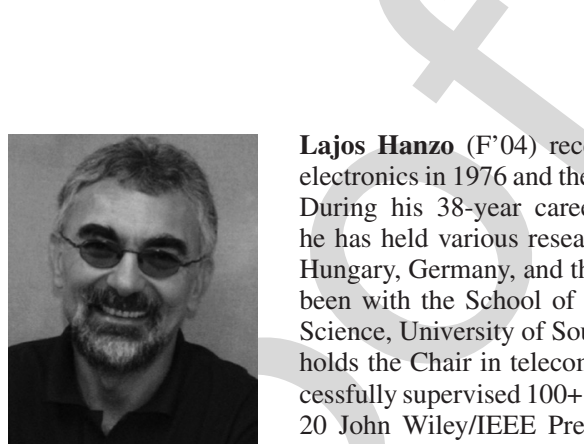

Lajos Hanzo (F'04) received the D.Sc. degree in 874 electronics in 1976 and the Doctorate degree in 1983. 875 During his 38-year career in telecommunications, 876 he has held various research and academic posts in 877 Hungary, Germany, and the U.K. Since 1986, he has 878 been with the School of Electronics and Computer 879 Science, University of Southampton, U.K., where he 880 holds the Chair in telecommunications. He has suc- 881 cessfully supervised $100+\mathrm{Ph}$. D. students, coauthored 882 20 John Wiley/IEEE Press books on mobile radio 883 communications, totalling in excess of 10000 pages, 884 published 1590 research entries at IEEE Xplore, acted both as the TPC Chair 885 and the General Chair of IEEE conferences, presented keynote lectures, and 886 has received a number of distinctions. He is currently directing a 60-strong 887 academic research team, working on a range of research projects in the field of 888 wireless multimedia communications sponsored by industry, the Engineering 889 and Physical Sciences Research Council, U.K., the European Research Coun- 890 cil's Advanced Fellow Grant, and the Royal Society's Wolfson Research Merit 891 Award. He is an enthusiastic supporter of industrial and academic liaison and he 892 offers a range of industrial courses. He is also a Governor of the IEEE VTS. In 893 2009, he received the honorary doctorate "Doctor Honoris Causa" by the Tech- 894 nical University of Budapest. During 2008-2012, he was the Editor-in-Chief 895 of the IEEE Press and also a Chaired Professor with Tsinghua University, Bei- 896 jing, China. His research is funded by the European Research Council's Senior 897 Research Fellow Grant. He is a Fellow of the Royal Academy of Engineering, 898 IET, and EURASIP. For further information on research in progress and asso- 899 ciated publications, please refer to http://www-mobile.ecs.soton.ac.uk. He has 900 $25000+$ citations. 873

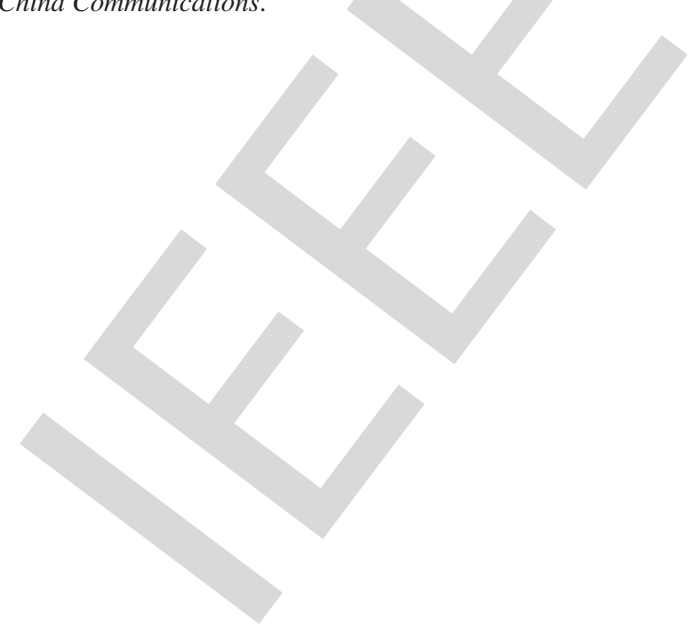


Q1. Author: "Scenario" is spelled as "senario." Please check.

Q2. Author: Please provide full bibliographic details in Ref. [25]. 